Aus der Abteilung Hämatologie und Onkologie

(Prof. Dr. med. L. Trümper)

im Zentrum Innere Medizin

der Medizinischen Fakultät der Universität Göttingen

\title{
Radioimmuntherapie \\ bei diffus großzelligen B-Zell-Lymphomen
}

Eine Auswertung des internationalen RIT-Registers

\author{
INAUGURAL-DISSERTATION \\ zur Erlangung des Doktorgrades \\ der Medizinischen Fakultät \\ der Georg August Universität zu Göttingen
}

vorgelegt von

Henrike Katharina Lankeit

aus

Köln

Göttingen 2012 
Dekan: $\quad$ Prof. Dr. med. C. Frömmel

I. Berichterstatter: Prof. Dr. med. L. Trümper

II. Berichterstatter: Prof. Dr. med. J. Meller

III. Berichterstatter: Prof. Dr. med. M. Oppermann

Tag der mündlichen Prüfung: 26.06.2012 


\section{Inhaltsverzeichnis}

$1 \quad$ Einleitung ............................................................................................................................................ 1

1.1 Diffus großzellige B-Zell-Lymphome ........................................................ 1

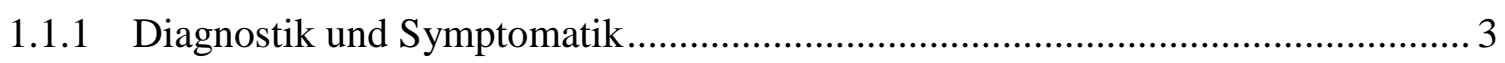

1.1.2 Stadieneinteilung und Risikostratifizierung ....................................................... 5

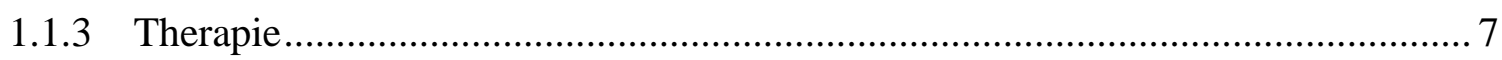

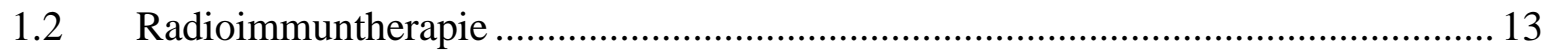

1.2.1 Bestandteile der Radioimmuntherapie ........................................................... 13

1.2.2 Klinische Anwendung der Radioimmuntherapie ............................................. 16

1.2.3 Radioimmuntherapie bei diffus großzelligen B-Zell-Lymphomen...................... 18

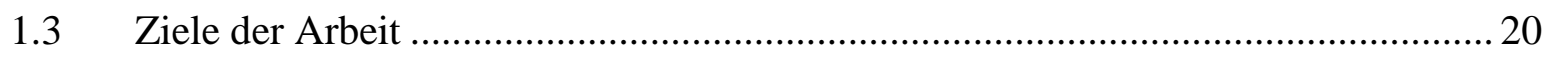

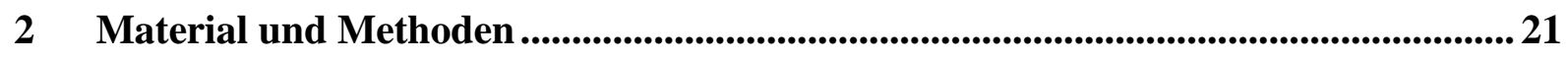

$2.1 \quad$ Internationales Radioimmuntherapie-Netzwerk ............................................. 21

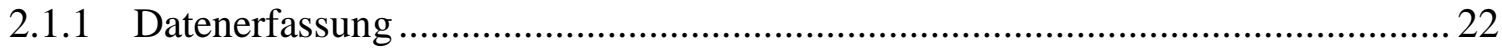

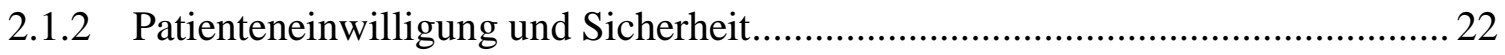

2.2 Patienten mit diffus großzelligem B-Zell-Lymphom ........................................ 23

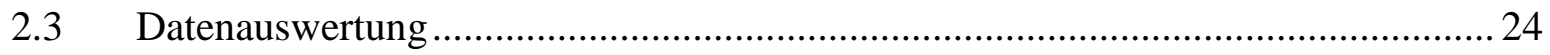

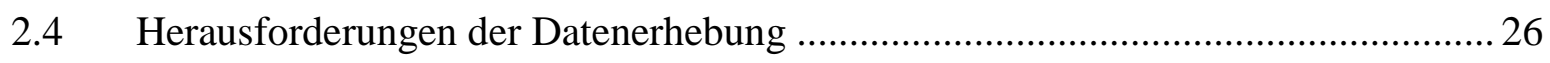

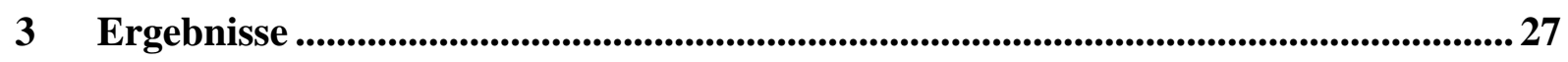

3.1 Charakteristika der Patienten und ihrer Erkrankung .......................................... 27

3.2 Indikationsstellung für die Radioimmuntherapie und ihre Anwendung ................. 32

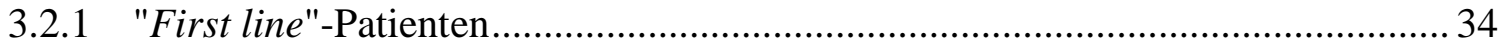

3.3 Klinisches Ergebnis nach Radioimmuntherapie ............................................... 39

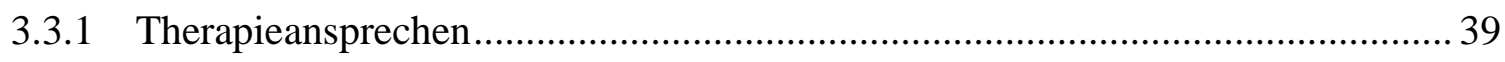

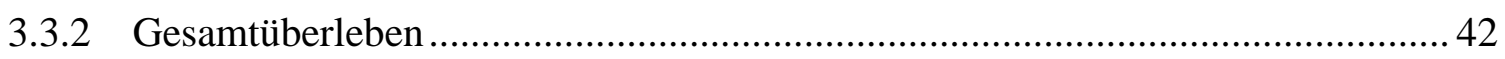

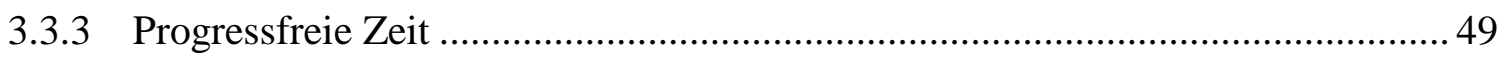

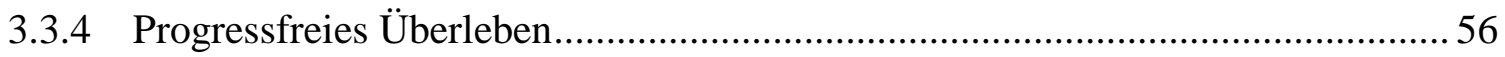

$3.4 \quad$ Unerwünschte Nebenwirkungen der Radioimmuntherapie ..................................58

3.4.1 Unerwünschte Arzneimittelwirkung bei "First line"-Patienten ............................ 61

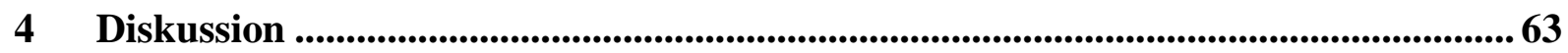

4.1 Die Bedeutung und Aussagekraft der untersuchten Registerdaten ......................... 63

4.1.1 Analyse und Diskussion der Charakteristika des Patientenkollektivs ..................64 64 
4.1.2 Analyse und Diskussion der klinischen Ergebnisse und unerwünschter Arzneimittelwirkungen. 66

4.2 Klinische Ergebnisse des RIT-Registers im Vergleich mit klinischen Studien zur Anwendung von RIT 70

4.2.1 Die Ergebnisse des Gesamtkollektivs im Vergleich mit den Ergebnissen zweier klinischer Studien .71

4.2.2 Die Ergebnisse des "First line"-Patientenkollektivs im Vergleich mit den Ergebnissen zweier klinischer Studien. .74

4.2.3 Unerwünschte Arzneimittelwirkungen der RIT bei Registerpatienten im

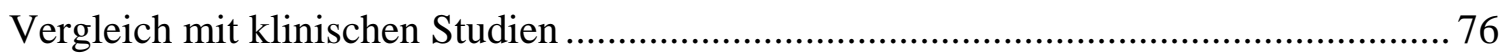

4.2.4 Bedeutung dieser Ergebnisse im wissenschaftlichen Kontext ............................ 79

4.3 Klinische Ergebnisse der RIT bei Registerpatienten im Vergleich zu den aktuellen Leitlinien der Therapie von DLBCL

4.3.1 Die Ergebnisse der RICOVER60-Studie und einer Studie der GELA im Vergleich mit den Ergebnissen der RIT-Registeranalyse . 82

4.3.2 Die Ergebnisse der MInT-Studie im Vergleich mit den Ergebnissen der RITRegisteranalyse. 85

4.3.3 Bedeutung dieser Ergebnisse im wissenschaftlichen Kontext ............................ 87

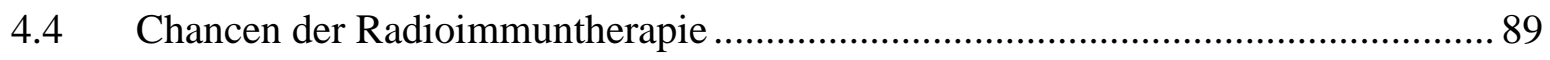

4.4.1 Offene Fragen in der Anwendung von RIT bei DLBCL .................................. 91

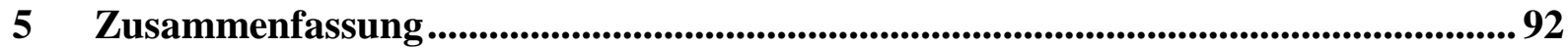

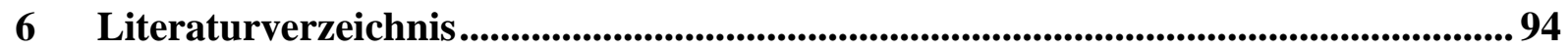

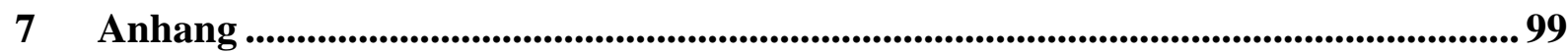

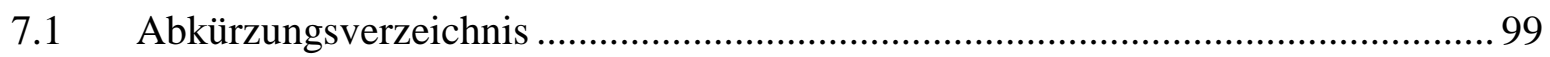

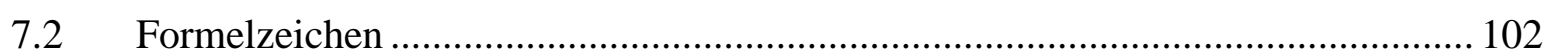

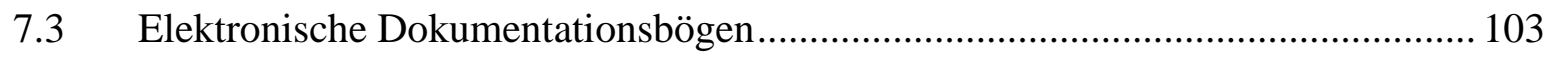




\section{Einleitung}

\subsection{Diffus großzellige B-Zell-Lymphome}

Diffus großzellige B-Zell-Lymphome (diffuse large B-cell lymphoma, DLBCL) sind als maligne Neoplasien des lymphatischen Systems der heterogenen Gruppe der Non-HodgkinLymphome (NHL) zugehörig. Eine einheitliche und allgemeingültige Einteilung der NHL, wie sie zumeist für andere Tumorentitäten üblich ist, bestand lange Zeit nicht und hat sich erst in der zweiten Hälfte des zwanzigsten Jahrhunderts stetig entwickelt. Erste Ansätze wurden durch die Kiel-Klassifikation (Lennert et al. 1975) im deutschsprachigen Raum realisiert, während sich im englischsprachigen Europa und den USA hauptsächlich die Working Formulation (Rosenberg et al. 1982) durchsetzte. 1994 wurde durch die Internationale Lymphom-Studiengruppe in transatlantischer Zusammenarbeit und unter Berücksichtigung des wissenschaftlichen Fortschritts die damals neue, international anerkannte, R.E.A.L.Klassifikation entwickelt, welche erstmals die unterschiedlichen Lymphomentitäten exakt definierte und nach klinischen, morphologischen sowie neueren immunologischen und genetischen Aspekten beschrieb (Harris et al. 1994). Zurzeit ist die vierte Auflage der WHO-Klassifikationen von 2008 (Jaffe 2009), welche sich aus der R.E.A.L.-Klassifikation entwickelte, das weitverbreitetste und allgemein genutzte Klassifikationssystem. In diesem überarbeiteten System werden NHL nach ihrer Zugehörigkeit zur B- oder T-Zell-Reihe, sowie dem jeweiligen Reifegrad der Ursprungszelle eingeteilt. Zusätzlich werden die verschiedenen Entitäten wie schon bei der R.E.A.L.-Klassifikation anhand ihrer Morphologie, Immunologie und Genetik unterschieden (siehe Tabelle 1.1). 


\section{Vorläufer B-Zell-Neoplasien}

- Vorläufer-B-lymphoblastische(s)-Leukämie / -Lymphom

\section{Reife B-Zell-Neoplasien}

- Chronisch lymphatische Leukämie, lymphozytisches Lymphom

- B-Zell-prolymphozytische Leukämie

- Lymphoplasmozytisches Lymphom

- Splenisches Marginalzonen-B-Zell-Lymphom

- Haarzellleukämie

- Plasmazellmyelom

- Extranodales Marginalzonen-B-Zell-Lymphom des MALT-Gewebes

- Nodales Marginalzonen-Lymphom

- Follikuläres Lymphom

- Mantelzelllymphom

- DLBCL

zentroblastisches, immunoblastisches und großzellig anaplastisches Lymphom primär kutane DLBCL, Borderline-Typen

- Burkitt-Lymphom / -Leukämie

\section{Vorläufer T-Zell-und NK-Zell-Neoplasien}

- Vorläufer-T-lymphoblastisches Lymphom, Blastisches NK-Zell-Lymphom

\section{Reife T-Zell- und NK-Zell-Neoplasien}

- T-Zell-prolymphozytische Leukämie

- T-Zell-großzellige granuläre lymphatische Leukämie

- Aggressive NK-Zell-Leukämie, chronische lymphoproliferative NK-Zell-Erkrankung

- Adulte(s) T-Zell-Leukämie / -Lymphom

- Extranodales NK- / T-Zell-Lymphom vom nasalen Typ

- Enteropathieassoziiertes Lymphom

- Hepatosplenisches Lymphom

- Subkutanes Pannikulitis-ähnliches T-Zell-Lymphom

- Mycosis fungoides, Sézary Syndrom

- Primär kutanes anaplastisches großzelliges Lymphom

- Peripheres T-Zell-Lymphom, unspezifiziert

- Angioimmunoblastisches T-Zell-Lymphom

- Anaplastisches großzelliges Lymphom

Tabelle 1.1: WHO-Klassifikation der NHL; modifiziert nach Jaffe (2009, S.524 und 526). 
Demnach zählen die DLBCL zu den aggressiven, hochmalignen NHL der reifen B-Zell-Reihe und können weiterhin in die Varianten zentroblastisches, immunoblastisches und großzelliges anaplastisches Lymphom unterteilt werden. Mit einem Anteil von 30,0-35,0 \% (Illidge und Tolan 2008; Morschhauser et al. 2007) und einer jährlichen Inzidenz von ungefähr 3-4/100.000 Einwohner in der Europäischen Union (Tilly und Dreyling 2010) stellen sie die weltweit häufigste Form der NHL dar. Ähnlich wie die meisten NHL liegt die Inzidenz der DLBCL bei Männern höher als bei Frauen, ferner zeigt sich ein mittleres Erkrankungsalter von 64 Jahren.

Immunphänotypisch zeichnen sich die Zellen der DLBCL durch die Expression der Oberflächenmarker CD20, CD79a, CD19 und CD22 aus (Tiemann und Trümper 2001). Besonders das Oberflächenantigen CD20 spielt im Rahmen der Immunchemotherapie, auf die im Folgenden noch genauer eingegangen wird, eine entscheidende Rolle.

\subsubsection{Diagnostik und Symptomatik}

Zur Diagnostik der DLBCL ist eine ausführliche Anamnese und klinische Untersuchung unerlässlich. Es lassen sich typische, jedoch wenig spezifische Symptome wie beispielsweise schmerzlose Lymphknotenvergrößerungen oder extranodale Raumforderungen, wie auch Spleno- und seltener Hepatomegalie, untersuchen. Allgemeinsymptome wie Müdigkeit, Blässe, Abgeschlagenheit und Infektneigung sind oft auf eine verstärkte Freisetzung von immunregulatorischen Zytokinen durch das maligne Lymphom zurückführen. Annähernd alle Patienten leiden zum Zeitpunkt der Diagnosestellung unter Fieber über $38{ }^{\circ} \mathrm{C}$, Nachtschweiß und ungewolltem Gewichtsverlust von mehr als 10,0\% des ursprünglichen Körpergewichts, welches man als B-Symptomatik zusammenfasst (Carbone et al. 1971).

Ein schneller Krankheitsbeginn und ein rasches Fortschreiten der Symptomatik sind kennzeichnend für DLBCL und erklären unter anderem ihre Zugehörigkeit zur Gruppe der aggressiven Lymphome, denen ein plötzliches Auftreten und ein schneller Verlauf zu eigen ist.

Weitere unverzichtbare diagnostische Maßnahmen nach der Anamnese und der klinischen Untersuchung sind klinisch-chemische Untersuchungen des Blutes und bildgebende Verfahren, welche mittels Computertomographie durchgeführt werden. Ebenso ist die Anfertigung einer Knochenmarkhistologie geboten. Die zur Diagnosestellung der DLBCL nötigen Untersuchungen sind zusammenfassend in Tabelle 1.2 dargestellt. 
- Anamnese und körperliche Untersuchung

- Nachweis von B-Symptomatik

- Laboruntersuchungen:

- Differenzialblutbild

- Bestimmung der Leberwerte, Laktatdehydrogenase (LDH), Harnsäure, Kalzium

- Serumelektrophorese

- Serum- $\beta_{2}$-Mikroglobulin

- Thorax-Röntgenaufnahme

- CT von Abdomen, Becken und meistens des Thorax

- Knochenmarkbiopsie

- Lumbalpunktion bei positiver Knochenmarkbiopsie

Tabelle 1.2: Klinische Untersuchungen zur Diagnosestellung von DLBCL.

Zur endgültigen Verifizierung der Diagnose und Einteilung nach der WHO-Klassifikation ist eine histologische, immunhistochemische und molekulargenetische Untersuchung eines exzidierten, befallenen Lymphknotens beziehungsweise einer extranodalen Gewebeprobe unbedingt erforderlich (Tilly und Dreyling 2010). 


\subsubsection{Stadieneinteilung und Risikostratifizierung}

Nach der Diagnosestellung der DLBCL sind eine klinische Stadieneinteilung sowie Risikostratifizierung des Patienten vonnöten, um dessen Therapie entsprechend planen und festlegen zu können. Durch eine einheitliche Einteilung der Patienten wird das Verständnis und die Kommunikation innerhalb verschiedener Zentren und zwischen den behandelnden Ärzten verbessert und erleichtert.

Die klinische Stadieneinteilung aggressiver Lymphome erfolgt mittels der ursprünglich für Hodgkin-Lymphome entwickelten Ann-Arbor-Klassifikation in die Stadien I bis IV:

I Beteiligung einer einzigen Lymphknotenregion oder extranodaler Struktur

II Beteiligung von zwei oder mehrerer Lymphknotenregionen derselben Zwerchfellseite oder einer extranodalen Struktur und einer oder mehrerer Lymphknotenregionen derselben Zwerchfellseite

III Beteiligung von Lymphknotenregionen oder extranodaler Strukturen auf beiden Seiten des Zwerchfells

IV Diffuser oder disseminierter Befall von extralymphatischem Gewebe mit oder ohne Lymphknotenbeteiligung.

Durch verschiedene Zusätze kann die Diagnose noch genauer wiedergegeben werden. Somit steht der Zusatz A für das Fehlen und der Zusatz B für das Vorhandensein einer B-Symptomatik. Als weitere Beispiele steht E für den Befall extranodaler Strukturen, S für den Befall der Milz und M für den Befall des Knochenmarks (Carbone et al. 1971).

Der Patientenzustand wird außerdem mit Hilfe des Internationalen Prognostischen Index (IPI) beschrieben, welcher dazu dienen soll, die Prognose des Patienten abzuleiten und anhand deren die Behandlungsstrategien auszurichten. Der 1993 mittels einer retrospektiven Analyse der Krankheitsverläufe von 2.031 NHL-Patienten entwickelte IPI nutzt fünf Risikofaktoren, um Patienten mit aggressiven Lymphomen in die vier Gruppen 'niedriges', 'niedrigintermediäres', 'intermediär hohes' und 'hohes Risiko' einzuteilen. Im klinischen Gebrauch werden Patienten mit einem niedrigen bis niedrigintermediärem Risiko (IPI =0-2) häufig als Niedrigrisikopatienten und solche mit einem IPI > 2 (intermediär hohes bis hohes Risiko) als Hochrisikopatienten zusammengefasst. 
Bei den fünf Risikofaktoren handelt es sich um:

- $\quad$ Alter $\geq 60$ Jahre

- Tumorausbreitung nach Ann-Arbor im Stadium III oder IV

- Befall von mehr als einer extranodalen Struktur

- $\quad$ schlechter Allgemeinzustand (Karnofsky-Index $\leq 70$ oder ECOG-Punktzahl $\geq 2$ )

- erhöhter Serumspiegel der Laktatdehydrogenase (LDH).

Je nach Anzahl der zutreffenden Parameter kann jeder Patient mit einem NHL einer der benannten Risikogruppen zugeordnet werden und somit dessen Überlebenswahrscheinlichkeit ermittelt und der Therapieerfolg abgeschätzt werden (siehe Tabelle 1.3).

\begin{tabular}{|c|c|c|c|}
\hline Risikogruppe & $\begin{array}{c}\text { Anzahl der } \\
\text { Risikofaktoren }\end{array}$ & $\begin{array}{c}\text { 2-Jahres- } \\
\text { Überlebensrate } \\
{[\%]}\end{array}$ & $\begin{array}{c}\text { 5-Jahres- } \\
\text { Überlebensrate } \\
\text { [\%] }\end{array}$ \\
\hline Niedriges Risiko & $0-1$ & 84,0 & 73,0 \\
\hline $\begin{array}{c}\text { Niedrigintermediäres } \\
\text { Risiko }\end{array}$ & 2 & 66,0 & 51,0 \\
\hline $\begin{array}{c}\text { Intermediär hohes } \\
\text { Risiko }\end{array}$ & 3 & 54,0 & 43,0 \\
\hline Hohes Risiko & $4-5$ & 34,0 & 26,0 \\
\hline
\end{tabular}

Tabelle 1.3: Internationaler Prognostischer Index für Non-Hodgkin-Lymphome; modifiziert nach Shipp et al. (1993, S.992).

Ein vereinfachtes Modell, welches nur die Parameter 'Tumor-Stadium', 'Serum-LDH' und 'Allgemeinzustand' des Patienten miteinschließt, wurde für eine jüngere Patientengruppe, d.h. < 60 Jahre, entwickelt. Dieser, in Tabelle 1.4 dargestellte, altersadaptierte IPI (aaIPI) berücksichtigt nicht den extranodalen Befall, welcher bei diesem Patientenkollektiv keinen unabhängigen Risikofaktor darstellt (Shipp et al. 1993). 


\begin{tabular}{|c|c|c|c|}
\hline Risikogruppe & $\begin{array}{c}\text { Anzahl der } \\
\text { Risikofaktoren }\end{array}$ & $\begin{array}{c}\text { 2-Jahres- } \\
\text { Überlebensrate } \\
{[\%]}\end{array}$ & $\begin{array}{c}\text { 5-Jahres- } \\
\text { Überlebensrate } \\
\text { [\%] }\end{array}$ \\
\hline $\begin{array}{c}\text { Niedriges Risiko } \\
\text { Niedrigintermediäres } \\
\text { Risiko }\end{array}$ & 1 & 90,0 & 83,0 \\
\hline $\begin{array}{c}\text { Intermediär hohes } \\
\text { Risiko }\end{array}$ & 2 & 79,0 & 69,0 \\
\hline Hohes Risiko & 3 & 59,0 & 46,0 \\
\hline
\end{tabular}

Tabelle 1.4: Altersadaptierter Internationaler Prognostischer Index für Non-HodgkinLymphome; modifiziert nach Shipp et al. (1993, S.992).

\subsubsection{Therapie}

Hochmaligne Lymphome, wie die DLBCL, weisen ein aggressives Wachstum mit einer hohen Zellteilungsrate auf und zeigen somit ein gutes Ansprechen auf Chemo- und Strahlentherapien, welche sich besonders gegen schnell teilende Zellen richten. Es kann somit auch in fortgeschrittenen Stadien eine dauerhafte Remission und Heilung der malignen Lymphome erreicht werden. Aus diesem Grund besteht bei der Therapie der DLBCL stets ein kurativer Ansatz mit intensiver Behandlungsstrategie unabhängig vom Krankheitsstadium. Dies gilt auch für ältere Patienten, obwohl bei diesen die Heilungschancen geringer sind und Rezidive gehäuft auftreten können. Dennoch ist nur in Ausnahmefällen ein palliativer Therapieansatz gerechtfertigt (Zinzani et al. 2008).

Trotz der hohen Strahlenempfindlichkeit der DLBCL ist der Stellenwert einer Strahlentherapie nicht standardisiert. Eine alleinige Bestrahlung ist heutzutage nicht mehr von Bedeutung, da diese besonders für ältere Patienten, selbst im frühen und lokalisierten Stadium der Erkrankung, einer Chemotherapie in Kombination mit Strahlentherapie unterlegen ist (Spicer et al. 2004). In Folge dieser Erkenntnis galt eine kombinierte Radiochemotherapie als Leitlinie der DLBCL-Behandlung, bis von der französischen Studiengruppe für Lymphome bei Erwachsenen (Groupe d'Etude des Lymphomes d'Adulte, GELA) gezeigt werden konnte, dass eine alleinige, intensive Polychemotherapie bessere Ergebnisse in Bezug auf das Gesamtüberleben der Patienten erzielt (Reyes et al. 2005). Somit ist eine alleinige 
Polychemotherapie mit voller Zyklusanzahl der kombinierten Radiochemotherapie, bei der zumeist die Chemotherapiezyklen reduziert werden, vorzuziehen. Eine weitere Option, Bestrahlung einsetzen zu können, stellt die konsolidierende Strahlentherapie nach vollständiger Chemotherapie dar. Unterstützt wird dieser Therapieansatz durch die Ergebnisse einer kleinen Studie aus Mexiko, die nachweisen konnte, dass Strahlentherapie als Konsolidierung eine effektive Maßnahme für DLBCL-Patienten mit hohem IPI darstellt sowie ihr Gesamtüberleben und ereignisfreies Überleben verlängert (Aviles et al. 2004). Des Weiteren beweist eine Studie der European Organisation for Research and Treatment of Cancer (EORTC) eine vergleichbare Anwendbarkeit von Strahlentherapie und Hochdosischemotherapie mit autologer Stammzelltransplantation als Konsolidierung nach ErstlinienChemotherapie (Moser et al. 2006). Dennoch ist die Rolle der konsolidierenden Strahlentherapie nicht klar definiert oder außerhalb von prospektiven Studien zu empfehlen, da die beiden oben genannten Studien jeweils nur eine sehr kleine Patientenanzahl untersuchten und somit nur eine geringe wissenschaftliche Evidenz aufweisen (Illidge und Tolan 2008).

Auch in folgenden, jedoch größeren Studien, welche insbesondere die Anwendung des im weiteren Verlauf beschriebenen Antikörpers Rituximab untersuchen, zeigt sich lediglich ein marginaler Effekt der konsolidierenden Strahlentherapie bei DLBCL. Hier wurden jeweils Patienten mit anfänglich großer Tumorlast oder extranodalem Befall zusätzlich zu einer Polychemotherapie mit oder ohne Rituximab bestrahlt, wobei jedoch kein eindeutiger Einfluss auf den Therapieerfolg nachgewiesen werden konnte (Pfreundschuh et al. 2006; Pfreundschuh et al. 2008a; Pfreundschuh et al. 2008b). Somit wird aktuell die Relevanz der konsolidierenden Strahlentherapie bei Patienten mit DLBCL in der laufenden UNFOLDERStudie der Deutschen Studiengruppe Hochmaligne Non-Hodgkin-Lymphome (DSHNHL) untersucht (Pfreundschuh et al. 2011).

Im Gegensatz zur Strahlentherapie ist die Bedeutung der Chemotherapie in der Behandlung von DLBCL eindeutig festgelegt. Als Therapiestandard gilt das 1976 eingeführte CHOPSchema, eine Polychemotherapie bestehend aus den Zytostatika Cyclophosphamid, Doxorubicin (Hydroxyldaunorubicin) und Vincristin (Oncovin®) sowie dem Steroid Prednison, welches für zwei Drittel der Patienten eine komplette Remission (complete remission, CR) sowie für immerhin rund ein Drittel der Patienten im fortgeschrittenen Stadium Heilung erbrachte und darüber hinaus im Vergleich mit anderen Polychemotherapien eine geringere Toxizität aufwies (Fisher et al. 1993). 
Trotz dieser guten Ergebnisse blieb immer noch ein großer Anteil an Patienten, welche nicht auf die CHOP-Chemotherapie ansprachen oder nach kurzer Zeit Rezidive aufwiesen. Diese nicht zufriedenstellenden Untersuchungsergebnisse regten viele Arbeitsgruppen an, das CHOP-Schema $\mathrm{zu}$ modifizieren, weiter $\mathrm{zu}$ entwickeln und mit unterschiedlichen Therapieansätzen zu kombinieren, um bessere Ansprechraten zu erzielen beziehungsweise die Anzahl an Rezidiven zu senken. In Anlehnung an den IPI werden die Patienten dabei in drei verschiedene Gruppen unterteilt, die sich jeweils in ihrer optimalen Therapiestrategie unterscheiden sollen. Es handelt sich dabei um Patienten, die jünger als 60 Jahre mit einem niedrigen Risikoindex (IPI < 2), solche, die jünger als 60 Jahre mit einem hohem Risikoindex (IPI $\geq 2$ ), und schließlich um Patienten, die älter als 60 Jahre sind. Im Folgenden soll nun kurz auf einige der unterschiedlichen Entwicklungen der DLBCL-Therapie innerhalb der jeweiligen Therapiegruppen eingegangen werden.

Pfreundschuh et al. konnten beispielsweise zeigen, dass eine Intervallverkürzung und die damit einhergehende Dosisintensivierung des bis dahin gebräuchlichen drei-wöchentlichen Rhythmus des CHOP-Schemas (CHOP-21) auf einen zwei-wöchentlichen Rhythmus (CHOP-14) in der Gruppe der über 60-Jährigen zu höheren CR-Raten und besseren Langzeitergebnissen führte (Pfreundschuh et al. 2004a). Die Ergänzung von Etoposid als weiteres Zytostatikum zum CHOP-Schema (CHOEP) führte in diesem Patientenkollektiv ebenfalls zu einem größeren Therapieerfolg, jedoch nicht so eindrücklich, wie dies bei der Gruppe der jüngeren Patienten mit niedrigem Risiko und guter Prognose der Fall war. Bei dieser Patientengruppe führte die Etoposidzugabe nämlich zu einem signifikanten Überlebensvorteil (Pfreundschuh et al. 2004b). Die Überlegenheit von CHOEP-21 über CHOP-21 bei dieser Patientengruppe konnte später auch von der MInT-Studiengruppe (MabThera International Trial) bestätigt werden (Pfreundschuh et al. 2006).

Besonders schwierig gestaltete sich die Suche nach einer adäquaten Therapiestrategie für die Gruppe der jüngeren Hochrisikopatienten:

In der Hoffnung auf verbesserte Heilungsraten und Überlebenskurven wurden unter anderem verschiedene Protokolle von Hochdosischemotherapie (HDC) in Kombination mit Stammzelltransplantation von unterschiedlichen Studiengruppen untersucht, woraus jedoch sehr heterogene Ergebnisse hervorgingen. Eine Studie der GELA beispielsweise, welche eine Konsolidierung nach Polychemotherapie mittels sequentieller Chemotherapie mit einer HDC gefolgt von autologer Stammzelltransplantation (autoSZT) vergleicht, zeigte einen Überlebensvorteil für Patienten mit höherem Risikoindex, welche mit konsolidierender HDC 
behandelt wurden (Haioun et al. 2000). Andere Studien dagegen konnten keinen signifikanten Unterschied zwischen einer HDC mit autoSZT und dem konventionellen Polychemotherapieprotokoll CHOP demonstrieren. Ein Beispiel hierfür ist eine Studie der DSHNHL aus dem Jahr 2002 (Kaiser et al. 2002). Des Weiteren publizierte die GELA eine Studie, in der eine frühe HDC mit autoSZT einer konventionellen Polychemotherapie sogar unterlegen war, so dass sich eine Hochdosischemotherapie nicht als neuer Therapiestandard durchsetzten konnte (Gisselbrecht et al. 2002). Infolgedessen untersuchte die DSHNHL ein dosisgesteigertes und intensiviertes Protokoll der CHOEP-Chemotherapie mit nachfolgender autoSZT (MegaCHOEP) als weitere mögliche Therapieoption. Es zeigte sich hierbei, dass besonders eine frühzeitige Steigerung der verabreichten Dosis ausschlaggebend für die Therapie junger Hochrisikopatienten ist (Schmitz et al. 2006). In folgenden Studien wurde jedoch demonstriert, dass das MegaCHOEP-Schema bei dieser Patientengruppe dem, im nächsten Kapitel beschriebenem, konventionellen Chemotherapieprotokoll in Kombination mit dem Antikörper Rituximab unterlegen ist und somit aktuell nicht mehr empfohlen werden kann (Held et al. 2011; Schmitz et al. 2011).

Bei der Behandlung rezidivierter DLBCL nimmt die HDC mit Stammzelltransplantation dagegen einen hohen Stellenwert ein. So konnte die sogenannte PARMA-Studie die Überlegenheit einer HDC und anschließender autoSZT gegenüber der üblichen Polychemotherapie bei rezidivierten, chemotherapiesensiblen, aggressiven Lymphomen beweisen (Philip et al. 1995). Eine HDC in Kombination mit autoSZT oder jedoch eine allogene Stammzelltransplantation (alloSZT) mit weniger intensiver Konditionierung scheinen sogar die einzigen kurativen Therapieansätze für rezidivierte aggressive NHL zu sein. Da immerhin 15-40 \% der mit Therapiestandard behandelten DLBCL-Patienten ein Rezidiv erleiden, hat sich diese Zweitlinientherapie nunmehr fest etabliert, obwohl nicht alle Patienten für solch eine intensive Therapie geeignet sind. Für ältere Patienten könnte die Toxizität dieser Kombinationstherapie beispielsweise zu groß sein. Außerdem ist das Risiko für Zweittumore und Rezidive relativ hoch und der therapeutische Erfolg bei chemotherapierefraktären, mehrfach und insbesondere mit dem im Folgenden beschriebenen Antikörper Rituximab vorbehandelten sowie innerhalb des ersten Jahres oder im Verlauf mehrfach rezidivierten Lymphomen relativ gering (Gisselbrecht et al. 2009; Gisselbrecht et al. 2010). Des Weiteren ist zur Zeit noch nicht endgültig geklärt, welches HDC-Protokoll bei rezidivierten oder therapierefraktären Lymphomen den größten und sichersten Therapieerfolg bringt. Kürzlich veröffentlichte Untersuchungen zeigen jedoch, dass das HDC-Schema R-DHAP, bestehend aus dem Antikörper Rituximab (vergleiche 1.1.3.1) und den Zytostatika 
Dexamethason, Cytarabin (Ara C) in hoher Dosis sowie Cisplatin, in Kombination mit einer autoSZT bei einer bestimmten Unterart der DLBCL anderen Therapieprotokollen überlegen ist. Diese Ergebnisse sollen nun in zukünftigen Studien genauer untersucht werden (Thieblemont et al. 2011).

\subsubsection{Rituximab}

Die Entwicklung des chimären monoklonalen anti-CD20-Antikörpers Rituximab bewirkte einen großen Fortschritt in der Behandlung von malignen Lymphomen und konnte sich auch als Therapeutikum gegen DLBCL etablieren. Voraussetzung für die Wirksamkeit von Rituximab ist eine möglichst zahlreiche Expression des Oberflächenmarkers CD20 auf den entarteten Zellen des malignen Lymphoms, an denen der Antikörper anbinden kann. Diese Bindung bewirkt sowohl die komplement- als auch antikörperabhängige Zytotoxizität Rituximabs. Weiterhin induziert der Antikörper über einen direkten Eingriff in die zelluläre Homöostase die Apoptose der betreffenden Zelle (Chapuy et al. 2006). Nachdem die Immuntherapie bereits erfolgreich in der Therapie indolenter Lymphome eingesetzt wurde, konnte eine Studie der GELA auch ein effektives Ansprechen der DLBCL auf Rituximab nachweisen. In dieser Studie wurde das bis dahin übliche CHOP-Schema mit CHOP plus Rituximab (R-CHOP) bei älteren Patienten (60 bis 80 Jahre) verglichen und ergab signifikant höhere Remissionsraten sowie ein verlängertes ereignisfreies und Gesamtüberleben für die Patientengruppe, die das R-CHOP-Schema erhalten hatte (Coiffier et al. 2002). Eine kürzlich veröffentlichte Langzeituntersuchung ergab, dass auch nach einer 10-Jahres-Verlaufskontrolle die ursprünglich erreichten Ergebnisse immer noch Bestand haben (Coiffier et al. 2010). Die DSHNHL untersuchte daraufhin in der RICOVER60-Studie die optimale Zyklusanzahl des RCHOP-Schemas in dem Patientenkollektiv über 60 Jahre und erzielte mit sechs Zyklen CHOP-14 und achtmaliger Applikation von Rituximab (6 x CHOP-14 + 8 x R-14) die besten Ergebnisse (Pfreundschuh et al. 2008b).

Die Kombination von Rituximab und CHOP stellt auch in der Patientengruppe unter 60 Jahren mit niedrigem Risikoprofil, wie von der MInT-Studiengruppe untersucht wurde, eine effektivere Therapieoption als eine alleinige Polychemotherapie dar, so dass auch hier Immuntherapie mit Rituximab standardisiert eingesetzt werden kann. Der zuvor beschriebene Vorteil des nebenwirkungsreicheren CHOEP-Protokolls lässt sich in Kombination mit Rituximab (R-CHOEP) dagegen nicht mehr nachweisen (Pfreundschuh et al. 2006; Pfreundschuh et al. 2011). 
Die DSHNHL konnte zudem kürzlich zeigen, dass die Addition von Rituximab auch zum dosisgesteigerten und intensivierten Chemotherapie-Konzept MegaCHOEP, welches bei Hochrisikopatienten unter 60 Jahren in Studien der DSHNHL eingesetzt wird, eine signifikante Verbesserung der Ergebnisse verglichen mit MegaCHOEP ohne Rituximab erzielt. Auch hierbei bewirkt der Zusatz des monoklonalen Antikörpers eine Verlängerung des ereignisfreien und des Gesamtüberlebens (Glass et al. 2010). Da R-MegaCHOEP sich jedoch im Vergleich zur konventionellen CHOEP-Therapie, welche mit Rituximab kombiniert appliziert wurde, als signifikant unterlegen zeigt, kann dieses Protokoll nicht mehr empfohlen werden (Held et al. 2011). Die 2011 auf dem jährlichen Treffen der Amerikanischen Gesellschaft für Klinische Onkologie (American Society of Clinical Oncology, ASCO) präsentierten, endgültigen Ergebnisse der MegaCHOEP-Studie der DSHNHL zeigen sogar, dass in der Gruppe der jungen Hochrisikopatienten die bis dato besten Therapieergebnisse mit einer konventionellen Chemotherapie in Kombination mit Rituximab, nämlich acht Zyklen R-CHOEP und sechs Zyklen Rituximab (8 x CHOEP-14 + 6 x R-14), erreicht werden (Schmitz et al. 2011).

Die Hinzunahme von Rituximab hat in allen drei Patientengruppen eine deutliche Steigerung des Therapieerfolgs bei geringem Nebenwirkungsprofil zur Folge und ist demgemäß ein fest etablierter Teil der Therapie der DLBCL in den aktuellen Leitlinien geworden. In diesen wird die Anwendung von sechs Zyklen CHOP-14 kombiniert mit acht Zyklen Rituximab für Patienten von mindestens 60 Jahren und sechs Zyklen R-CHOP-21 für Niedrigrisikopatienten als Standard empfohlen. Jüngere Hochrisikopatienten zeigen den größten Therapieerfolg bei acht Zyklen R-CHOEP-14, wobei jedoch noch kein ausreichend effizienter Therapiestandard besteht und häufig in Anlehnung an die beiden anderen Patientengruppen sechs bis acht Zyklen R-CHOP-21 verabreicht werden (Held et al. 2011; Tilly und Dreyling 2010). Es bleiben besonders die Behandlungsmöglichkeiten der Patienten mit hohem IPI unbefriedigend und erfordern noch effektivere und innovative Behandlungsmodelle (Morschhauser et al. 2007). Des Weiteren stellen rezidivierte oder therapierefraktäre Lymphome ein beständiges klinisches Behandlungsproblem dar.

Durch die Markierung eines Antikörpers mit Radiokonjugaten soll eine Verbindung der kombinierten Immun-Chemotherapie mit strahlentherapeutischen Strategien geschaffen werden, auf welche im folgenden Kapitel näher eingegangen wird. 


\subsection{Radioimmuntherapie}

Die Radioimmuntherapie (RIT) stellt als kombinierte, systemische Immun- und Strahlentherapie eine neue, effektive Strategie in der Behandlung maligner Erkrankungen dar. Die von Radioisotopen ausgehende Radioaktivität wird mittels Antikörper, welche an tumorassoziierte Antigene binden, selektiv auf die Tumorzellen gerichtet. Dadurch wird eine Reduktion der strahleninduzierten Nebenwirkungen erreicht, denn Gewebe, welche nicht unmittelbar dem Tumorgewebe anliegen oder das entsprechende Antigen nicht exprimieren, werden weitestgehend von der Radioaktivität verschont. Ferner wird auch durch die direkte Antikörperbindung eine zytotoxische Wirkung erreicht, welche das Tumorgewebe zusätzlich schwächt (Emmanouilides 2007).

Die RIT führte schon Anfang der neunziger Jahre zu ersten Erfolgen in der Therapie von NHL (Kaminski et al. 1993; Press et al. 1993). Seither arbeiten unterschiedliche Studiengruppen stetig weiter an der Entwicklung der RIT bei NHL, einschließlich der DLBCL, sodass inzwischen zwei Radioimmunkonjugate zur Therapie maligner Lymphome zugelassen wurden. Dabei handelt es sich einerseits um ${ }^{90}$ Yttrium-Ibritumomab Tiuxetan $\left({ }^{90} \mathrm{Y}-\mathrm{IT}\right.$; Handelsname: Zevalin $\left.{ }^{\circledR}\right)$ sowie um ${ }^{131}$ Iod-Tositumomab ( ${ }^{131} \mathrm{I}-\mathrm{T}$; Handelsname: Bexxar®) andererseits, auf deren Aufbau, Wirkungsweise und Therapieerfolg in den folgenden Kapiteln näher eingegangen werden soll.

\subsubsection{Bestandteile der Radioimmuntherapie}

Bei der RIT handelt es sich um eine Kombinationstherapie, deren Wirkprinzip aus der Zusammenarbeit verschiedener Elemente resultiert.

Neben seiner eigenen direkten Zytotoxizität fungiert der Antikörper als Transporter des Radionuklids zur Tumorzelle. Dabei soll der Antikörper möglichst selektiv an ein tumorassoziiertes Antigen, welches bestenfalls in hohen Maßen auf den Tumorzellen exprimiert wird, binden (Emmanouilides 2007). Die zur Therapie der DLBCL und anderer NHL verwendeten murinen Antikörper binden an den Oberflächenmarker CD20, der auf über $90 \%$ der malignen B-Zell-Lymphome und auf reifen, gesunden B-Zellen, nicht jedoch auf ihren Vorstufen oder Plasmazellen, vorkommt (Stashenko et al. 1980). Dieses Antigen wird außerdem weder von der Zelloberfläche abgestoßen, sodass es frei im Blut zirkulieren würde, 
noch in die Zelle internalisiert und stellt somit als beständiges Zelloberflächenprotein ein ideales Ziel für die RIT dar (Chapuy et al. 2007).

Wie in Abbildung 1.1 dargestellt, wird zur Bildung von ${ }^{90} \mathrm{Y}$-IT der monoklonale Antikörper Ibritumomab mittels des Chelators Tiuxetan fest mit dem Radionuklid ${ }^{90}$ Yttrium $\left({ }^{90} \mathrm{Y}\right)$ verbunden, während bei ${ }^{131} \mathrm{I}-\mathrm{T}$ eine direkte kovalente Bindung zwischen dem Antikörper Tiuxetan und dem Radionuklid ${ }^{131} \operatorname{Iod}\left({ }^{131} \mathrm{I}\right)$ besteht.

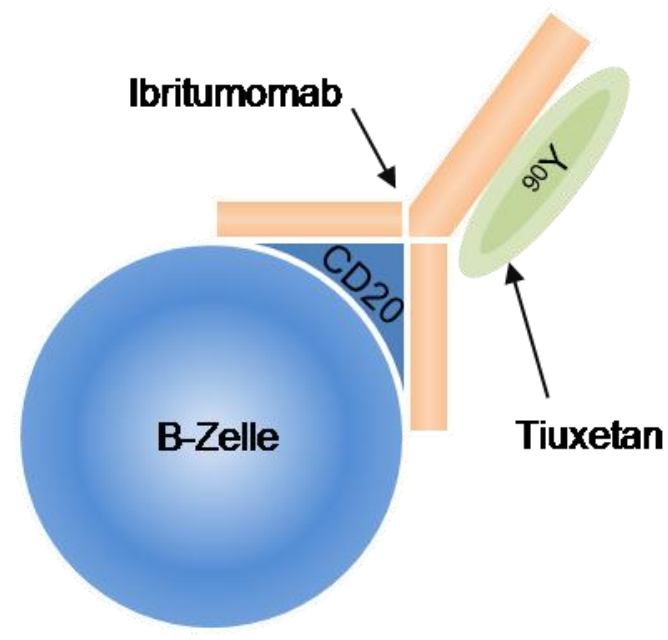

Abbildung 1.1: Bindung von ${ }^{90} \mathrm{Y}$-IT an den Oberflächenmarker CD20; modifiziert nach Gregory et al. (2009, S.6).

Die RIT nutzt die Strahlensensibilität der B-Zell-Lymphome effizient aus, indem instabile Atome, die Radionuklide, in direkte Nähe mit der zu bestrahlenden Tumormasse gebracht werden. Als Strahlungsquelle eignen sich dabei Isotope, die eine möglichst hohe Energie auf kurzer Distanz abgeben, sowie Nuklide mit einer relativ kurzen Halbwertszeit, um strahleninduzierte Spätfolgen zu limitieren und solche mit geringer Affinität zur Anreicherung in bestimmten Geweben. Ungeeignet zur RIT scheinen $\alpha$-Emitter zu sein, da durch ihre geringe Gewebedurchdringung der weiter unten erklärte "Kreuzfeuereffekt" nur eingeschränkt zu Stande kommen kann. Hat ein Radionuklid einen hohen Anteil an besonders durchdringender $\gamma$-Strahlung, wie es bei ${ }^{131} \mathrm{I}$ der Fall ist, führt dies zu einer relativ hohen Strahlenbelastung des gesamten Körpers sowie der Umgebung, während dem Tumor selber eine vergleichsweise geringere Dosis zugeführt wird. Somit sind reine $\gamma$-Emitter für die RIT ebenfalls nicht uneingeschränkt zu empfehlen. Dagegen sind hoch energetische $\beta$-Strahler, 
wie ${ }^{90} \mathrm{Y}$, durch ihre längere Reichweite besonders zur Behandlung größerer, schlecht erreichbarer Tumormassen geeignet (Emmanouilides 2007).

Es besteht die Möglichkeit, dass maligne Zellen, die auf Grund mangelnder CD20-Expression oder schlechter Vaskularisierung des Tumors dem Antikörper nicht direkt zugänglich sind, überleben und später zu Rezidiven führen. Durch die höhere Strahlenenergie von ${ }^{90} \mathrm{Y}$ überwinden die abgestrahlten Elektronen größere Distanzen und ihre Strahlenwege können sich im Gewebe überkreuzen. Es entsteht also eine vergleichsweise homogene Bestrahlung aller maligner Zellen unabhängig von ihrer Antigenexpression, welches als "Kreuzfeuereffekt" bezeichnet wird und die Entstehung von Rezidiven verhindern soll (Trümper et al. 2007). In einer systematischen Übersichtsarbeit über RIT bei NHL vergleicht Christos Emmanouilides die beiden zur Behandlung von NHL zugelassenen Radioimmunkonjugate ${ }^{90} \mathrm{Y}-\mathrm{IT}$ und ${ }^{131} \mathrm{I}-\mathrm{T}$ bezüglich der physikalischen und biologischen Eigenschaften ihrer Nuklide, welche in Tabelle 1.5 zusammengefasst dargestellt sind.

\begin{tabular}{|l|c|c|}
\hline & ${ }^{90}$ Y-IT & ${ }^{131}$ I-T \\
\hline Antikörper & Ibritumomab & Tositumomab \\
\hline Radionuklid & ${ }^{90}$ Yttrium & ${ }^{131}$ Iod \\
\hline Konjugation & Verbindung mittels Tiuxetan & Iodierung (kovalente Bindung) \\
\hline Strahlung & $\beta-S t r a h l u n g$ & $\beta-$ und $\gamma$-Strahlung \\
\hline Energie der $\beta-S t r a h l u n g$ & $2,3 \mathrm{MeV}$ & $0,6 \mathrm{MeV}$ \\
\hline Gewebedurchdringung & $5,3 \mathrm{~mm}$ & 0,8 mm \\
\hline Halbwertszeit & 64 Stunden & 8 Tage \\
\hline
\end{tabular}

Tabelle 1.5: Gegenüberstellung der Eigenschaften von ${ }^{90} \mathrm{Y}-\mathrm{IT}$ und ${ }^{131} \mathrm{I}-\mathrm{T}$; modifiziert nach Emmanouilides (2007, S.45). 
Daraus wird ersichtlich, dass der therapeutisch wirksame Anteil an radioaktiver Strahlung von

${ }^{131} \mathrm{I}$, nämlich die $\beta$-Strahlung, eine recht geringe Energie von $0,6 \mathrm{MeV}$ und demzufolge auch nur eine Gewebedurchdringung von $0,8 \mathrm{~mm}$ aufweist. Die Halbwertszeit des Isotops ist mit acht Tagen jedoch sehr lang, wobei die biologische Halbwertszeit des Konjugats ${ }^{131} \mathrm{I}-\mathrm{T}$ bei unterschiedlichen Patienten sehr variabel und schwer einschätzbar ist. Es besteht außerdem die Gefahr einer ungewollten Anreicherung von ${ }^{131} \mathrm{I}$ in der Schilddrüse. Aus diesen Gründen resultiert die Notwendigkeit einer genauen Dosimetrie und Radioprotektion bei der klinischen Anwendung. ${ }^{90}$ Y-IT dagegen trägt den hochenergetischen $(2,3 \mathrm{MeV}) \beta$-Strahler ${ }^{90} \mathrm{Y}$, welcher eine längere Gewebedurchdringung von 5,3 mm erreicht und zu dem weiter oben erwähnten "Kreuzfeuereffekt" führt. Durch den schnellen radioaktiven Zerfall bei einer Halbwertszeit von 64 Stunden besteht hier eine geringere Strahlenbelastung als bei ${ }^{131}$ I für das umliegende Gewebe (Emmanouilides 2007). Dies und die fehlende $\gamma$-Strahlung ermöglicht sogar eine ambulante Anwendung von ${ }^{90} \mathrm{Y}$-IT, während Patienten, die mit ${ }^{131} \mathrm{I}$-T behandelt werden, zur Verabreichung der RIT stationär aufgenommen und sogar isoliert werden müssen. Um ihre Umgebung vor einer Strahlenexposition zu schützen, müssen diese Patienten auch bestimmte Sicherheitsrichtlinien, wie zum Beispiel Isolation besonders von Schwangeren und Kindern oder sorgfältige Entsorgung von Körperflüssigkeiten, einhalten (Vose 2004).

\subsubsection{Klinische Anwendung der Radioimmuntherapie}

Als erstes Radioimmuntherapeutikum zur Therapie maligner Lymphome wurde ${ }^{90}$ Y-IT 2002 in den USA und 2004 in Europa zugelassen. Die Anwendung war ursprünglich für die Behandlung rezidivierter oder refraktärer indolenter oder follikulärer NHL sowie nach Rituximabtherapie refraktärer follikulärer Lymphome (FL) indiziert. Kürzlich wurde die Indikationsstellung um eine Konsolidierungstherapie mit RIT nach Erreichen einer Remission von vorher unbehandelten FL erweitert. ${ }^{131} \mathrm{I}-\mathrm{T}$ ist dagegen nur in den USA zur Therapie rezidivierter oder refraktärer indolenter, follikulärer oder transformierter NHL zugelassen (Gisselbrecht et al. 2009; Gregory et al. 2009). Der Einsatz der RIT bei aggressiven Lymphomen ist Gegenstand klinischer Studien, deren Ergebnisse im Verlauf näher beschrieben werden sollen.

Als Voraussetzung für die Applikation von ${ }^{90}$ Y-IT müssen die Patienten bestimmte klinische Kriterien erfüllen. So darf keine vorherige Knochenmark- oder Stammzelltransplantation stattgefunden haben und eine Infiltration des Knochenmarks durch das Lymphom sollte nicht mehr als 25,0\% betragen. Um einer möglichen schwerwiegenden Myelosuppression 
vorzubeugen, ist die Behandlung von Patienten mit weniger als 1.500/ $\mu$ l Neutrophilen Granulozyten oder weniger als 100.000/ $\mu 1$ Thrombozyten kontraindiziert. Auch bei bekannten hypersensiblen Reaktionen gegen murine Antikörper oder gegen andere Bestandteile der Therapie kann eine Behandlung selbstverständlich nicht durchgeführt werden.

Das Applikationsschema der RIT beinhaltet eine zweimalige Vorbehandlung mit einer relativ niedrigen Dosis Rituximab $\left(250 \mathrm{mg} / \mathrm{m}^{2}\right)$ eine Woche sowie einige Stunden vor der eigentlichen Verabreichung des Radioimmunokonjugats, welche ambulant durchgeführt werden kann. Dies dient dazu, freie, im Blut zirkulierende oder in der Milz liegende B-Zellen, welche das CD20-Antigen tragen, zu binden und vor der RIT zu schützen, sodass diese hauptsächlich an Tumorzellen wirken kann. Daraufhin wird ${ }^{90} \mathrm{Y}-\mathrm{IT}$ abhängig vom Körpergewicht bei normaler Thrombozytenanzahl mit einer Dosis von $0,4 \mathrm{mCi} / \mathrm{kg}$ Körpergewicht und bei leichter Thrombozytopenie (100.000-150.000/ $\mu$ l) mit nur noch einer 75-prozentigen Dosis von 0,3 mCi/kg Körpergewicht infundiert (Chapuy et al. 2007; Emmanouilides 2007).

Eine klinische Studie mit 349 Patienten zur Analyse des Sicherheitsprofils von ${ }^{90}$ Y-IT bei NHL zeigte, dass die primär auftretende und dosislimitierende unerwünschte Arzneimittelwirkung eine vorübergehende Myelosuppression war, die mit einem Abfall der Neutrophilen Granulozyten, der Thrombozyten und auch der Erythrozyten einherging. Bei einer Dosis von 0,4 mCi/kg Körpergewicht ${ }^{90} \mathrm{Y}$-IT erlitten 30,0 \% der Patienten eine Grad-IV-Neutropenie (Neutrophile Granulozyten $<500 / \mu 1$ ), $10,0 \%$ eine Grad-IV-Thrombozytopenie (Thrombozyten $<10.000 / \mu \mathrm{l})$ und $3,0 \%$ eine Grad-IV-Anämie $(\mathrm{Hb}<6,5 \mathrm{~g} / \mathrm{dl})$. Eine Anwendung von $0,3 \mathrm{mCi} / \mathrm{kg}$ Körpergewicht in einem kleineren Patientenkollektiv bewirkte prozentual jeweils häufiger eine Neutropenie, Thrombozytopenie und Anämie. Des Weiteren stellte sich heraus, dass eine Korrelation zwischen einer höheren Knochenmarkbeteiligung und einer größeren Wahrscheinlichkeit einer Grad-IV-Zytopenie besteht. Die Tiefpunkte der einzelnen Zellreihen traten sieben bis neun Wochen nach Anwendung der RIT auf und regenerierten sich innerhalb einer bis vier Wochen. Nicht hämatologische Nebenwirkungen, wie körperliche Schwäche, Übelkeit und Erbrechen, Kopfschmerzen oder Fieber waren mild (Grad I-II) und eher auf die Vortherapie mit Rituximab, bei dessen Anwendung diese Reaktionen typisch sind, zurückzuführen. Desgleichen gründeten sich leichte (Grad I-II) infusionsassoziierte Reaktionen wohl auch auf die vorherige Rituximabgabe. Insgesamt zeigte ${ }^{90}$ Y-IT bei Berücksichtigung der Kontraindikationen ein akzeptables Sicherheitsprofil für die Anwendung bei NHL (Witzig et al. 2003). 


\subsubsection{Radioimmuntherapie bei diffus großzelligen B-Zell-Lymphomen}

Mehrere Studien haben gezeigt, dass die Anwendung einer RIT bei rezidivierten oder refraktären indolenten oder follikulären NHL sowie nach Rituximabtherapie refraktärer FL signifikant höhere Ansprechraten als der Therapiestandard erreicht, welche auch nach Langzeituntersuchungen erhalten bleiben (Emmanouilides et al. 2007; Witzig et al. 2002). Ebenso konnten Morschhauser et al. zeigen, dass eine Konsolidierungstherapie mit RIT nach Erreichen einer Remission von vorher unbehandelten FL hoch effizient und ohne schwerwiegende Toxizität durchführbar ist (Morschhauser et al. 2008). Durch diese Erkenntnisse lässt sich die Zulassung von ${ }^{90} \mathrm{Y}$-IT in diesen Bereichen begründen.

Die Anwendung von RIT bei DLBCL ist dagegen derzeit noch nicht zugelassen und beschränkt sich somit auf klinische Studien und "Off-label Use".

Eine einleitende Studie, in der die Sicherheit und Wirksamkeit von RIT bei CD20-positiven B-Zell-NHL untersucht wurde, konnte auch bei DLBCL gute Therapie- und Langzeitergebnisse demonstrieren. Es zeigte sich für Patienten mit rezidivierten oder refraktären DLBCL nach durchschnittlich zweifacher Polychemotherapie eine gesamte Ansprechrate (overall response rate, ORR) nach Applikation von ${ }^{90} \mathrm{Y}$-IT von 58,0\% mit einer CR bei 33,0\% und einer unbestätigten kompletten Remission (CR unconfirmed, CRu) bei $17,0 \%$ der Patienten. Zwei von fünf Patienten wiesen sogar noch nach fünf Jahren eine beständige Remission auf (Gordon et al. 2004). Eine größere multizentrische Studie evaluierte die Anwendbarkeit von ${ }^{90} \mathrm{Y}$-IT bei älteren, für eine autoSZT ungeeigneten Patienten mit rezidivierten oder refraktären DLBCL. Auch hierbei wurden hohe Ansprechraten, die ebenfalls länger als zwei Jahre Bestand hatten, sowohl für Patienten mit primär chemotherapiesensiblem als auch chemotherapierefraktärem Lymphom erreicht (Morschhauser et al. 2007). Mit der Absicht, die Effektivität der RIT auch als Erstlinientherapie zu zeigen, führten Zinzani et al. eine Phase-II-Studie für unbehandelte ältere DLBCL-Patienten mit sechs Zyklen CHOP-21, gefolgt von einer einmaligen ${ }^{90}$ Y-IT-Applikation sechs bis zehn Wochen später, durch. Diese Kombination erreichte eine ORR von 100,0\% wobei die CR-Rate von 75,0 \% nach CHOP auf 95,0\% nach CHOP und RIT gesteigert wurde. Vier der fünf Patienten, die nach CHOP zu einer partiellen Remission (PR) gelangt waren, erzielten nach CHOP und RIT eine CR (Zinzani et al. 2008). Aufbauend auf diesen Ergebnissen und denen, die die Überlegenheit von R-CHOP gegenüber CHOP widerspiegeln, wurde kürzlich die Durchführbarkeit von sechs Zyklen R-CHOP-21 und nachfolgender RIT mit ${ }^{90} \mathrm{Y}$-IT bei älteren Patienten mit einem intermediär hohen oder hohen Risiko ohne vorheriger 
Behandlung untersucht. Auch hierbei konnte durch Verbesserung der CR-Raten und Abnahme an Progressen (progressive disease, PD) nach R-CHOP und RIT im Vergleich zu lediglich R-CHOP die Wirksamkeit, Durchführbarkeit und auch die Sicherheit dieses Therapieprotokolls gezeigt werden (Zinzani et al. 2010).

Wie weiter oben beschrieben, stellt eine autoSZT in Kombination mit einer HDC eine hoffnungsvolle, jedoch noch nicht etablierte Therapieoption für rezidivierte, chemotherapiesensible, aggressive NHL dar. Auf Grund der Intensität und Toxizität sowie des schlechten Nebenwirkungsprofils ist dieses Regime jedoch auf die Behandlungsmöglichkeit von jüngeren Patienten limitiert. RIT mit ${ }^{90}$ Y-IT ist im Gegensatz zu einer HDC jedoch auch für ältere Patienten gut verträglich und erzielt ähnlich gute ORR und dauerhafte Remissionen wie bei jüngeren Patienten.

Das geringe Ausmaß an unerwünschten Nebenwirkungen, welches bei der Behandlung älterer Patienten auftritt, ist ebenfalls mit dem jüngerer Patienten zu vergleichen (Emmanouilides et al. 2007). Es wurde daher auch die Durchführbarkeit von RIT in Zusammenhang mit Stammzelltransplantation untersucht:

Mehrere Studien zeigten, dass ${ }^{90}$ Y-IT zusammen mit dem HDC-Konzept BEAM, bestehend aus den Zytostatika Carmustin (BCNU®), Etoposid, Cytarabinosid (Alexan®) und Melphalan, als Konditionierung vor einer autoSZT eine effektive Alternative zur Behandlung rezidivierter oder refraktärer DLBCL darstellt, ohne jedoch die Toxizität oder das Risiko zur Entstehung von Sekundärtumoren der alleinigen HDC zu steigern (Krishnan et al. 2008; Shimabukuro-Vornhagen et al. 2008; Shimoni et al. 2007). Weiterhin konnte gezeigt werden, dass dieses Konzept der RIT in Kombination mit HDC vor autoSZT auch mit einer myeloablativen Hochdosis von ${ }^{90} \mathrm{Y}$-IT $(0,8-1,2 \mathrm{mCi} / \mathrm{kg}$ Körpergewicht) durchführbar und tolerabel ist, ohne eine zusätzliche Gefährdung für den Patienten zu generieren (Devizzi et al. 2008; Nademanee et al. 2005).

Zusätzlich zu den therapeutischen Ansätzen von RIT als Konditionierung vor autoSZT liegen auch erste Studien zur Anwendung von RIT nach einem Misserfolg von HDC mit autoSZT vor. Es wurde demonstriert, dass mehrfach vorbehandelte NHL-Patienten, die nach HDC und autoSZT einen Progress erlitten, sicher und effektiv mit ${ }^{90} \mathrm{Y}$-IT behandelt werden können (Jacobs et al. 2005; Vose et al. 2007). 


\subsection{Ziele der Arbeit}

Zusammenfassend kann festgehalten werden, dass die heute gültigen Therapiestandards zur Behandlung von DLBCL in den letzten Jahren zwar schon weitreichende und vielversprechende Entwicklungen durchgemacht haben, jedoch in manchen Bereichen weiterhin verbesserungswürdig sind. Besonders die Behandlung von Patienten mit höherem IPI oder solchen, die älter als 60 Jahre alt sind, chemotherapierefraktäre Lymphome oder Rezidive aufweisen, stellen die behandelnden Ärzte immer wieder vor Probleme. Die noch nicht zur Anwendung bei DLBCL zugelassene RIT könnte einige davon lösen, wie durch die weiter oben genannten Studien bereits angedeutet wurde. Dennoch sind noch weitere Untersuchungen zur Etablierung der klinischen Anwendung von RIT vonnöten, um mehr Informationen über Wirkung und Nebenwirkung zu sammeln und neue Therapierichtlinien zu schaffen.

Die vorliegende Arbeit beschäftigt sich mit der Erfassung und Analyse von Patientendaten, die anhand eines weltweiten internetbasierten Registers erhoben wurden. Es handelt sich dabei um DLBCL-Patienten, welche außerhalb klinischer, randomisierter Studien mit RIT behandelt wurden. Es soll gezeigt werden, dass die Anwendung von RIT auch im klinischen Alltag erfolgversprechend und sicher ist und zudem eine neue Therapieoption für DLBCLErkrankungen darstellt, bei denen die aktuellen Therapieleitlinien nicht zum Erfolg führten. Dabei spiegelt das Registerpatientenkollektiv durch seine Größe und Vielfältigkeit die tatsächliche klinische Anwendung von RIT bei DLBCL besser wider, als die zumeist kleinen und homogenen Studienkollektive, welche die Effizienz und auch unerwünschten Arzneimittelwirkungen der RIT somit möglicherweise nicht deutlich zeigen können.

Die in der vorliegenden Arbeit erzielten Ergebnisse und Auswertungen können zur Planung von zukünftigen Studien wichtige Hinweise liefern und unterstützend dazu beitragen, dass eine Zulassung der RIT bei DLBCL näher untersucht und in Betracht gezogen wird. Ein langfristiges Ziel stellt hier die Aufnahme der RIT in die aktuellen Therapieleitlinien zur Behandlung von DLBCL dar. 


\section{Material und Methoden}

\subsection{Internationales Radioimmuntherapie-Netzwerk}

Mit der Absicht, Daten von Langzeitüberwachungen von Patienten, welche weltweit außerhalb klinischer Studien mit einer RIT behandelt wurden, zu sammeln und auswerten zu können, wurde im Dezember 2006 das Internationale RIT-Netzwerk der Georg-AugustUniversität Göttingen als Zusammenschluss verschiedener nationaler Register gegründet. Es handelt sich dabei um ein von der Ethikkommission der Georg-August-Universität Göttingen geprüftes, internationales und internetbasiertes Register, welches Patientendaten aus momentan 14 verschiedenen Ländern verwaltet und untersucht. Das RIT-Netzwerk erhält finanzielle Unterstiutzung von "Bayer HealthCare". Die Beaufsichtigung der Datensätze, welche in einer zentralen Datenbank gespeichert werden, sowie die Verantwortung für die Aufrechterhaltung, Qualität und Sicherheit der Datenbank obliegen einem professionellen, unabhängigen Auftragsforschungsinstitut (Alcedis $\mathrm{GmbH}$ ) in Deutschland. Dieses Auftragsforschungsinstitut richtet sich dabei nach international anerkannten Standards und Richtlinien wie beispielsweise den Regulierungen der amerikanischen "Food and Drug Administration" zur Nutzung computergesteuerter Systeme in klinischen Studien.

Der internationale wissenschaftliche Aufsichtsrat des RIT-Netzwerkes, der durch die beiden internationalen Vorstandsvorsitzenden Prof. Dr. med. Trümper und Prof. Dr. med. Zinzani vertreten wird, ist verantwortlich für die Betreuung des Registers und entscheidet über die Durchführung von auf dem gesamten Datenkollektiv basierenden Analysen und Publikationen. Auf nationaler Ebene wird das Register von einem durch die teilnehmenden Wissenschaftler des Landes gewählten Vorsitzenden geführt, welcher die Entscheidung über eine Durchführbarkeit der Auswertung inländischer Datensätze trifft.

Die dieser Arbeit zugrunde liegenden Daten über radioimmuntherapierte DLBCL-Patienten entstammen diesem internationalen Register. 


\subsubsection{Datenerfassung}

Das RIT-Register erfasst Patientencharakteristika sowie Therapie- und Langzeitergebnisse von Patienten, welche außerhalb klinischer Studien mit RIT behandelt werden, sodass die tatsächliche weltweite klinische Anwendung von RIT bei malignen Lymphomen, die sich teilweise von vorgeschriebenen Leitlinien unterscheidet, besser einschätzbar und durchsichtiger wird. Um im Folgenden Aussagen über die Anwendbarkeit und Toxizität von RIT bei malignen Lymphomen über klinische Studien hinaus treffen zu können, werden in dem Register durch die partizipierenden Ärzte von jedem Patienten Daten über allgemeine, anamnestische Informationen, Vorerkrankungen und Vortherapien, Details über den Ablauf der RIT sowie Verlaufs- und Abschlussbeurteilungen, in denen unerwünschte Arzneimittelwirkungen erfasst werden, zusammengetragen. Vorgegebene elektronische Erhebungsbögen (siehe Kapitel 7.3, S.103) erleichtern den teilnehmenden Ärzten die Aufnahme ihrer Patientendaten in das Register und bewirken einen möglichst großen Konsens der registrierten Kernaussagen.

Die gesammelten Patientendaten werden anonymisiert in der zentralen Datenbank gespeichert und können nur mittels Benutzererkennung mit Passwortabfrage durch zugangsberechtigte Mitglieder des RIT-Netzwerks eingesehen werden. Die Zugangsberechtigung zur zentralen Datenbank sowie die entsprechenden Bearbeitungs- und Eingabemöglichkeiten richten sich nach dem Prinzip der geringsten Rechte, so dass jedes Mitglied lediglich auf individuell zugelassene Patientendaten Zugriff hat.

\subsubsection{Patienteneinwilligung und Sicherheit}

Vor einer Aufnahme der Patientendaten in das RIT-Register haben alle Patienten eine Aufklärung über den Ablauf, Nutzen und Datenschutz bei der Speicherung und Auswertung therapiebezogener Daten im Rahmen des Registers erhalten und eine schriftliche Einverständniserklärung unterzeichnet.

Die Ethikkommission der Georg-August-Universität Göttingen hat den Aufbau des RITRegisters geprüft und genehmigt, da sie keine ethischen oder rechtlichen Bedenken hatte. 


\subsection{Patienten mit diffus großzelligem B-Zell-Lymphom}

Die Aufnahme von Lymphompatienten in das Register des RIT-Netzwerks ist unabhängig von der Entität ihres malignen Lymphoms, sodass jegliche Varianten zu finden sind. Bei Abschluss der Datenerhebung für die vorliegende Arbeit waren 1.110 Patienten in das Register aufgenommen. Durch die bestehende klinische Zulassung von ${ }^{90} \mathrm{Y}-\mathrm{IT}$ zur Behandlung von FL stellen diese die am häufigsten erfasste Form der NHL dar, während DLBCL mit einem Patientenanteil von 19,5\% $(\mathrm{n}=215)$ aus neun verschiedenen Ländern vertreten sind (siehe Tabelle 2.1).

\begin{tabular}{|l|c|c|}
\hline Land & $\begin{array}{c}\text { Patienten, gesamt } \\
{[\mathbf{n}]}\end{array}$ & $\begin{array}{c}\text { Patienten, vollständig } \\
{[\mathbf{n}]}\end{array}$ \\
\hline Argentinien & 4 & 0 \\
\hline Deutschland & 23 & 20 \\
\hline Frankreich & 14 & 4 \\
\hline Israel & 2 & 35 \\
\hline Italien & 50 & 19 \\
\hline Österreich & 37 & 3 \\
\hline Polen & 25 & $\mathbf{1 3 7}$ \\
\hline Schweiz & $\mathbf{2 1 5}$ & 51 \\
\hline Südkorea & 56 & 3 \\
\hline Gesamt & & 3 \\
\hline
\end{tabular}

Tabelle 2.1: Anzahl und Herkunftsland der vollständig erhobenen und der gesamten Patientendaten, die für diese Analyse genutzt wurden. 
Auf den Daten dieser 215 an DLBCL erkrankten Patienten, die vom RIT-Netzwerk erfasst wurden, gründet sich die Analyse und Auswertung der vorliegenden Arbeit. 137 (63,7 \%) der Datensätze waren dabei zum Zeitpunkt der Verfassung dieser Arbeit bereits vollständig erhoben, wobei nur solche Erhebungsbögen mit mindestens drei dokumentierten Nachfolgeuntersuchungen und einem definierten Therapieende als vollständig gewertet wurden (siehe Tabelle 2.1). Damit der Datensatz als vollständig gewertet wurde, war des Weiteren eine elektronische Unterschrift vonnöten, mit der die teilnehmenden Ärzte die Richtigkeit und Vollständigkeit der angegebenen Daten bestätigen. Die restlichen, bislang unvollständig dokumentierten 78 Patienten (36,3\%), sollen weiterhin dokumentiert und zu Nachfolgeuntersuchungen einbestellt werden.

Es ist möglich, jedoch auf Grund der Anonymität der Patientendaten nicht bekannt, dass die Patienten neben der vorliegenden Registeranalyse auch in etwaigen klinischen Studien der teilnehmenden Ärzte des RIT-Netzwerkes untersucht wurden.

Der Zeitraum der Datenerhebung erstreckt sich über vier Jahre von der Gründung des RITNetzwerks im Dezember 2006 bis zum 14. Dezember 2010.

\subsection{Datenauswertung}

Die bis zum Ende des Erhebungszeitraums in die elektronischen Erhebungsbögen eingetragenen Daten wurden gesichert und nach unterschiedlichen Gesichtspunkten und Methoden ausgewertet.

Dabei wurden zuerst die Charakteristika und der Krankheitsstatus aller 215 Patienten (Gesamtkollektiv) analysiert und beschrieben. Danach erfolgte eine weitere Auswertung der Charakteristika und des Krankheitsstatus einer besondere Gruppe aus dem Gesamtkollektiv, der "First line"-Patienten, welche die RIT als Erstlinientherapie erhalten hatten. Es wurden hierbei die absoluten als auch die relativen Zahlen angegeben, wobei sich die relativen Zahlen, falls nicht anders beschrieben, immer auf das jeweils untersuchte gesamte Patientenkollektiv (Gesamtkollektiv oder "First line"-Patienten) beziehen. Weiterhin wurde für die verschiedenen Auswertungen stets der Median bestimmt, da sich dieser gegenüber ausreißenden Daten stabiler als der Mittelwert verhält.

Desgleichen wurde auch das ausgewertete Therapieansprechen, welches den Verlaufsbeurteilungen zu entnehmen war, für beide Patientenkollektive durch absolute sowie relative Zahlen beschrieben und das Verteilungsverhältnis anhand von Kreisdiagrammen dargestellt. 
Der Langzeitverlauf dagegen wurde mittels Kaplan-Meier-Analysen bezüglich des Gesamtüberlebens (overall survival, OS), der progressfreien Zeit (time to progression, TTP) sowie des progressfreien Überlebens (progression-free survival, PFS) untersucht. Bei den hierbei analysierten Patientenkollektiven handelt es sich nicht nur um das Gesamtkollektiv und die "First line"-Patienten, sondern zusätzlich auch um das "Relapse"-Patientenkollektiv, welches der Differenz von Gesamtkollektiv und "First line"-Kollektiv entspricht, sowie weiteren Subgruppen der "First line"-Patienten, welche sich in bestimmten Eigenschaften unterscheiden. In diesen Kurven nach Kaplan-Meier wurden sowohl zensierte Beobachtungen mit einem Kreuz bezeichnet und die Anzahl nichtzensierter lebender, beziehungsweise progressfreier Patienten ("patients at risk") als auch die mediane Nachuntersuchungszeit graphisch dargestellt. Der jeweilige Median des OS, TTP und PFS der verschiedenen Analysen ist definiert als Zeitdauer, bis die Überlebenswahrscheinlichkeit nach Kaplan-Meier $50,0 \%$ unterschreitet. Des Weiteren wurden die unterschiedlichen Überlebensraten nach einem, zwei und drei Jahren anhand der Kaplan-Meier-Analysen berechnet und als relative Zahl mit entsprechendem Konfidenzintervall, definiert als Bereich, welcher mit 95prozentiger Wahrscheinlichkeit den wahren Wert enthält, beschrieben. Die Auswertung des sowohl medianen als auch prozentualen Überlebens erfolgte, um im weiteren Verlauf eine bessere Vergleichbarkeit mit klinischen Studien zu bieten.

Ferner wurde das OS, die TTP und das PFS der verschiedenen Gruppen und Subgruppen anhand von log-Rank-Tests analysiert. Das Signifikanzniveau lag dabei gemäß dem internationalen Standard bei 0,05.

Die Erfassung und Verarbeitung aller Patientendaten wurde mithilfe des Programms "SAS Version 9.2" des Softwareunternehmens SAS Institute (Statistical Analysis Systems) realisiert. 


\subsection{Herausforderungen der Datenerhebung}

Die Daten, die für die vorliegende Arbeit erhoben wurden, stammen von Patienten, welche außerhalb klinischer, randomisierter Studien und außerhalb zugelassener Indikation mit RIT behandelt wurden. Dadurch konnte zwar eine große Anzahl mit RIT behandelter Patienten aus dem klinischen Alltag ausgewertet werden, es ergaben sich jedoch auch einige Erschwernisse und Ungenauigkeiten im Vergleich zu kontrollierten klinischen Studien.

Es ergibt sich, dass, auf Grund heterogener Einschlusskriterien, ungenau bestimmter Maßstäbe beim Therapieansprechen, fehlender Randomisierung und der Abhängigkeit vom jeweils behandelnden Arzt, die Validität der Registerdaten eingeschränkt gegenüber solchen aus kontrollierten klinischen Studien ist. Missverständliche, unglaubwürdige und auch mutmaßlich inkorrekte Angaben entstanden durch fehlendes Monitoring der Dateneingabe und die fehlende Kontrollmöglichkeit über die Richtigkeit der Daten. Die Nichtverfügbarkeit bestimmter Daten und der Mangel an Dokumentation von eingetretenen Ereignissen limitiert besonders die Aussagekraft der Überlebenskurven und Langzeitergebnisse. In der Diskussion der ausgewerteten Ergebnisse wird auf diese Problematik erneut eingegangen (siehe Kapitel 4.1.2, S.66).

In Folge dieser Schwierigkeiten der Datenerhebung mussten insgesamt 17 Patienten aus der Auswertung ausgeschlossen werden. Ausschlaggebend dafür war bei fünf dieser Patienten, neben anderen fehlenden Angaben, das Fehlen einer dokumentierten RIT. Ohne diese Informationen war eine Auswertung dieser Patienten nicht möglich. Die weiteren zwölf Patienten wurden auf Grund inkongruenter Patientendaten und der daraus resultierenden Unsicherheit über die Richtigkeit der im RIT-Register dokumentierten Daten aus der Auswertung genommen. Es lagen schriftliche Angaben zu vorangegangenen Therapien und dem Ansprechen der Patienten auf diese Vortherapien sowie auch auf die gegebene RIT vor, welche sich nicht mit den Angaben im RIT-Register decken.

Des Weiteren wurden 26 Patienten von einer Auswertung bezüglich der erreichten Granulozyten-Nadire und 15 Patienten bezüglich der erreichten Leukozyten-Nadire ausgeschlossen, da der jeweils dokumentierte Nadir von 0 Zellen/ $\mu$ l höchst unwahrscheinlich und mutmaßlich aus einer versehentlichen Fehleingabe in den elektronischen Erhebungsbögen resultiert. 


\section{Ergebnisse}

\subsection{Charakteristika der Patienten und ihrer Erkrankung}

Das in dieser Arbeit ausgewertete Patientenkollektiv, bestehend aus 215 teilnehmenden Patienten, zeigt eine gleichmäßige Geschlechterverteilung von 107 (49,8\%) männlichen und $108(50,2 \%)$ weiblichen Patienten. In einem Durchschnittsalter von 62 Jahren (minimal 17 bis maximal 88 Jahre) wurde bei ihnen erstmalig ein DLBCL diagnostiziert. Der Großteil dieser Patienten $(\mathrm{n}=120 ; 55,8 \%)$ war dabei bereits 60 Jahre und älter, während nur 15,8 \% ( $n=34)$ jünger als 40 Jahre und 28,4\% $(n=61)$ zwischen 40 und 60 Jahren alt waren (siehe Tabelle 3.1).

\begin{tabular}{|c|c|c|}
\hline & Anzahl [n] & Prozent [\%] \\
\hline Patienten & 215 & 50,2 \\
\hline+ & 108 & 49,8 \\
\hline ^ & 107 & \\
\hline $\begin{array}{c}\text { Alter [Jahre] } \\
\text { bei Erstdiagnose }\end{array}$ & & 15,8 \\
\hline$<20-<40$ & 34 & 28,4 \\
\hline $40-<60$ & 61 & 55,8 \\
\hline $60->80$ & 120 & \\
\hline
\end{tabular}

Tabelle 3.1: Charakterisierung des Patientenkollektivs.

Die Patienten wurden zum Diagnosezeitpunkt in die vier verschiedenen Stadien der AnnArbor-Klassifikation eingeteilt. Rund ein Drittel der Patienten $(n=68 ; 31,6 \%)$ hatte dabei DLBCL im Stadium IV und nur geringfügig weniger Patienten $(n=60 ; 27,9 \%)$ im Stadium III. Das restliche Drittel $(\mathrm{n}=70 ; 32,6 \%)$ wurde den Stadien I $(\mathrm{n}=16 ; 7,5 \%)$ und II $(\mathrm{n}=54$; 
$25,1 \%)$ zugeteilt. Sechs Patienten $(2,8 \%)$ hatten einen Befall extranodaler Strukturen und 11 Patienten $(5,1 \%)$ wurde kein Tumorstadium zugeordnet.

Unter Berücksichtigung der fünf Risikofaktoren 'Alter $\geq 60$ Jahre', 'Tumorausbreitung nach Ann-Arbor im Stadium III oder IV', 'Befall von mehr als einer extranodalen Struktur', 'schlechter Allgemeinzustand' und 'erhöhter Serumspiegel der LDH' wurde die Prognose für rund zwei Drittel des Patientenkollektivs $(\mathrm{n}=144 ; 67,0 \%)$ mit Hilfe des IPI eingeschätzt. Bei dem restlichen Drittel der Patienten $(\mathrm{n}=71 ; 33,0 \%)$ war der IPI auf Grund fehlender Dokumentation eines oder mehrerer Risikofaktoren nicht zu berechnen. Es hatten somit 35 Patienten (16,3\%) des Gesamtkollektivs vor Beginn der RIT ein niedriges Risiko, denn sie erfüllten höchstens eines der IPI-Kriterien. 52 (24,2\%) beziehungsweise 30 (14,0\%) Patienten wurde ein niedrigintermediäres oder intermediär hohes Risiko zugeteilt. Bei ihnen waren zwei respektive drei der Risikofaktoren aufgetreten. Ein hohes Risiko war für 27 der Patienten $(12,5 \%)$ zu erwarten, da $25(11,6 \%)$ von ihnen vier der Risikofaktoren und zwei Patienten $(0,9 \%)$ sogar alle fünf Risikofaktoren aufwiesen.

Bei 186 Patienten (87,0 \%) wurde im Register eine Knochenmarkinfiltration verneint, für 22 Patienten $(10,2 \%)$ lagen diesbezüglich keine Daten vor und sechs Patienten $(2,8 \%)$ hatten einen dokumentierten Befall des Knochenmarks. Dieser war bei drei Patienten größer als 25,0 \%, was laut Zulassung eine Dosisreduktion der RIT erfordert.

In den meisten Fällen $(\mathrm{n}=162 ; 75,3 \%)$ wurden die Lymphome nicht weiter in ihre histologischen Subtypen eingeteilt, sondern lediglich als DLBCL aufgeführt. Bei 37 Patienten wurde jedoch eine weitere histologische Einteilung durchgeführt und diese ergab für 27 Patienten $(12,6 \%)$ ein zentroblastisches Lymphom, für neun Patienten $(4,0 \%)$ ein großzelliges anaplastisches Lymphom und bei einem Patienten $(0,5 \%)$ ein immunoblastisches Lymphom. Zusätzlich wurden ein Lymphom (0,5\%) als DLBCL vom intravaskulären Typ und 15 Lymphome (7,0\%) als DLBCL vom mediastinalen Typ klassifiziert. Diese Auswertung des Krankheitsstatus des Patientenkollektivs vor der RIT ist in Tabelle 3.2 zusammengefasst. 


\begin{tabular}{|c|c|c|}
\hline Tumorstadium & Anzahl [n] & Prozent $[\%]$ \\
\hline $\mathrm{E}$ & 6 & 2,8 \\
\hline I & 16 & 7,5 \\
\hline II & 54 & 25,1 \\
\hline III & 60 & 27,9 \\
\hline IV & 68 & 31,6 \\
\hline n.d. & 11 & 5,1 \\
\hline \multicolumn{3}{|l|}{ Internationaler Prognostischer Index } \\
\hline Niedriges Risiko & 35 & 16,3 \\
\hline Niedrigintermediäres Risiko & 52 & 24,2 \\
\hline Intermediär hohes Risiko & 30 & 14,0 \\
\hline Hohes Risiko & 27 & 12,5 \\
\hline n.d. & 71 & 33,0 \\
\hline \multicolumn{3}{|l|}{ Knochenmarkinfiltration } \\
\hline Nein & 187 & 87,0 \\
\hline $\mathrm{Ja}$ & 6 & 2,8 \\
\hline Befall $<25 \%$ & 3 & 1,4 \\
\hline Befall > 25\% & 3 & 1,4 \\
\hline n.d. & 22 & 10,2 \\
\hline \multicolumn{3}{|l|}{ Histologische Subtypen } \\
\hline DLBCL & 162 & 75,3 \\
\hline - Zentroblastisch & 27 & 12,6 \\
\hline - Großzellig anaplastisch & 9 & 4,1 \\
\hline - Immunoblastisch & 1 & 0,5 \\
\hline DLBCL, mediastinaler Typ & 15 & 7,0 \\
\hline DLBCL, intravaskulärer Typ & 1 & 0,5 \\
\hline
\end{tabular}

n.d. $=$ nicht dokumentiert

Tabelle 3.2: Krankheitsstatus des Patientenkollektivs vor der Radioimmuntherapie. 
Vor beziehungsweise im Rahmen der jeweiligen radioimmuntherapeutischen Behandlung hatte das Patientenkollektiv ein unterschiedliches Spektrum an Vortherapien erhalten, welches Tabelle $3.3 \mathrm{zu}$ entnehmen ist. Somit waren insgesamt 46 Patienten (21,4\%) strahlentherapeutisch anbehandelt. Die meisten $(n=34 ; 15,8 \%)$ davon hatten einmalig eine Strahlentherapie erhalten, während zwölf Patienten $(5,6 \%)$ zwei oder drei Mal bestrahlt worden sind.

Bei fast allen Patienten, insgesamt 209 (97,2 \%), sind eine oder mehrere Chemotherapien als Vortherapie dokumentiert. Zwischen einer und drei Therapielinien wurden bei 187 Patienten $(87,0 \%)$ angewendet, während 21 Patienten $(9,7 \%)$ vor der RIT bereits vier bis sechs Chemotherapien erhalten hatten. Lediglich bei einem Patienten $(0,5 \%)$ waren mehr als sieben vorangegangene Chemotherapien beschrieben. Der monoklonale anti-CD20-Antikörper Rituximab war dabei bei 185 Patienten $(86,0 \%)$ als Monotherapie oder in Kombination mit Chemotherapeutika ein Bestandteil der vorangegangenen Therapielinien.

24 Patienten $(11,1 \%)$ wurden bereits vor der RIT stammzelltransplantiert und 15 Patienten $(7,0 \%)$ wurden schon zuvor einmalig mit einer RIT behandelt. 


\begin{tabular}{|c|c|c|}
\hline \multicolumn{3}{|c|}{ Strahlentherapie } \\
\hline Therapielinien & Anzahl [n] & Prozent [\%] \\
\hline 1 & 34 & 15,8 \\
\hline 2 & 7 & 3,3 \\
\hline 3 & 5 & 2,3 \\
\hline n.d. & 169 & 78,6 \\
\hline \multicolumn{3}{|c|}{ Chemotherapie } \\
\hline Therapielinien & Anzahl [n] & Prozent [\%] \\
\hline $1-3$ & 187 & 87,0 \\
\hline $4-6$ & 21 & 9,7 \\
\hline$\geq 7$ & 1 & 0,5 \\
\hline n.d. & 6 & 2,8 \\
\hline \multicolumn{3}{|c|}{ Stammzelltransplantation } \\
\hline Therapielinien & Anzahl [n] & Prozent $[\%]$ \\
\hline 1 & 24 & 11,1 \\
\hline n.d. & 191 & 88,9 \\
\hline \multicolumn{3}{|c|}{ Vorherige RIT } \\
\hline Therapielinien & Anzahl [n] & Prozent [\%] \\
\hline 1 & 15 & 7,0 \\
\hline n.d. & 200 & 93,0 \\
\hline
\end{tabular}

n.d. $=$ nicht dokumentiert

Tabelle 3.3: Spektrum an vorherigen Therapielinien des Gesamtkollektivs. 


\subsection{Indikationsstellung für die Radioimmuntherapie und ihre Anwendung}

Für insgesamt 171 Patienten (79,5\% des Gesamtkollektivs) der Analyse liegen dokumentierte Angaben bezüglich der Indikationsstellung für die durchgeführte RIT vor. Demnach hat beinahe die Hälfte dieser 171 Patienten $(n=79 ; 46,2 \%)$ die RIT als Erstlinientherapie erhalten, in den meisten Fällen $(n=62)$ als Konsolidierungstherapie nach vorangegangener Chemotherapie. Weitere neun Patienten erhielten als Erstlinientherapie die RIT zur Konditionierung im Rahmen einer Hochdosistherapie mit BEAM, für einen Patienten wurde eine primäre RIT als Indikation angegeben und für sieben Patienten andere, nicht weiter spezifizierte Indikationsstellungen dokumentiert.

33 Patienten (19,3\%) erhielten die RIT als Zweitlinientherapie, welche bei 20 Patienten als Konsolidierung gegeben wurde. Außerdem wurde sieben Patienten die RIT als Zweitlinientherapie nach einem Rezidiv, drei Patienten auf Grund von therapierefraktärer Erkrankung und drei Patienten aus anderer Indikation heraus verabreicht.

Auch bei 26 Patienten (15,2 \%) mit der RIT als Drittlinientherapie war die Konsolidierungstherapie nach vorangegangener Chemotherapie für insgesamt zehn Patienten die häufigste Indikation. Des Weiteren wurden acht Patienten in dieser Gruppe nach einem Rezidiv, sechs Patienten mit therapierefraktärem Lymphom und zwei Patienten zur Konditionierung vor BEAM mit RIT behandelt.

Als Viertlinientherapie oder mehr wurden 33 Patienten (19,3\%) mit RIT behandelt. Hierbei waren die Behandlung nach Rezidiv $(\mathrm{n}=13)$ und die Behandlung therapierefraktärer DLBCL ( $n=12)$ die häufigsten Indikationen. Nur bei vier Patienten war eine Konsolidierungstherapie, bei einem Patient eine Konditionierung vor Hochdosistherapie (Z-BEAM) und bei drei Patienten eine andere Indikation gegeben.

Die Verteilung der Therapielinien, in der die RIT der Registeranalyse verabreicht wurde, ist in Tabelle 3.4 dargestellt und die Indikationsstellung zur RIT in Bezug auf die verschiedenen Therapielinien ist in Abbildung 3.1 gezeigt.

Für die Anwendung der RIT wurde bei einem Großteil des gesamten Patientenkollektivs (n = 200; 93,0\%) ${ }^{90} \mathrm{Y}$-IT als genutztes Radioimmuntherapeutikum dokumentiert, wobei für 165 Patienten (76,7 \%) zusätzlich die eingesetzte Energiedosis aufgeführt wurde. Diese lag im Durchschnitt bei $0,34 \mathrm{mCi} / \mathrm{kg}$ Körpergewicht mit einer Verteilung zwischen 0,01 bis $0,67 \mathrm{mCi} / \mathrm{kg}$ Körpergewicht. 


\begin{tabular}{|c|c|c|}
\hline & $\begin{array}{c}\text { Anzahl dokumentierter } \\
\text { Patienten [n] }\end{array}$ & Prozent [\%] \\
\hline Erstlinientherapie & 79 & 46,2 \\
\hline Zweitlinientherapie & 33 & 19,3 \\
\hline Drittlinientherapie & 26 & 15,2 \\
\hline Viertlininentherapie und mehr & 33 & 19,3 \\
\hline Total & 171 & 100,0 \\
\hline
\end{tabular}

Tabelle 3.4: Verteilung der Therapielinien, in denen die RIT bei 171 dokumentierten Patienten des Gesamtkollektivs verabreicht wurde.

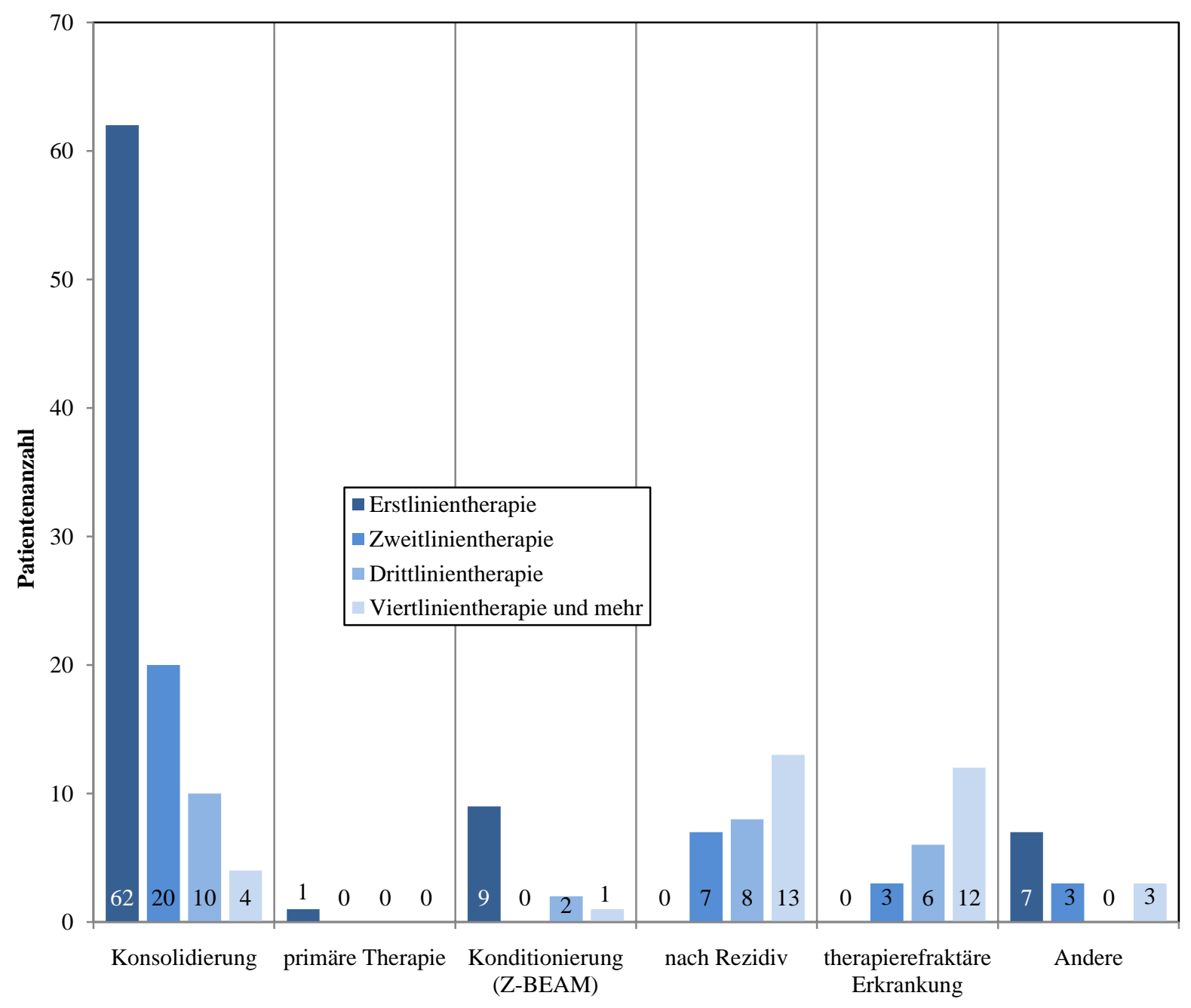

Abbildung 3.1: Indikation zur Radioimmuntherapie nach Therapielinie. 
Zur besseren Übersicht werden im Folgenden nur die Patienten mit der Indikation zur Konsolidierung, primärer Therapie oder Konditionierung in Erstlinientherapie als "First line" ( $\mathrm{n}=72 ; 33,5 \%$ des Gesamtkollektivs) bezeichnet, während alle anderen unter "Relapse" ( $\mathrm{n}=143 ; 66,5 \%$ des Gesamtkollektivs) zusammengefasst werden. Die Charakteristika der "First line"-Patienten werden im folgenden Kapitel ausführlich ausgewertet.

\subsection{1 "First line"-Patienten}

Als besonderes Patientenkollektiv wurde die Gruppe der "First line"-Patienten gesondert analysiert. Es handelt sich hierbei um 72 Patienten (33,5\% des Gesamtkollektivs), welche zuvor unbehandelt die RIT als Erstlinientherapie mit der Indikation zur Konsolidierung infolge einer Polychemotherapie, als primäre Therapie oder zur Konditionierung im Rahmen einer Hochdosistherapie mit BEAM erhalten hatten.

Wie auch beim gesamten Patientenkollektiv zeigte sich eine nahezu gleichmäßige Geschlechterverteilung mit 35 (48,6\%) männlichen und 37 (51,4\%) weiblichen Patienten. Zum Zeitpunkt der Erstdiagnosestellung der DLBCL waren diese Patienten durchschnittlich 65 Jahre alt, wobei der jüngste Patient 17 Jahre und der älteste 88 Jahre alt war. Bei der Analyse der Altersverteilung zeigte sich, dass ein Großteil $(n=50 ; 69,4 \%)$ der Patienten 60 Jahre und älter gewesen ist. Sieben $(9,7 \%)$ von ihnen waren sogar 80 Jahre und älter. Zehn Patienten $(13,9 \%)$ wurden der Altersgruppen zwischen 20 und 40 Jahre und zwölf Patienten $(16,7 \%)$ der Altersgruppe 40 bis 60 Jahre zugeteilt.

Die Einteilung des Tumorstadiums nach der eingangs beschriebenen Ann-ArborKlassifikation ergab für lediglich drei Patienten (4,2\%) DLBCL im Stadium I, für 20 Patienten $(27,8 \%)$ im Stadium II und für 18 Patienten (25,0\%) im Stadium III. Insgesamt 23 Patienten $(31,9 \%)$ waren an DLBCL im Tumorstadium IV erkrankt. Ein extranodaler Befall wurde hier für keinen Patienten beschrieben. Acht Patienten $(11,1 \%)$ wurde kein Tumorstadium zugeordnet.

Die Prognoseeinschätzung mittels des IPI wurde bei 61 Patienten $(84,2 \%)$ durchgeführt und dokumentiert. 14 Patienten $(19,4 \%)$ zeigten dabei ein niedriges Risiko (IPI =0-1) und 20 Patienten $(27,8 \%)$ ein niedrigintermediäres Risiko (IPI = 2). 13 Patienten $(18,0 \%)$ erfüllten drei und 14 Patienten $(19,5 \%)$ sogar vier bis fünf der IPI-Kriterien, so dass man bei ihnen ein intermediär hohes beziehungsweise ein hohes Risiko zu erwartet hatte. 
Eine Knochenmarkinfiltration wurde lediglich bei einem Patienten $(1,4 \%)$ beschrieben, während 67 Patienten (93,0 \%) keine Infiltration aufwiesen und bei vier Patienten $(5,6 \%)$ diesbezüglich keine Angaben gemacht wurden. Bei diesem einen Patienten waren 2,0 \% des Knochenmarks befallen und damit lag die Infiltration weit unter der Grenze $(25,0 \%)$ zur Dosisreduktion der folgenden RIT.

Des Weiteren wurde auch für die Gruppe der "First line"-Patienten eine histologische Einteilung in die unterschiedlichen Subtypen der DLBCL durchgeführt. Es zeigte sich, wie auch schon beim Gesamtkollektiv, ein Überwiegen der klassischen DLBCL, an denen 65 $(90,3 \%)$ Patienten erkrankt waren. Eine weitere histologische Unterteilung innerhalb dieser Gruppe erfolgte jedoch nicht. Die Diagnose DLBCL vom mediastinalen Typ wurde für sechs Patienten $(8,3 \%)$ gestellt, während bei einem Patienten $(1,4 \%)$ ein DLBCL vom intravaskulärem Typ vorlag. Tabelle 3.5 gibt eine Übersicht über die klinischen Charakteristika der analysierten "First line"-Patienten.

\begin{tabular}{|c|c|c|}
\hline & Anzahl [n] & Prozent [\%] \\
\hline Patienten & 72 & 100,0 \\
\hline+ & 37 & 51,4 \\
\hline o & 35 & 48,6 \\
\hline $\begin{array}{c}\text { Alter (Jahre) } \\
\text { bei Erstdiagnose }\end{array}$ & 10 & 13,9 \\
\hline$<20-<40$ & 12 & 16,7 \\
\hline $40-<60$ & 50 & 69,4 \\
\hline $60->80$ & & \\
\hline
\end{tabular}

Fortsetzung auf der folgenden Seite 


\begin{tabular}{|c|c|c|}
\hline Tumorstadium & Anzahl [n] & Prozent $[\%]$ \\
\hline I & 3 & 4,2 \\
\hline II & 20 & 27,8 \\
\hline III & 18 & 25,0 \\
\hline IV & 23 & 31,9 \\
\hline n.d. & 8 & 11,1 \\
\hline Internationaler Prognostischer Index & Anzahl [n] & Prozent $[\%]$ \\
\hline Niedriges Risiko & 14 & 19,4 \\
\hline Niedrigintermediäres Risiko & 20 & 27,8 \\
\hline Intermediär hohes Risiko & 13 & 18,1 \\
\hline Hohes Risiko & 14 & 19,4 \\
\hline n.d. & 11 & 15,3 \\
\hline \multicolumn{3}{|l|}{ Knochenmarkinfiltration } \\
\hline Nein & 67 & 93,0 \\
\hline $\mathrm{Ja}$ & 1 & 1,4 \\
\hline n.d. & 4 & 5,6 \\
\hline \multicolumn{3}{|l|}{ Histologische Subtypen } \\
\hline DLBCL & 65 & 90,3 \\
\hline DLBCL, mediastinaler Typ & 6 & 8,3 \\
\hline DLBCL, intravaskulärer Typ & 1 & 1,4 \\
\hline
\end{tabular}

n.d. $=$ nicht dokumentiert

Tabelle 3.5: Patienten- und Lymphomcharakteristika der "First line"-Patientengruppe. 
Wie die Definition der Gruppe der "First line"-Patienten bereits besagt, erhielten alle Patienten die RIT als Erstlinientherapie und waren somit zuvor unbehandelt. Dabei war einzig für einen Patienten $(1,4 \%)$ die RIT als primäre Therapie indiziert, während die meisten Patienten $(\mathrm{n}=62 ; 86,1 \%)$ zur Konsolidierung nach vorangegangener Chemotherapie mit RIT behandelt wurden. Das häufigste dabei angewandte Chemotherapieprotokoll war, entsprechend den aktuellen Therapieleitlinien (siehe Kapitel 1.1.3.1, S.11) bei 45 Patienten R-CHOP-21. Bei weiteren zwei Patienten wurden die vorherigen Protokolle (CHOP-21 beziehungsweise R-COP) im Verlauf ebenfalls auf R-CHOP-21 umgestellt und bei einem Patienten wurde das R-CHOP-21-Schema mit Ifosfamid kombiniert. Des Weiteren erhielten fünf Patienten CHOP-21 ohne Rituximab und drei Patienten R-CHOP-14. Die restlichen sechs Patienten erhielten andere Therapieprotokolle, welche Tabelle $3.6 \mathrm{zu}$ entnehmen sind. Insgesamt hatten also insgesamt 56 der Patienten, die RIT als Konsolidierung in Erstlinientherapie erhalten hatten $(n=62)$, Rituximab als Bestandteil ihrer Chemotherapie erhalten und lediglich sechs Patienten nicht.

\begin{tabular}{|c|c|c|c|c|}
\hline \multicolumn{4}{|c|}{ Chemotherapieprotokoll } & Anzahl [n] \\
\hline \multicolumn{4}{|c|}{ R-CHOP-21 } & 45 \\
\hline \multicolumn{4}{|c|}{ CHOP-21 erweitert zu R-CHOP-21 } & 1 \\
\hline \multicolumn{4}{|c|}{ R-COP erweitert zu R-CHOP-21 } & 1 \\
\hline \multicolumn{4}{|c|}{ R-CHOP-21 kombiniert mit Ifosfamid } & 1 \\
\hline \multicolumn{4}{|c|}{ R-CHOP-14 } & 3 \\
\hline \multicolumn{4}{|c|}{ CHOP-21 } & 5 \\
\hline \multicolumn{5}{|c|}{ Andere: } \\
\hline \multicolumn{3}{|c|}{ R-CEOP } & & 1 \\
\hline \multicolumn{3}{|c|}{ CNOP } & & 1 \\
\hline \multicolumn{3}{|c|}{$\mathrm{R}-\mathrm{COP}$} & & 3 \\
\hline \multicolumn{3}{|c|}{ R-FOP } & & 1 \\
\hline $\mathrm{R}:$ & Rituximab & $\mathrm{C}:$ & Cyclophosp & \\
\hline H: & Doxorubicin (Hydroxyldaunorubicin) & O: & Vincristin $(\mathbf{C}$ & $n \circledast)$ \\
\hline P: & Prednison & E: & Etoposid & \\
\hline $\mathrm{N}:$ & Mitoxantron (Novatron $®)$ & F: & Fludarabin & \\
\hline
\end{tabular}

Tabelle 3.6: Angewandte Chemotherapieprotokolle der "First line"-Patienten bei Konsolidierung. 
Neun $(12,5 \%)$ der "First line"-Patienten erhielten die RIT zur Konditionierung vor einer Hochdosistherapie (Z-BEAM). Die dabei zur Vorbereitung angewandten Protokolle der konventionellen Vortherapien sind in Tabelle 3.7 dargestellt. Auch hier zeigt sich, dass fast alle Patienten $(\mathrm{n}=8)$ Rituximab als Bestandteil ihres Therapieprotokolls hatten.

\begin{tabular}{|l|c|}
\hline Chemotherapieprotokoll & Anzahl [n] \\
\hline R-CHOP-14 & 3 \\
R-CHOP-14 umgestellt auf R-DHAP & 2 \\
\hline CHOP-14 & 1 \\
\hline R-CHOP-21 umgestellt auf R-ESHAP & 1 \\
\hline R-ACBVP & 1 \\
R-ACBVP kombiniert mit hochdosiertem MTX & 1 \\
\hline
\end{tabular}

R: $\quad$ Rituximab

CHOP: $\quad$ Cyclophosphamid, Doxorubicin (Hydroxyldaunorubicin), Vincristin (Oncovin $\left.{ }^{\circledR}\right)$, Prednison

DHAP: Dexamethason, Cytarabin (Ara C), Cisplatin

ESHAP: $\quad$ Etoposid, Methylprednisolon, Cytarabin (Ara C), Cisplatin

ACBVP: $\quad$ Doxorubicin (Adriblastin®), Cyclophosphamid, Bleomycin, Vindesin,

MTX: $\quad$ Prednison

Tabelle 3.7: Chemotherapieprotokolle, welche bei den "First line"-Patienten mit einer RIT als Konditionierung vor BEAM als Vortherapien angewandt wurden.

${ }^{90} \mathrm{Y}$ wurde in $69(95,8 \%)$ der Fälle als genutztes Isotop dokumentiert. Die totale Strahlendosis, die dabei angewendet wurde, lag bei 51 (70,8\%) beschriebenen Anwendungen in einem Bereich zwischen minimal 0,01 und maximal 0,47 mCi/ $\mathrm{kg}$ Körpergewicht und im Median bei 0,35 mCi/kg Körpergewicht. 


\subsection{Klinisches Ergebnis nach Radioimmuntherapie}

Nach Durchführung der RIT wurden die Patienten zur Dokumentation des klinischen Ergebnisses $\mathrm{zu}$ verschiedenen Zeitpunkten $\mathrm{zu}$ Nachsorgeuntersuchungen eingeladen. Idealerweise sollte dies nach drei, sechs, zwölf, 24 und 36 Monaten geschehen, wobei im internationalen RIT-Register das jeweilige Formular der Verlaufsbeurteilung auszufüllen war.

Im Median wurden die Registerpatienten rund ein Jahr (11,5 Monate) dokumentiert nachuntersucht. Die späteste Verlaufsbeurteilung wurde nach über drei Jahren (38,2 Monate) durchgeführt, die frühsten direkt nach Verabreichung der RIT (0,0 Monate). Die mediane Nachuntersuchungszeit des Patientenkollektivs "First line" lag minimal höher bei 12,1 Monaten. Die späteste Nachuntersuchung wurde hier fast drei Jahre (35,4 Monate) und die frühste etwas mehr als einen Monat (1,3 Monate) nach der RIT durchgeführt. Insgesamt zeigt sich, dass im Verlauf immer weniger Nachuntersuchungen dokumentiert wurden und somit immer weniger "patients at risk" Informationen über das Langzeitverhalten der Patienten liefern. So wurde die 3-Monats-Verlaufsbeurteilung noch für 85,1\% (n=183) des Gesamtkollektivs beschrieben, während nur 73,0\% $(\mathrm{n}=157)$ und 51,6\% $(\mathrm{n}=111)$ der Verlaufsbeurteilungen nach sechs beziehungsweise zwölf Monaten vorlagen. Lediglich für 20,0 \% der Patienten $(n=43)$ wurde eine Nachuntersuchung nach 24 Monaten und für sogar nur $4,1 \%$ der Patienten $(n=9)$ eine Nachuntersuchung nach 36 Monaten dokumentiert. Die Auswirkungen davon auf die Interpretation der Langzeitergebnisse soll im späteren Verlauf der vorliegenden Arbeit diskutiert werden (siehe Kapitel 4.1.2, S.66).

\subsubsection{Therapieansprechen}

Bestandteil der jeweiligen Verlaufsbeurteilungen ist unter anderem die Dokumentation der für jeden Patienten besten Antwort nach erfolgter RIT. Hierbei zeigte sich, dass etwas mehr als die Hälfte der behandelten Patienten in der aktuellsten Nachuntersuchung ( $\mathrm{n}=117 ; 54,4 \%)$ eine CR des DLBCL und 19 weitere Patienten (8,9\%) eine PR erlangt hat. Insgesamt 136 Patienten des Gesamtkollektivs zeigten also ein Ansprechen auf die heterogenen Therapielinien in Kombination mit der RIT, was einer Gesamtansprechrate (overall response rate, ORR) von 63,3\% entspricht. Zwei Patienten (0,9\%) erreichten eine Stabilisierung ohne Fortschreiten der Erkrankung (stable disease, SD), während sich bei knapp einem Viertel der Verläufe ( $\mathrm{n}=51 ; 23,7 \%)$ ein Progress (progressive disease, PD) der DLBCL zeigte. Bei 26 Patienten $(12,1 \%)$ wurden diesbezüglich keine Angaben dokumentiert. 
Das Therapieansprechen nach Verabreichung der RIT wurde zusätzlich auch für die Patientenkollektive "First line" und "Relapse" gesondert ausgewertet, wobei zudem die "First line"-Patienten in Anlehnung des IPI in zwei Altergruppen (<60 Jahre und $\geq 60$ Jahre) unterteilt wurden.

Hier zeigt sich, dass die "First line"-Patienten $(\mathrm{n}=72)$ eine ORR von 83,3\% $(\mathrm{n}=60)$ aufweisen. Es wurden insgesamt $55 \mathrm{CR}(76,4 \%)$ und fünf PR (6,9\%) erreicht. Weiterhin erlitten neun Patienten (12,5\%) in dieser Gruppe einen Progress (PD) der DLBCL. Es lag kein dokumentierter Krankheitsstillstand (SD) vor, so dass für drei Patienten $(4,2 \%)$ entsprechend kein Therapieansprechen dokumentiert wurde.

Die 22 "First line"-Patienten (100,0\%), welche jünger als 60 Jahre waren, erreichten eine ORR von 81,8\% $(\mathrm{n}=18)$ mit anteilig $17 \mathrm{CR}(77,3 \%)$ und einer PR $(4,5 \%)$. Es wurden hier zwei PD $(9,1 \%)$ beobachtet. Bei zwei weiteren Patienten $(9,1 \%)$ wurde kein Therapieansprechen dokumentiert.

Auch bei den mindestens 60-jährigen Patienten $(n=50,100,0 \%)$ zeigte sich bei $38(76,0 \%)$ dokumentierten CR und vier $(8,0 \%)$ dokumentierten PR eine ORR von 84,0\% ( $\mathrm{n}=42)$. Des Weiteren wiesen sieben ältere Patienten $(14,0 \%)$ einen PD des Lymphoms auf. Bei einem Patienten $(2,0 \%)$ wurde entsprechend kein Therapieansprechen dokumentiert.

Diese Verteilungsmuster des Therapieansprechens aller "First line"-Patienten sowie in Abhängigkeit des Alters sind in Abbildung 3.2 und Abbildung 3.3 schematisch dargestellt. 


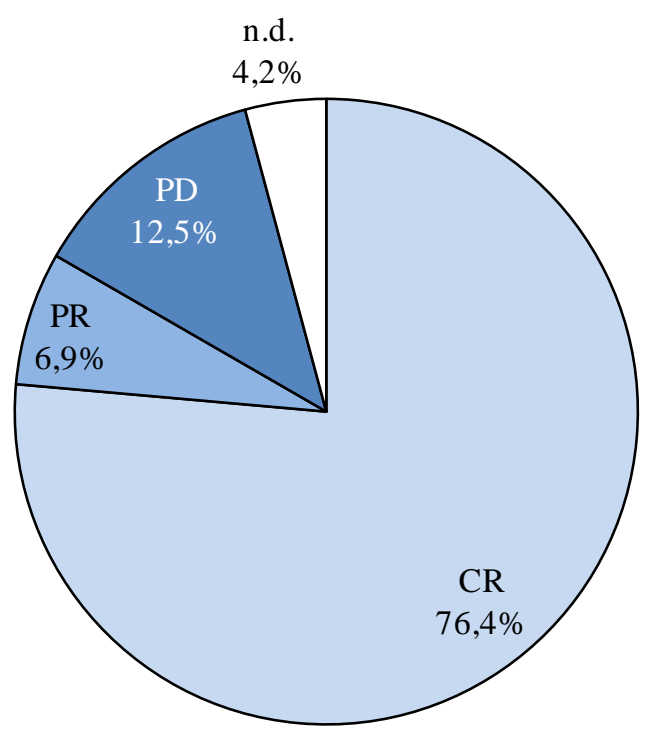

Abbildung 3.2: Häufigkeit der jeweils besten Therapieantwort nach Verabreichung der RIT beim "First line"-Patientenkollektiv.

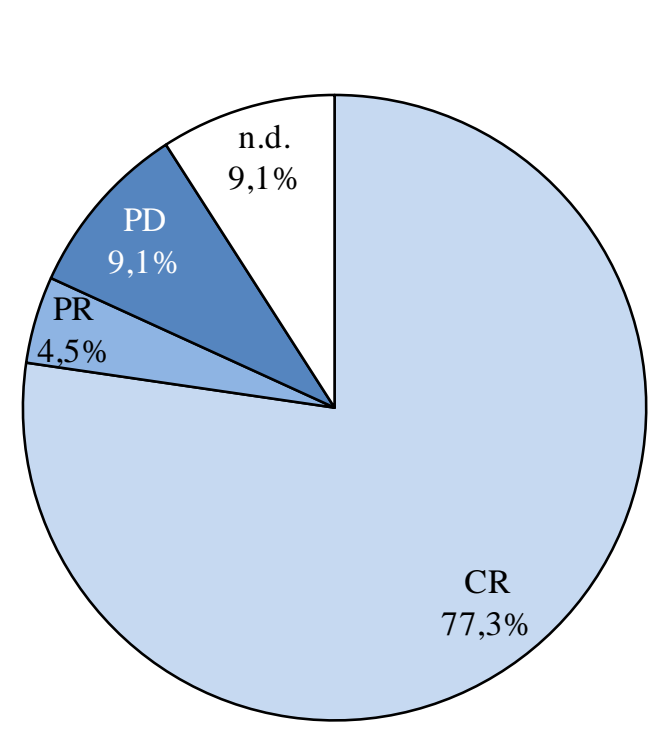

$<60$ Jahre

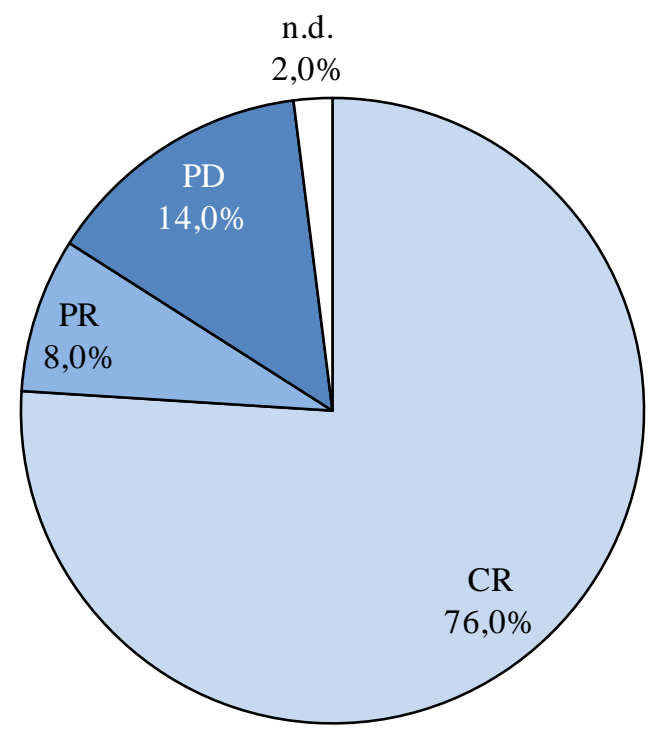

$\geq 60$ Jahre

Abbildung 3.3: Häufigkeit der jeweils besten Therapieantwort nach Verabreichung der RIT bei jüngeren "First line"-Patienten (links) und älteren "First line"-Patienten (rechts). 
Die 143 Patienten des "Relapse"-Kollektivs dagegen, welche mehrfach und heterogen vorbehandelt wurden, zeigen nach der zusätzlich verabreichten RIT eine ORR von lediglich $53,1 \%(\mathrm{n}=76)$. Es lagen dabei $62 \mathrm{CR}(43,3 \%)$ und 14 PR $(9,8 \%)$ vor. Des Weiteren wurden in dieser Gruppe zwei SD (1,4\%) und 42 PD (29,4\%) dokumentiert. Für 23 Patienten $(16,1 \%)$ wurde dagegen in dieser Gruppe kein Therapieansprechen dokumentiert. Abbildung 3.4 zeigt das Therapieansprechen des "Relapse"-Patientenkollektivs anhand eines Kreisdiagrammes.

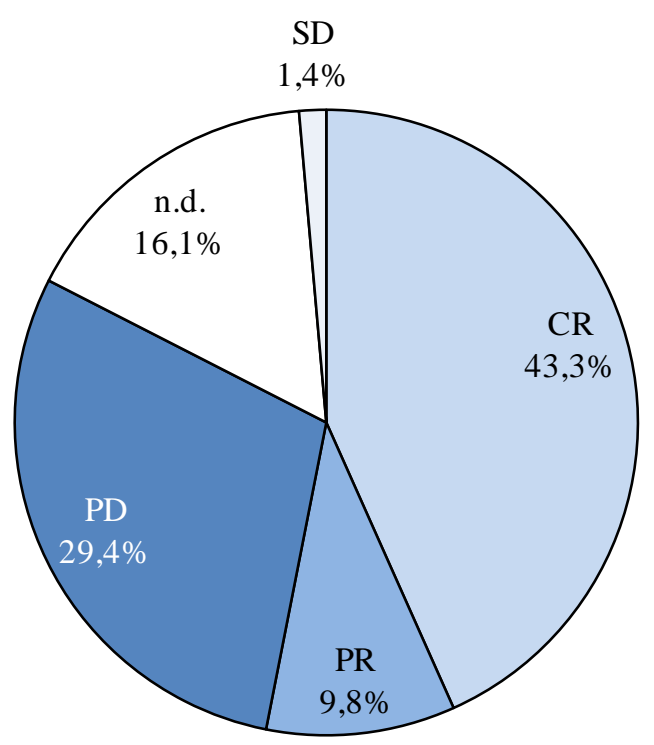

Abbildung 3.4: Häufigkeit der jeweils besten Therapieantwort nach Verabreichung der RIT beim "Relapse"-Patientenkollektiv.

\subsubsection{Gesamtüberleben}

Das Gesamtüberleben (OS) wurde für anfangs 201 Patienten (93,5\%) des Gesamtkollektivs bestimmt und mittels Kaplan-Meier-Analyse untersucht (siehe Abbildung 3.5). Im Verlauf der Nachuntersuchungszeit waren insgesamt 66 Patienten verstorben, so dass sich eine mediane Überlebenszeit von 27,2 Monaten ergibt. 53 dieser Patienten waren bereits im ersten Jahr nach RIT verstorben, zehn weitere im Verlauf des zweiten Jahres und die übrigen drei Patienten bis zur Vollendung des dritten Nachbeobachtungsjahres. Die Anzahl nichtzensierter überlebender Patienten (= "patients at risk") sank von ursprünglich 201 (93,5\%) auf 82 $(38,1 \%)$ nach zwölf Monaten, weiter auf 26 (12,1\%) nach 24 Monaten und letztendlich auf fünf Patienten (2,3\%) nach 36 Monaten. Somit konnte für die Patienten des RIT-Registers, deren Gesamtüberleben dokumentiert wurde, ein 1-Jahres-OS von 69,0\% 
$($ Konfidenzintervall $=\mathrm{KI}=61,7-75,7 \%)$, ein 2-Jahres-OS von 56,0 \% $(\mathrm{KI}=45,5-64,5 \%)$ sowie ein 3-Jahres-OS von 43,0 \% (KI = 27,9-57,3) errechnet werden (siehe Tabelle 3.8).

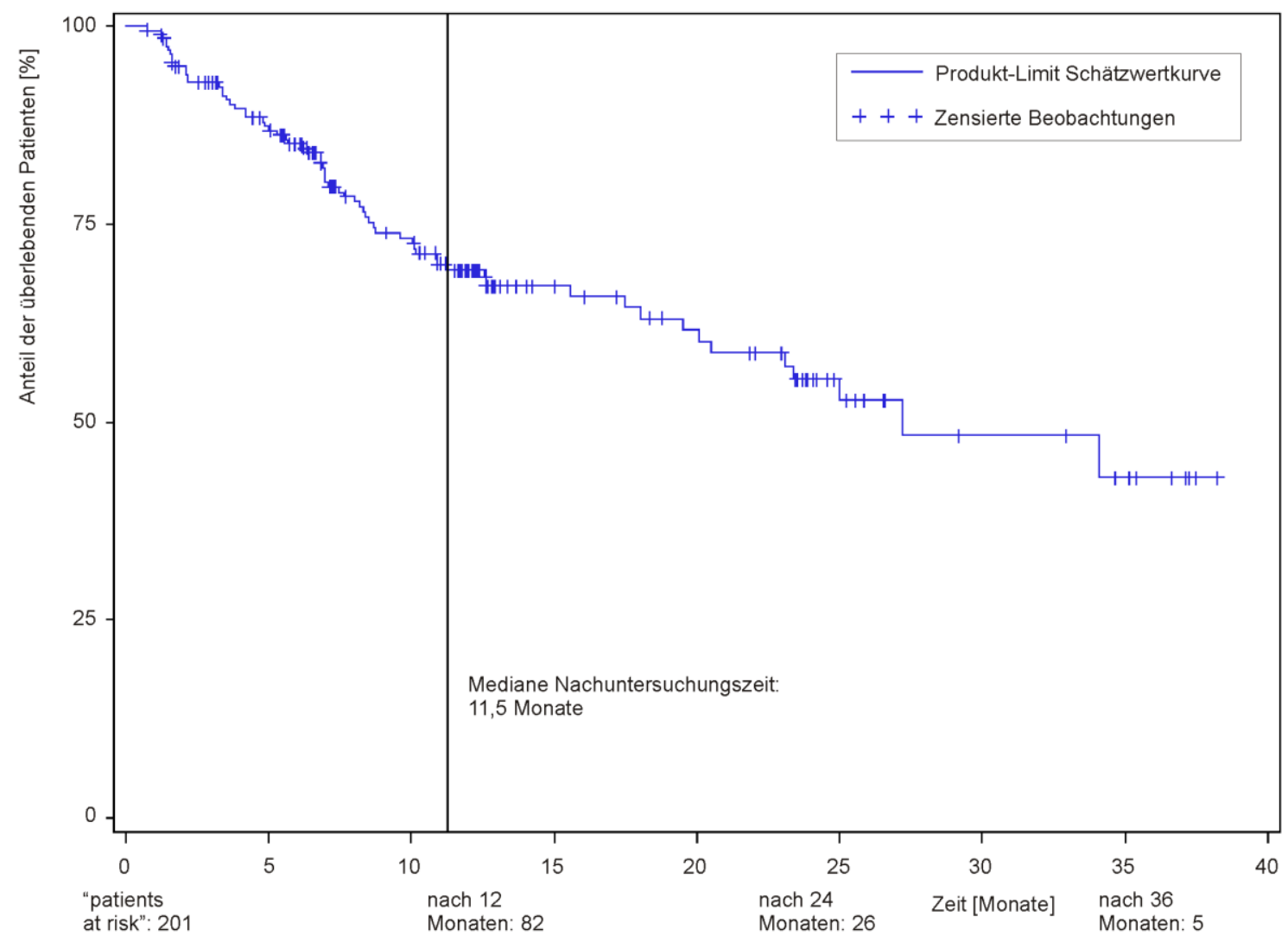

Abbildung 3.5: Gesamtüberleben (OS) des Gesamtkollektivs.

Eine vergleichende Kaplan-Meier-Untersuchung des OS der beiden Patientenkollektive "First line" und "Relapse" zeigte bei ursprünglich 72 analysierten "First line"-Patienten $(100,0 \%)$ mit 15 Todesfällen ein medianes Überleben von 34,1 Monaten. Im "Relapse"Patientenkollektiv wurden diesbezüglich zu Beginn 94 Patientendaten (65,7 \% von insgesamt 143 "Relapse"-Patienten) dokumentiert, wobei 37 Todesfälle eintraten. Daraus ergab sich eine mediane Überlebenszeit von 20,5 Monaten.

Der Vergleich der beiden Überlebenskurven ergibt im log-Rank-Test einen p-Wert $=0,0071$ und damit einen signifikanten Unterschied der beiden Patientenkollektive zu Gunsten der "First line"-Patienten. Diese zeigten besonders in den ersten zwei Nachuntersuchungsjahren ein besseres Gesamtüberleben als die analysierten "Relapse"-Patienten. Lediglich gegen Ende des dritten Jahres überkreuzen sich die Überlebenskurven, wobei hier jedoch keine "patients at risk" mehr beim "First line"-Kollektiv vorlagen (siehe Abbildung 3.6). 


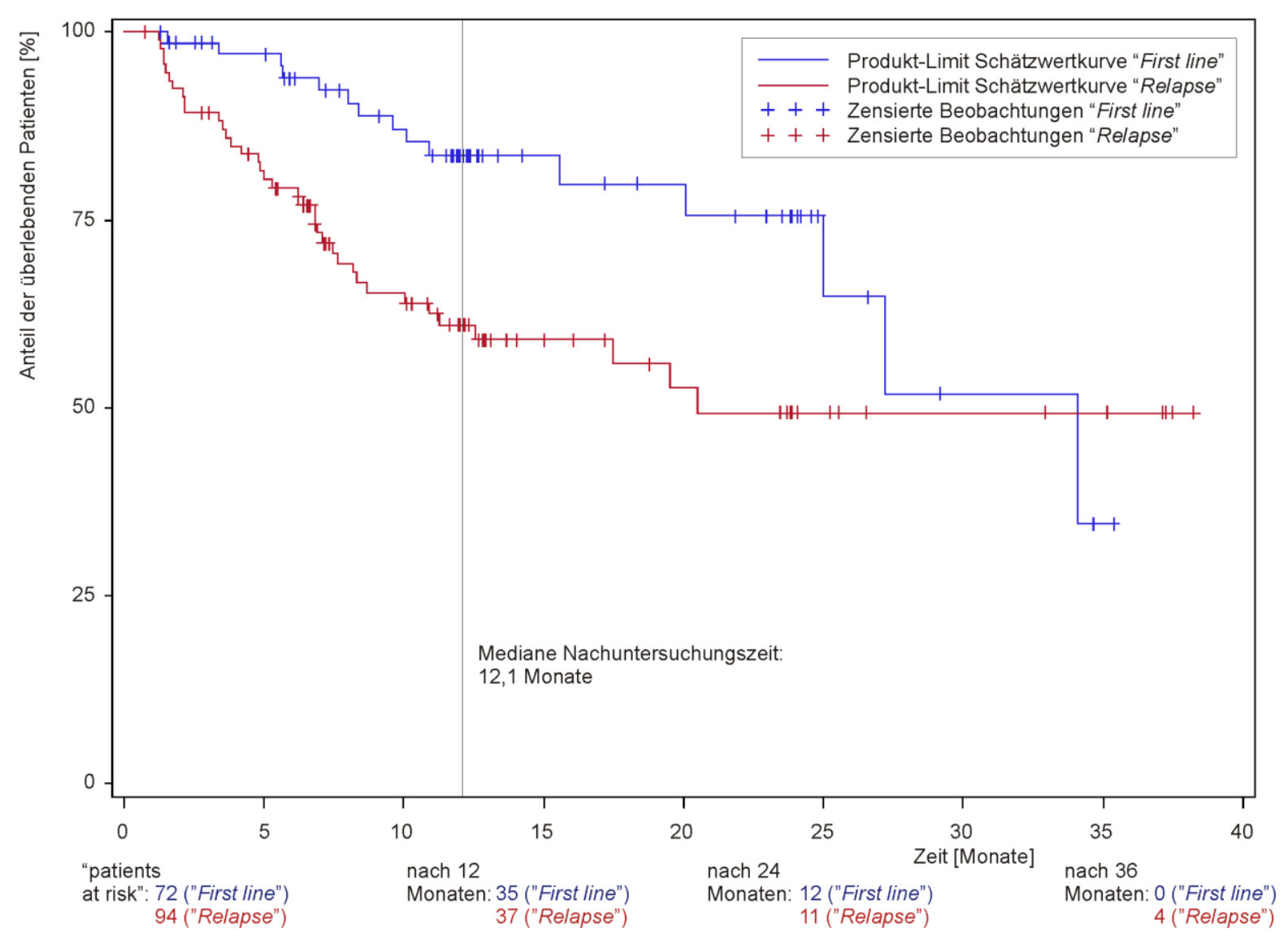

Abbildung 3.6: Gesamtüberleben (OS) der "First line"- und "Relapse"-Patienten im Vergleich.

Die höhere Wahrscheinlichkeit für ein Langzeitüberleben des "First line"-Patientenkollektivs gegenüber den "Relapse"-Patienten in den ersten zwei Nachuntersuchungsjahren spiegelt sich darüber hinaus auch in den OS-Raten wider. So erreichten die "First line"-Patienten ein 1-Jahres-OS von 84,0 \% $(\mathrm{KI}=71,6-90,0 \%)$ und ein 2-Jahres-OS von 76,0\% $(\mathrm{KI}=58,9$ $86,3 \%$ ), während die "Relapse"-Patienten ein 1-Jahres-OS von lediglich 61,0 \% (KI = 49,5$70,6 \%)$ und ein 2-Jahres-OS von 49,0\% $(\mathrm{KI}=35,3-62,0 \%)$ aufwiesen. Dagegen lag das 3-Jahres-OS der "First line"-Patienten bei 35,0\% (KI=7,0-65,5\%) im Vergleich zu wiederum 49,0 \% (KI = 35,3-62,0 \%) 3-Jahres-OS der "Relapse"-Gruppe (siehe Tabelle 3.8). 


\begin{tabular}{|l|c|c|c|}
\hline & $\begin{array}{c}\text { 1-Jahres-OS } \\
(\mathbf{K I}) \\
{[\%]}\end{array}$ & $\begin{array}{c}\text { 2-Jahres-OS } \\
(\mathbf{K I}) \\
{[\%]}\end{array}$ & $\begin{array}{c}\text { 3-Jahres-OS } \\
\text { (KI) } \\
{[\%]}\end{array}$ \\
\hline Gesamtkollektiv & $\begin{array}{c}69,0 \\
(61,7-75,7)\end{array}$ & $\begin{array}{c}56,0 \\
(45,5-64,5)\end{array}$ & $\begin{array}{c}43,0 \\
(27,9-57,3)\end{array}$ \\
\hline "First line"-Patienten & 84,0 & 76,0 & 35,0 \\
& $(71,6-90,0)$ & $(58,9-86,3)$ & $(7,0-65,5)$ \\
\hline "Relapse"-Patienten & 61,0 & 49,0 & 49,0 \\
& $(49,5-70,6)$ & $(35,3-62,0)$ & $(35,3-62,0)$ \\
\hline
\end{tabular}

Tabelle 3.8: Gesamtüberleben (OS) nach einem, zwei und drei Jahren nach RIT für das Gesamtkollektiv, sowie die Patientengruppen "First line" und "Relapse".

Für die Patienten der "First line"-Gruppe wurde das Gesamtüberleben überdies in kleineren Subgruppen, welche in Anlehnung an die drei Patientengruppen der aktuellen Therapieleitlinien (siehe Kapitel 1.1.3.1, S.11) in Abhängigkeit von Patientenalter, IPI und Rituximab als Bestandteil der Vortherapie aufgestellt worden sind, gesondert untersucht und tabellarisch für das erste, zweite und dritte Nachuntersuchungsjahr aufgeführt (siehe Tabelle 3.9).

Im Vergleich der Überlebenswahrscheinlichkeit von "First line"-Patienten in Abhängigkeit vom Alter (jünger als 60 gegenüber mindestens 60 Jahre) zeigte sich kein signifikanter Unterschied ( $\mathrm{p}=0,58$ im log-Rank-Test). Es waren hierbei anfangs 22 Patienten $(30,6 \%)$, welche jünger als 60 Jahre waren, dokumentiert, von denen sechs Patienten bei einem medianen Überleben von 34,1 Monaten verstorben waren. 50 untersuchte "First line"Patienten $(69,4 \%)$, welche 60 Jahre oder älter waren, wiesen bei neun Todesfällen eine mediane Überlebenszeit von 27,2 Monaten auf. Es ist auffällig, dass sich die Überlebenskurven nach Kaplan-Meier im Verlauf mehrfach kreuzen, wobei die jüngeren Patienten (blaue Kurve in Abbildung 3.7) im ersten Nachuntersuchungsjahr eine höhere Überlebenswahrscheinlichkeit aufweisen, die älteren Patienten dagegen im zweiten Jahr.

Des Weiteren ergab auch die Kaplan-Meier-Analyse der Überlebenswahrscheinlichkeit bezogen auf einen IPI von null bis eins verglichen mit der Wahrscheinlichkeit bei einem IPI von mindestens zwei keinen signifikanten Unterschied ( $p=0,85$ im log-Rank-Test). Es wurden zu Beginn 14 Patienten (19,4\%) mit einem IPI kleiner als zwei, bei denen die Überlebenswahrscheinlich nach fast drei Jahren Nachuntersuchungszeit noch über $50,0 \%$ lag 
und somit der Median noch nicht erreicht wurde, sowie 47 Patienten (65,3\%) mit erhöhtem Risiko (IPI $\geq 2$ ), deren medianes Überleben bei 27,2 Monaten lag, untersucht. In der Gruppe mit niedrigerem Risikoprofil waren lediglich drei Patienten im ersten Jahr nach RIT verstorben, danach trat kein weiterer Todesfall auf. Die Patienten der Subgruppe mit höherem Risiko verstarben dagegen auch im weiteren zeitlichen Verlauf der Dokumentation (insgesamt elf Patienten sind innerhalb von drei Nachuntersuchungsjahren verstorben). Es zeigt sich, dass die Kurven im Bereich der ersten zwei Nachuntersuchungsjahre ähnlich verlaufen und sich auch mehrfach überkreuzen (siehe Abbildung 3.8). Interessanterweise hatten die Patienten mit niedrigem Risiko innerhalb der ersten 20 Monate eine niedrigere (jedoch nicht signifikante) Überlebenswahrscheinlichkeit. Im weiteren Verlauf weisen sie jedoch eine höhere Überlebenswahrscheinlichkeit auf.

Zuletzt wurde das OS von ursprünglich 64 "First line"-Patienten (88,9\%) mit Rituximab als Bestandteil ihres Therapieprotokolls sowie von den sieben Patienten $(9,7 \%)$, die zuvor kein Rituximab erhalten hatten, mittels Kaplan-Meier-Analyse untersucht. Von den Rituximabpatienten waren bei einer medianen Überlebenszeit von 34,1 Monaten 14 Patienten im Verlauf verstorben, während bei den Patienten ohne Rituximab-Vortherapie kein Todesfall auftrat und somit auch kein medianes Überleben errechnet werden konnte. Die Kurvenverläufe zeigen einen Überlebensvorteil der Patienten ohne Rituximab gegenüber deren mit Rituximab, welcher jedoch nicht signifikant ist $(\mathrm{p}=0,14)$ (siehe Abbildung 3.9).

Es ist auffällig, dass die Anzahl der nichtzensierten überlebenden Patienten im Verlauf der Nachuntersuchungen stark abnimmt und in den beschriebenen Subgruppenanalysen der "First line"-Patienten nach 36 Monaten jeweils keine "patients at risk" mehr zur Verfügung standen. Des Weiteren weisen auch insbesondere die Konfidenzintervalle der verschiedenen 3-Jahres-OS eine breite Spanne auf (siehe Tabelle 3.9). 


\begin{tabular}{|c|c|c|c|c|}
\hline "First line"-Patienten & $\begin{array}{c}\text { 1-Jahres-OS (KI) } \\
{[\%]}\end{array}$ & $\begin{array}{c}\text { 2-Jahres-OS (KI) } \\
{[\%]}\end{array}$ & 3-Jah & $\begin{array}{l}\text { res-OS (KI) } \\
{[\%]}\end{array}$ \\
\hline < 60 Jahre & $82,0 \quad(53,0-93,7)$ & $58,0 \quad(24,1-81,4)$ & 29,0 & $(1,7-69,0)$ \\
\hline$\geq 60$ Jahre & $84,0 \quad(69,6-92,2)$ & $84,0 \quad(69,6-92,2)$ & 45,0 & $(8,0-77,6)$ \\
\hline $\mathrm{IPI}<2$ & $79,0 \quad(47,2-92,5)$ & $79,0 \quad(47,2-92,5)$ & 79,0 & $(47,2-92,5)$ \\
\hline IPI $\geq 2$ & $85,0 \quad(69,2-92,9)$ & $75,0 \quad(54,0-87,2)$ & 23,0 & $(1,4-60,7)$ \\
\hline mit Rituximab & $81,0 \quad(68,1-89,6)$ & $70,0 \quad(50,0-83,8)$ & 38,0 & $(7,7-68,8)$ \\
\hline ohne Rituximab & $100,0 \quad(100,0)$ & $100,0 \quad(100,0)$ & 100 & $(100,0)$ \\
\hline
\end{tabular}

Tabelle 3.9: Gesamtüberleben (OS) nach einem, zwei und drei Jahren nach RIT für das "First line"-Patientenkollektiv in Abhängigkeit von Patientenalter, IPI und Rituximab als Therapiebestandteil.

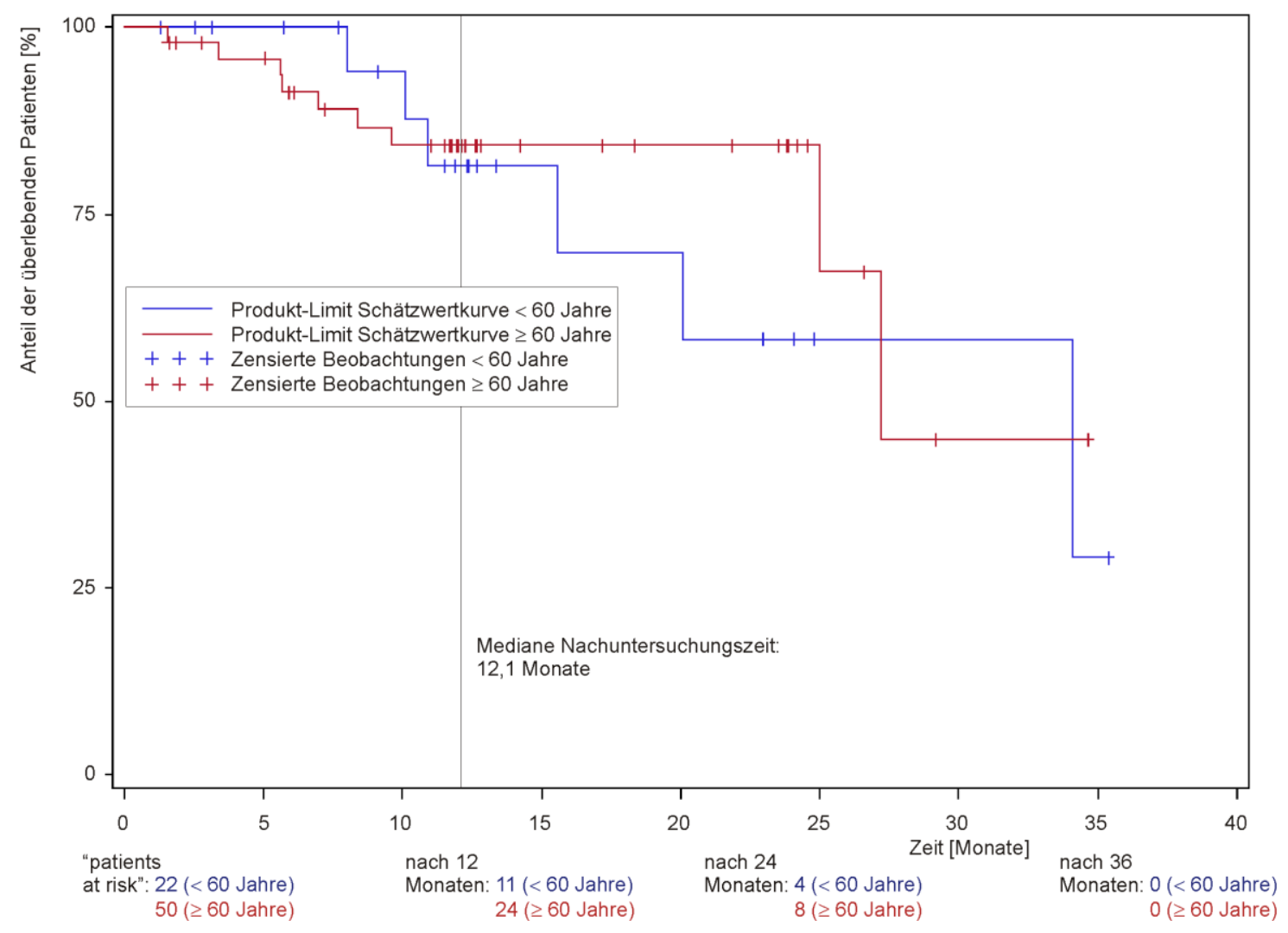

Abbildung 3.7: Gesamtüberleben (OS) der "First line"-Patienten in Abhängigkeit des Patientenalters. 


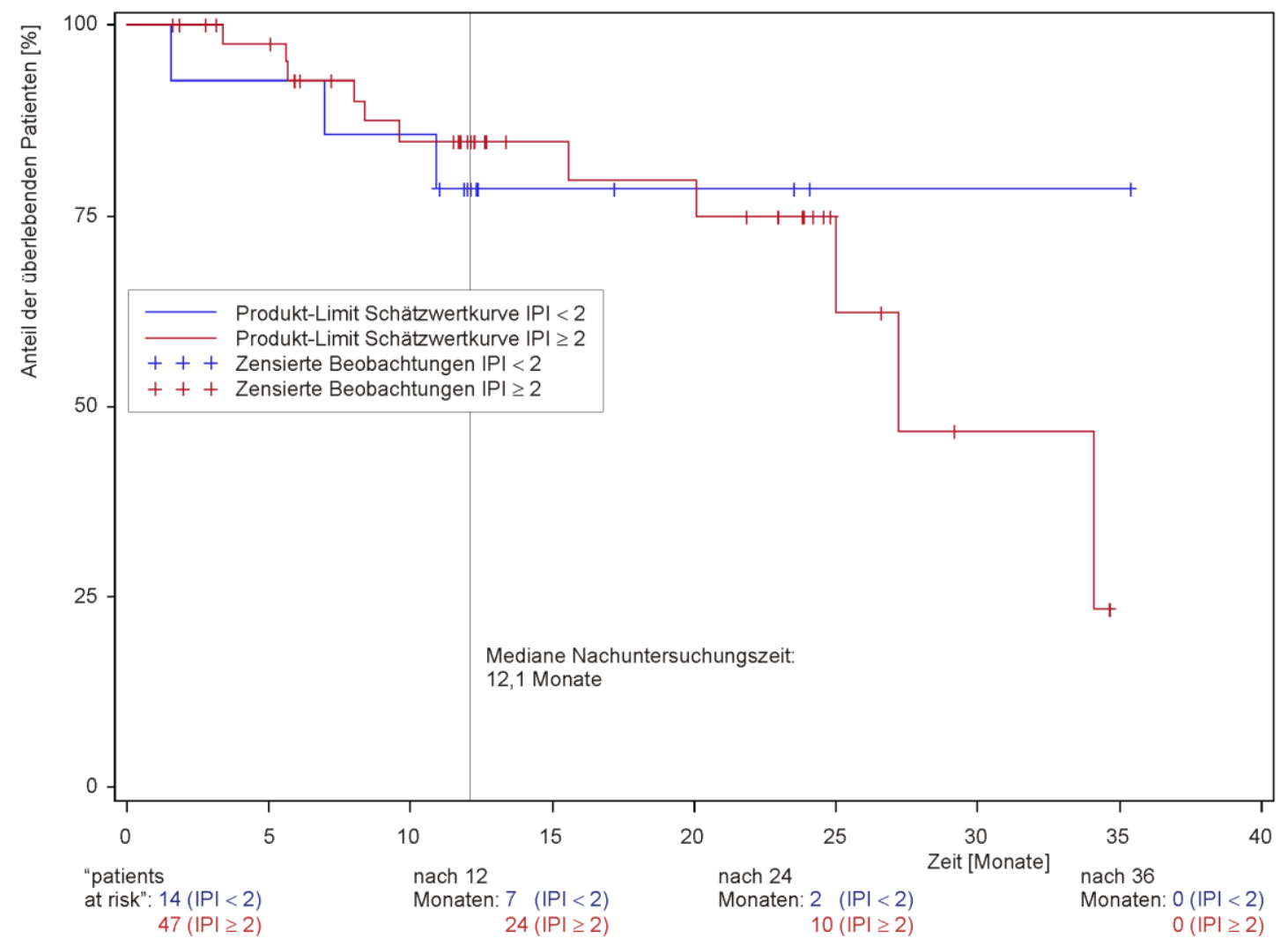

Abbildung 3.8: Gesamtüberleben (OS) der "First line"-Patienten in Abhängigkeit des IPI.

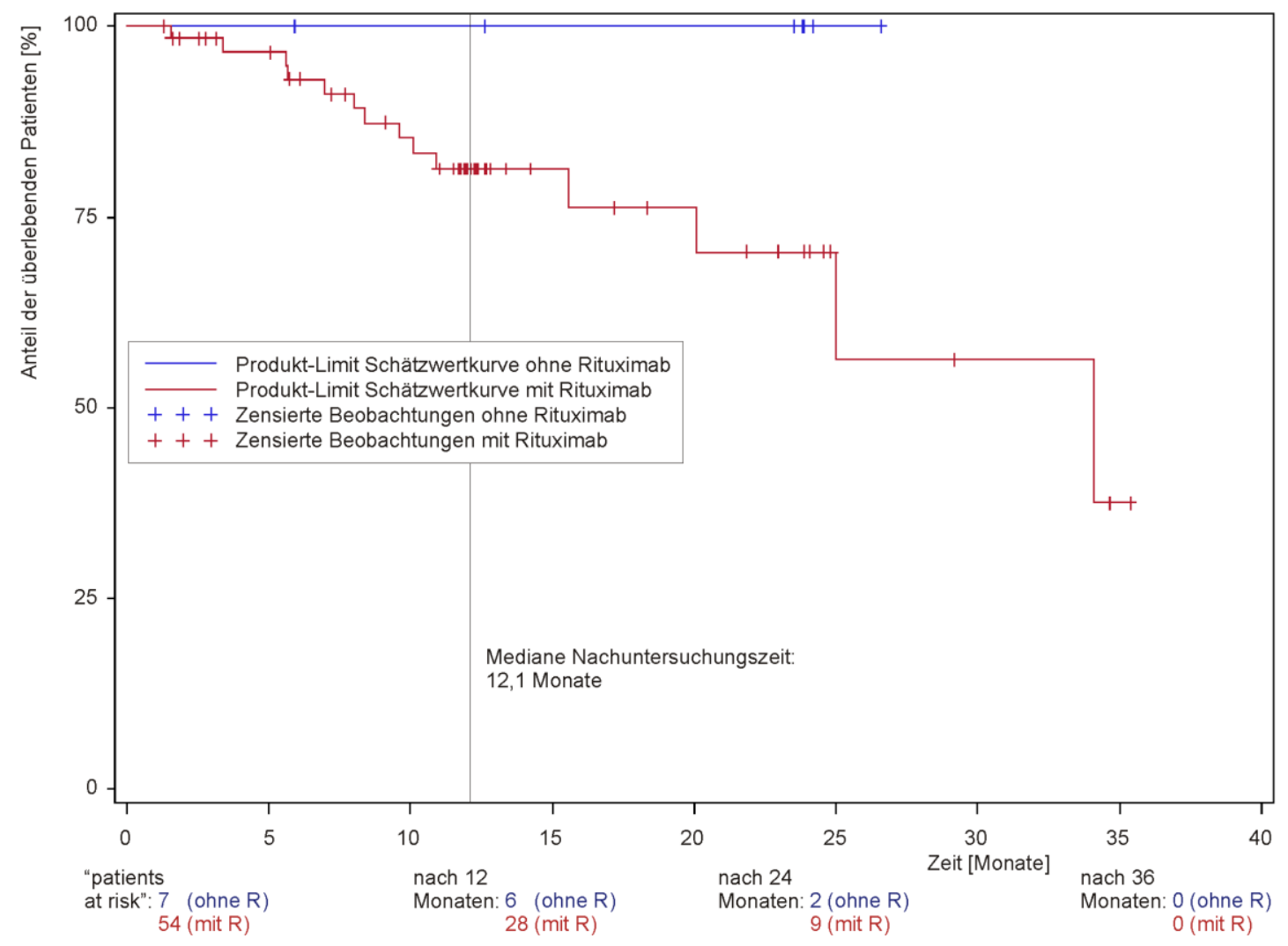

Abbildung 3.9: Gesamtüberleben (OS) der "First line"-Patienten in Abhängigkeit von Rituximab als Bestandteil der Vortherapie. 


\subsubsection{Progressfreie Zeit}

Auch die progressfreie Zeit (TTP), also die Zeitdauer bis zum erneuten Fortschreiten des Lymphoms, wurde für das gesamte Patientenkollektiv, die "First line"- und "Relapse"Patientengruppen im Vergleich und die "First line"-Subgruppen in Bezug auf Patientenalter, IPI und Rituximab in der Vortherapie bestimmt, untersucht und mittels Kaplan-MeierÜberlebenskurven dargestellt (siehe Abbildung 3.10 bis Abbildung 3.14).

Somit zeigten sich bei anfangs 181 (84,2\%) untersuchten Patienten des Gesamtkollektivs 55 Progresse im Laufe der Nachuntersuchungszeit und eine 3-Jahres-TTP von 61,0\% $(\mathrm{KI}=47,1-72,4 \%)$. Auch hier sank die Anzahl der nichtzensierten Patienten ohne Progress ("patients at risk") stark ab, sodass nach 36 Monaten nur noch vier Patienten $(1,9 \%)$ dokumentiert wurden. Es ist auffällig, dass ein Großteil der Progresse bereits kurz nach Applikation der RIT aufgetreten waren (1-Jahres-TTP = 70,0 \%; KI = 62,7-76,4 \%), während im weiteren Verlauf nur wenige weitere Ereignisse dokumentiert wurden und die Überlebenskurve ein Plateau erreicht (siehe Abbildung 3.10). Die mediane TTP, welche den Zeitpunkt beschreibt an dem die Hälfte dieser Patienten einen Progress erlitten hatten, konnte hierbei jedoch nicht genau benannt werden, da das 50,0\%-Quantil der progressfreien Zeit nach rund drei Jahren Nachuntersuchungszeit noch nicht unterschritten wurde und somit auf größer als 36 Monate geschätzt wurde. 


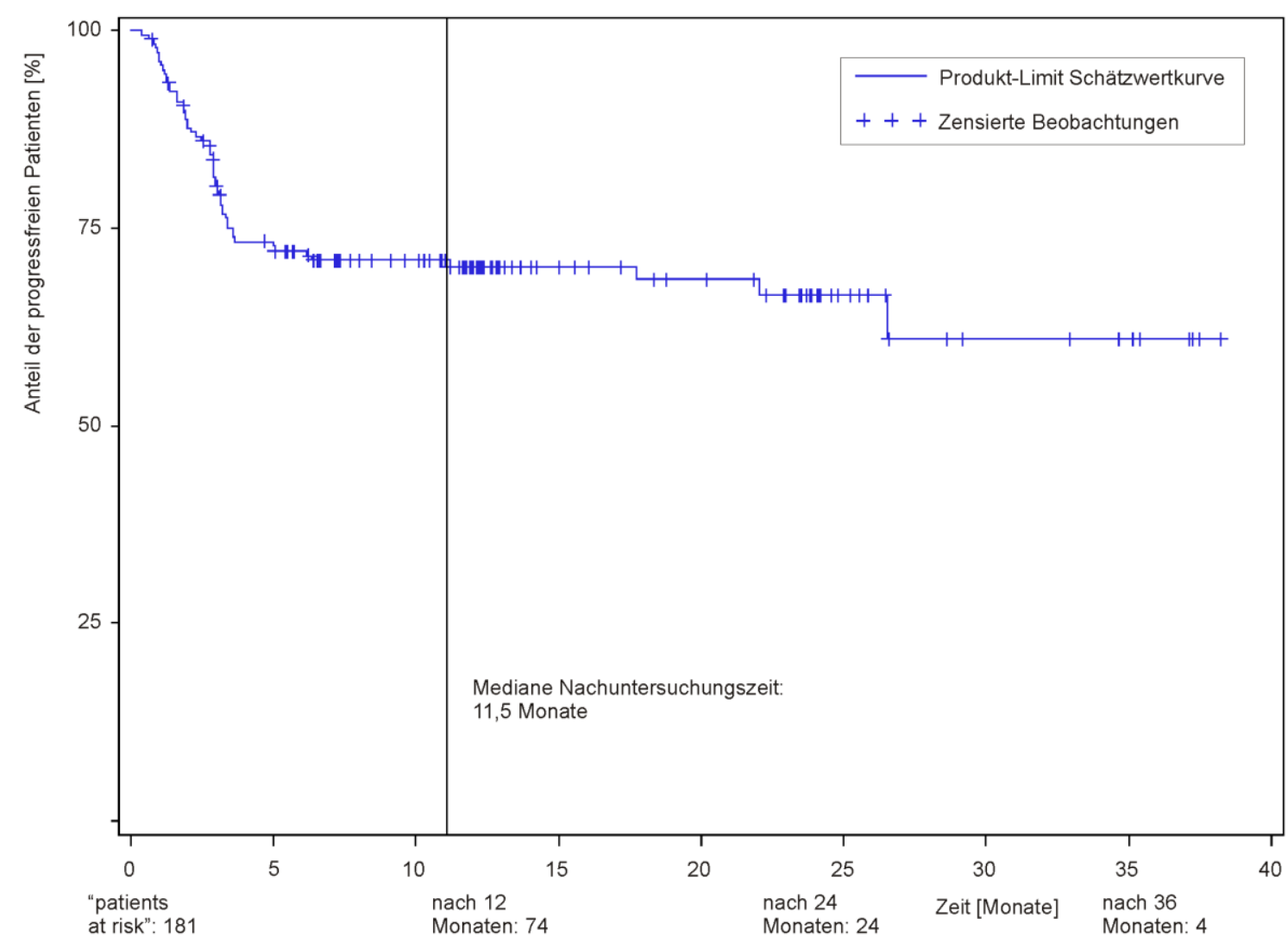

Abbildung 3.10: Progressfreie Zeit (TTP) des Gesamtkollektivs.

In der Kaplan-Meier-Analyse zum Vergleich der TTP von "First line"- und "Relapse"Patienten konnte gezeigt werden, dass die "First line"-Patienten eine signifikant höhere Wahrscheinlichkeit für Progressfreiheit aufwiesen ( $p=0,0056$ im log-Rank-Test). Es wurden neun Progresse bei anfangs 69 (95,8\%) "First line"-Patienten und 26 Progresse bei 78 $(54,4 \%)$ "Relapse"-Patienten beschrieben, so dass sich eine 3-Jahres-TTP von 87,0\% $(\mathrm{KI}=75,9-92,8)$ beziehungsweise 57,0 \% $(\mathrm{KI}=35,9-74,2)$ zeigte. Tabelle 3.10 fasst die TTPRaten nach einem, zwei und drei Nachbeobachtungsjahren für das Gesamtkollektiv, sowie die "First line"- und "Relapse"-Patientenkollektive zusammen. 


\begin{tabular}{|l|c|c|c|}
\hline & $\begin{array}{c}\text { 1-Jahres-TTP } \\
(\mathbf{K I}) \\
{[\%]}\end{array}$ & $\begin{array}{c}\text { 2-Jahres-TTP } \\
(\mathbf{K I}) \\
{[\%]}\end{array}$ & $\begin{array}{c}\text { 3-Jahres-TTP } \\
(\mathbf{K I}) \\
{[\%]}\end{array}$ \\
\hline Gesamtkollektiv & $\begin{array}{c}70,0 \\
(62,7-76,4)\end{array}$ & $\begin{array}{c}67,0 \\
(57,9-74,0)\end{array}$ & $\begin{array}{c}61,0 \\
(47,1-72,4)\end{array}$ \\
\hline "First line"-Patienten & $\begin{array}{c}87,0 \\
(75,9-92,8)\end{array}$ & $\begin{array}{c}87,0 \\
(75,9-92,8)\end{array}$ & $\begin{array}{c}87,0 \\
(75,9-92,8)\end{array}$ \\
\hline "Relapse"-Patienten & $\begin{array}{c}67,0 \\
(55,3-76,4)\end{array}$ & $\begin{array}{c}67,0 \\
(55,3-76,4)\end{array}$ & $\begin{array}{c}57,0 \\
(35,9-74,2)\end{array}$ \\
\hline
\end{tabular}

Tabelle 3.10: Anteil progressfreier Patienten nach einem, zwei und drei Jahren nach RIT für das Gesamtkollektiv, sowie die Patientengruppen "First line" und "Relapse".

Es zeigt sich, dass sich die Überlebenskurven der "First line"- und "Relapse"-Patienten früh separieren und fortan weitestgehend parallel entlang des erreichten Plateaus verlaufen (siehe Abbildung 3.11). Allerdings trat bei den "Relapse"-Patienten nach ungefähr 800 Tagen noch ein weiterer Progress auf, während bei den "First line"-Patienten nach Tag 100 kein Ereignis mehr auftrat. Die mediane TTP wurde auch hier jeweils noch nicht erreicht, so dass sie erneut auf größer als 36 Monate geschätzt werden kann. 


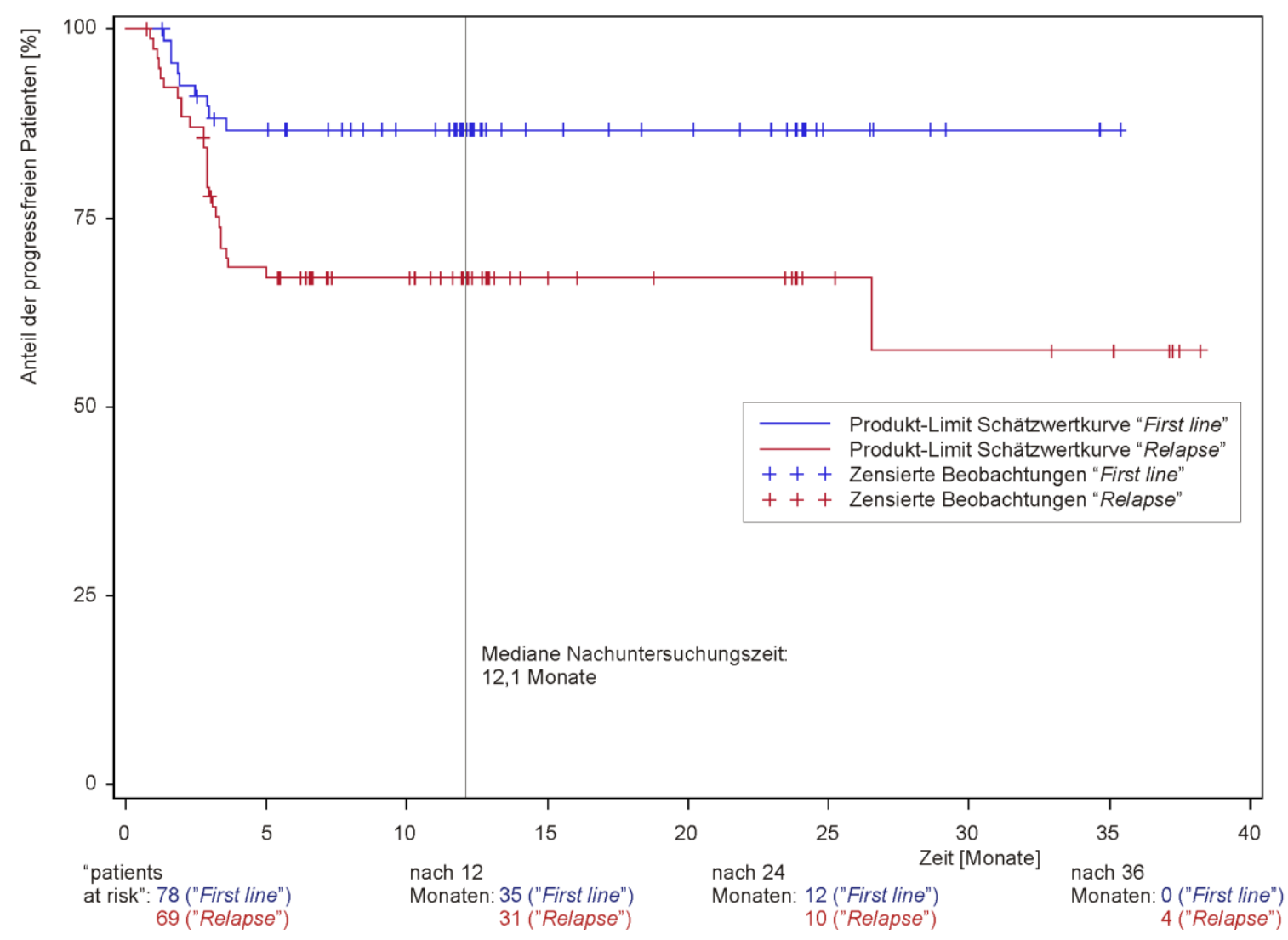

Abbildung 3.11: Progressfreie Zeit (TTP) der "First line"- und "Relapse"-Patienten im Vergleich.

Der Subgruppenvergleich beim "First line"-Kollektiv bezüglich der TTP in Abhängigkeit des Patientenalters ergab dagegen keinen signifikanten Unterschied ( $\mathrm{p}$-Wert $=0,54$ im log-RankTest) für ursprünglich 22 untersuchte Patienten (30,6\%), die jünger als 60 Jahre waren und 47 untersuchte Patienten $(69,4 \%)$, welche 60 Jahre oder älter waren. Es hatten zwei Patienten der unter 60-Jährigen und sieben Patienten der 60-Jährigen und älter einen Progress ihrer DLBCL erlitten, welcher jeweils im ersten Nachuntersuchungsjahr aufgetreten war. Im zweiten und dritten Jahr war bei keinem weiteren Patienten ein Progress dokumentiert, so dass sich eine 1-, 2- und 3-Jahres-TTP von 90,0 \% der jüngeren und von 85,0\% der älteren Patienten ergab (siehe Tabelle 3.11). Eine mediane TTP wurde jedoch auch hierbei nicht unterschritten und liegt somit bei mindestens 36 Monaten. 


\begin{tabular}{|c|c|c|c|c|c|}
\hline "First line"-Patienten & $\begin{array}{c}\text { 1-Jahres-TTP (KI) } \\
{[\%]}\end{array}$ & 2-Jah & $\begin{array}{l}\text { es-TTP (KI) } \\
{[\%]}\end{array}$ & \multicolumn{2}{|c|}{$\begin{array}{c}\text { 3-Jahres-TTP (KI) } \\
{[\%]}\end{array}$} \\
\hline < 60 Jahre & $90,0 \quad(65,3-97,4)$ & 90,0 & $(65,3-97,4)$ & 90,0 & $(65,3-97,4)$ \\
\hline$\geq 60$ Jahre & $(71,3-92,6)$ & 85,0 & $(71,3-92,6)$ & 85,0 & $(71,3-92,6)$ \\
\hline IPI $<2$ & $92,0 \quad(53,9-98,8)$ & 92,0 & $(53,9-98,8)$ & 92,0 & $(53,9-98,8)$ \\
\hline $\mathrm{IPI} \geq 2$ & $(70,7-92,4)$ & 85,0 & $(70,7-92,4)$ & 85,0 & $(70,7-92,4)$ \\
\hline mit Rituximab & $87,0 \quad(33,4-97,9)$ & 87,0 & $(33,4-97,9)$ & 87,0 & $(33,4-97,9)$ \\
\hline ohne Rituximab & $(74,9-93,0)$ & 86,0 & $(74,9-93,0)$ & 86,0 & $(74,9-93,0)$ \\
\hline
\end{tabular}

Tabelle 3.11: Anteil progressfreier Patienten nach einem, zwei und drei Jahren nach RIT für das "First line"-Patientenkollektiv in Abhängigkeit von Patientenalter, IPI und Rituximab als Therapiebestandteil.

Anhand der Kurvenverläufe nach Kaplan-Meier lässt sich der frühe Progress einiger therapierefraktären Patienten und daraufhin eine Plateaubildung ohne weitere Ereignisse in beiden Gruppen erkennen (siehe Abbildung 3.12). Die jüngeren Patienten (blaue Kurve in Abbildung 3.12) weisen dabei eine nicht signifikant geringere Wahrscheinlichkeit auf, einen Progress zu erleiden.

Ebenso ergab auch die Untersuchung der TTP in Abhängigkeit des Risikoprofils der Patienten keinen signifikanten Unterschied beim log-Rank-Test (p-Wert $=0,54)$. Von anfangs zwölf analysierten Patienten $(16,7 \%)$ mit einem IPI von kleiner zwei hatte ein Patient einen Progress im ersten Jahr, während danach kein Ereignis mehr auftrat (1-, 2- und 3-Jahres$\mathrm{TTP}=92,0 \%$; KI = 53,9-98,8\%). Vergleichend dazu war bei zu Beginn 46 analysierten Patienten $(63,9 \%)$ mit zwei oder mehr Risikofaktoren bei sieben Patienten ein Progress aufgetreten. Auch hier waren die einzigen Ereignisse im ersten Nachuntersuchungsjahr beschrieben, so dass die 3-Jahres-TTP von 85,0 \% (KI = 70,7-92,4 \%) der des ersten Jahres entspricht (siehe Tabelle 3.11). Wie schon zuvor konnte hierbei keine mediane TTP beschrieben werden.

Die Form der Überlebenskurven zeigt erneut die früh eingetretenen Progresse mit darauf folgender Plateaubildung, wobei die Patienten mit geringerem Risiko (blaue Kurve) auch eine geringere Wahrscheinlichkeit aufweisen, einen Progress zu erleiden (siehe Abbildung 3.13). 
In der Kaplan-Meier-Analyse der "First line"-Patienten in Abhängigkeit von Rituximab als Bestandteil der Vortherapie wurden anfänglich 61 Patienten $(84,7 \%)$ mit Rituximab als Bestandteil ihres Therapieprotokolls und sieben Patienten (9,7\%) ohne vorheriger Rituximabgabe untersucht. Es erlitten acht der Rituximabpatienten und einer der Patienten ohne Rituximab einen Progress im ersten Jahr. Wiederum zeigten sich keine weiteren Ereignisse im Verlauf, so dass die 1-, 2- und 3-Jahres-TTP bei 87,0 \% (KI=74,9-93,0 \%) beziehungsweise 86,0\% (KI =33,4-97,9\%) liegt und somit auch hier kein Median gebildet werden konnte (siehe Tabelle 3.11). Der Vergleich dieser beiden Gruppen bezüglich eines Unterschieds der TTP mittels des log-Rank-Tests ergab mit einem $\mathrm{p}$-Wert $=0,99$ keine Signifikanz, welches ebenfalls in den nahezu deckungsgleichen Kurvenverläufen deutlich wird (siehe Abbildung 3.14).

Wie schon zuvor in der Subgruppenanalyse des Gesamtüberlebens, standen auch hier nach 36 Monaten jeweils keine nichtzensierten progressfreien Patienten mehr zur Verfügung.

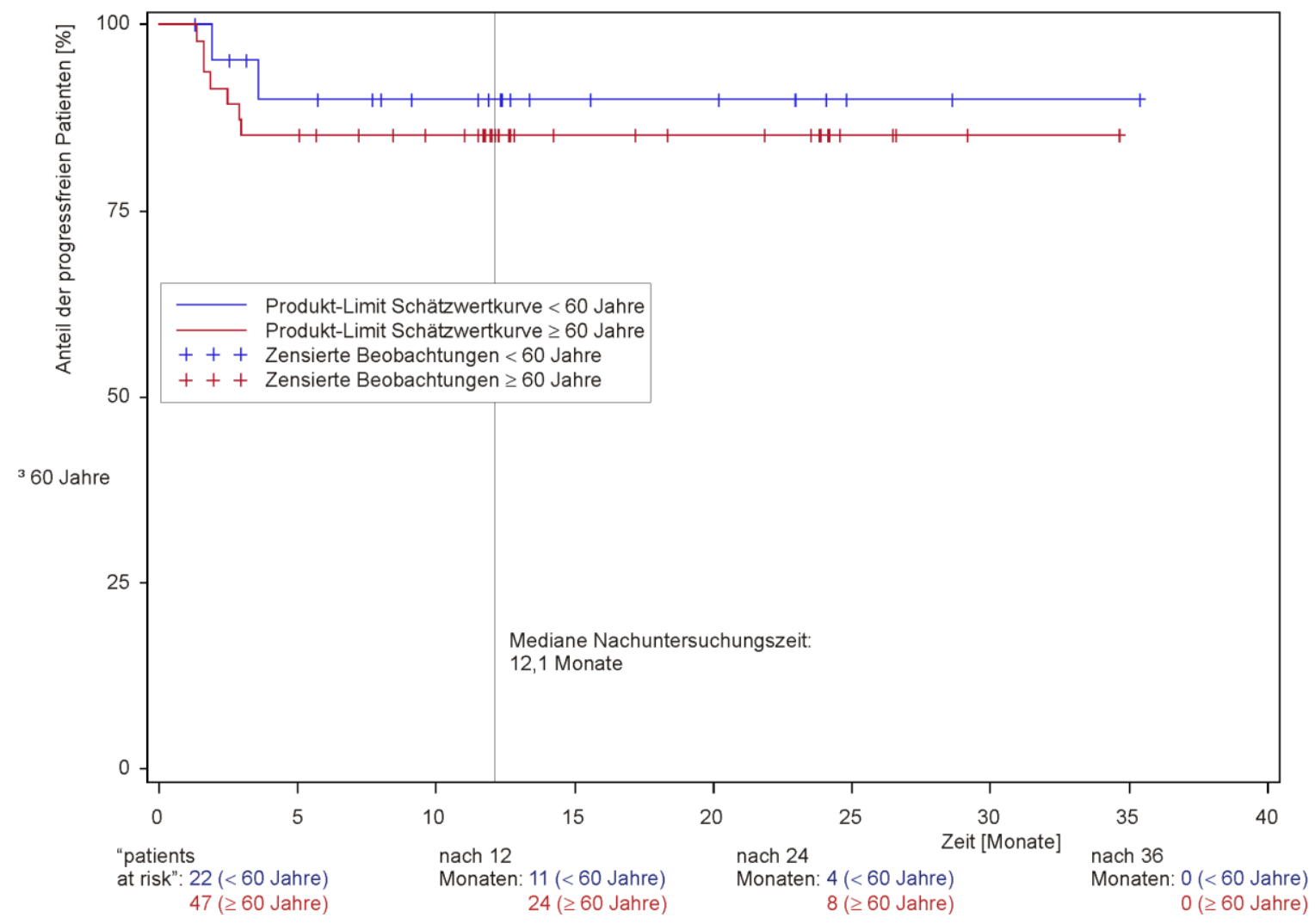

Abbildung 3.12: Progressfreie Zeit (TTP) der "First line"-Patienten in Abhängigkeit des Patientenalters. 


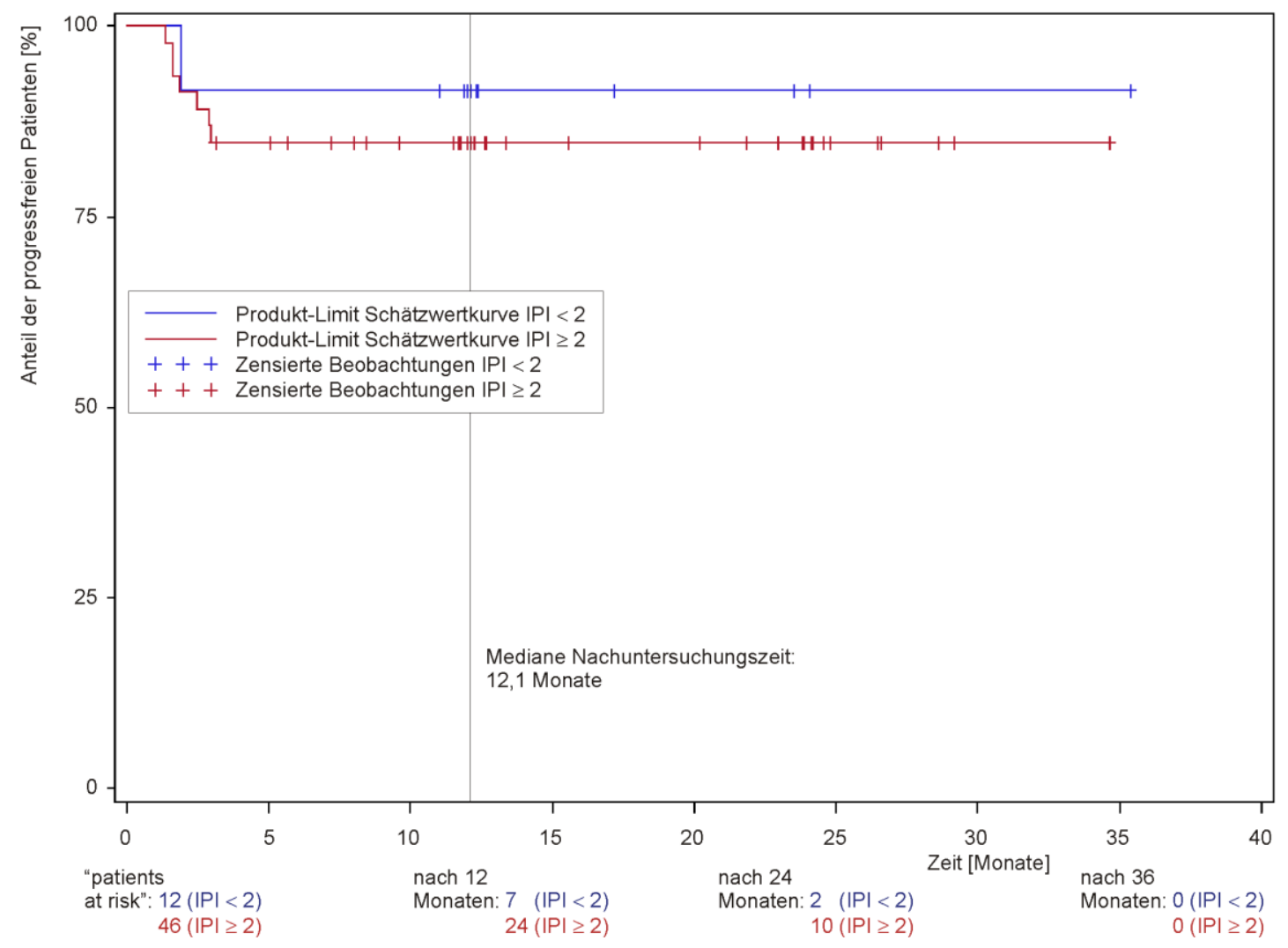

Abbildung 3.13: Progressfreie Zeit (TTP) der "First line"-Patienten in Abhängigkeit des IPI.

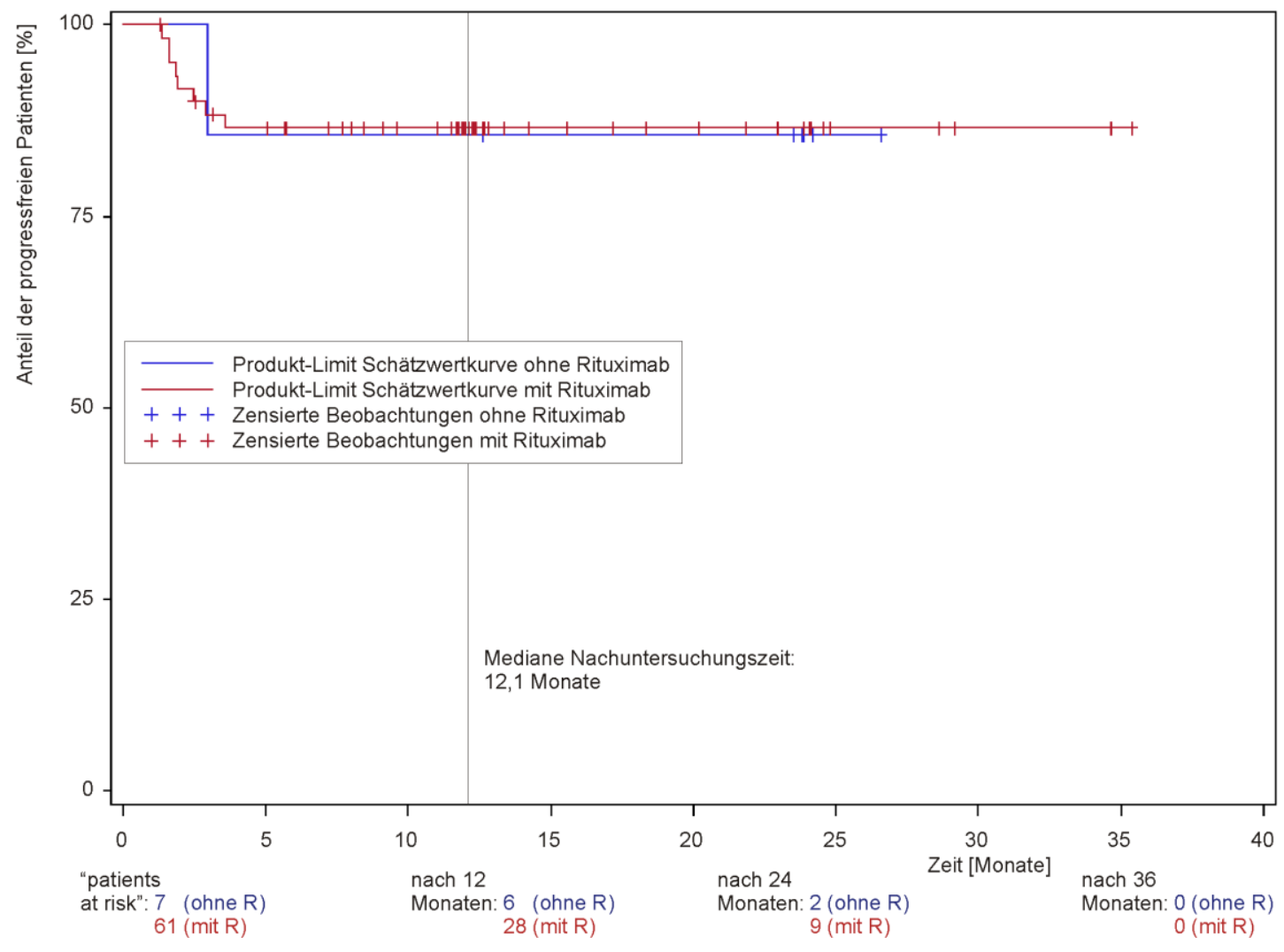

Abbildung 3.14: Progressfreie Zeit (TTP) der "First line"-Patienten in Abhängigkeit von Rituximab als Bestandteil der Vortherapie. 


\subsubsection{Progressfreies Überleben}

Neben OS und TTP wurde schließlich auch das progressfreie Überleben (PFS), also das Zeitintervall bis zum Eintreten des Todes jeglicher Art eines Patienten oder bis zum Eintreten eines Progresses des Lymphoms, beschrieben, analysiert und graphisch dargestellt (siehe Abbildung 3.15). Hierbei wurde jedoch lediglich das Gesamtkollektiv sowie die "First line"und "Relapse"-Patienten im Vergleich untersucht und keine Subgruppenanalyse der "First line"-Patienten durchgeführt.

Ursprünglich 202 Patienten (94,0\%) des Gesamtkollektivs mit 88 Todesfällen oder Progressen im Verlauf der Beobachtungen zeigten ein medianes PFS von 23,4 Monaten. Im Bezug auf die Nachuntersuchungszeit zeigten diese Patienten ein 1-Jahres-PFS von 60,0\% $(\mathrm{KI}=52,8-66,8 \%)$ bei 76 dokumentierten Progressen oder Todesfällen im ersten Jahr, ein 2-Jahres-PFS von 49,0\% $(\mathrm{KI}=40,1-57,9 \%)$ bei acht hinzugekommenen Progressen oder Todesfällen im Verlauf des zweiten Jahres sowie ein 3-Jahres-PFS von 34,0 \% (KI=20,648,3\%) bei insgesamt 88 Progressen oder Todesfällen nach drei Jahren Nachuntersuchungszeit. Auch hier lag die Anzahl nichtzensierter überlebender Patienten ohne Progress ("patients at risk") nach 36 Monaten lediglich bei vier $(1,9 \%)$.

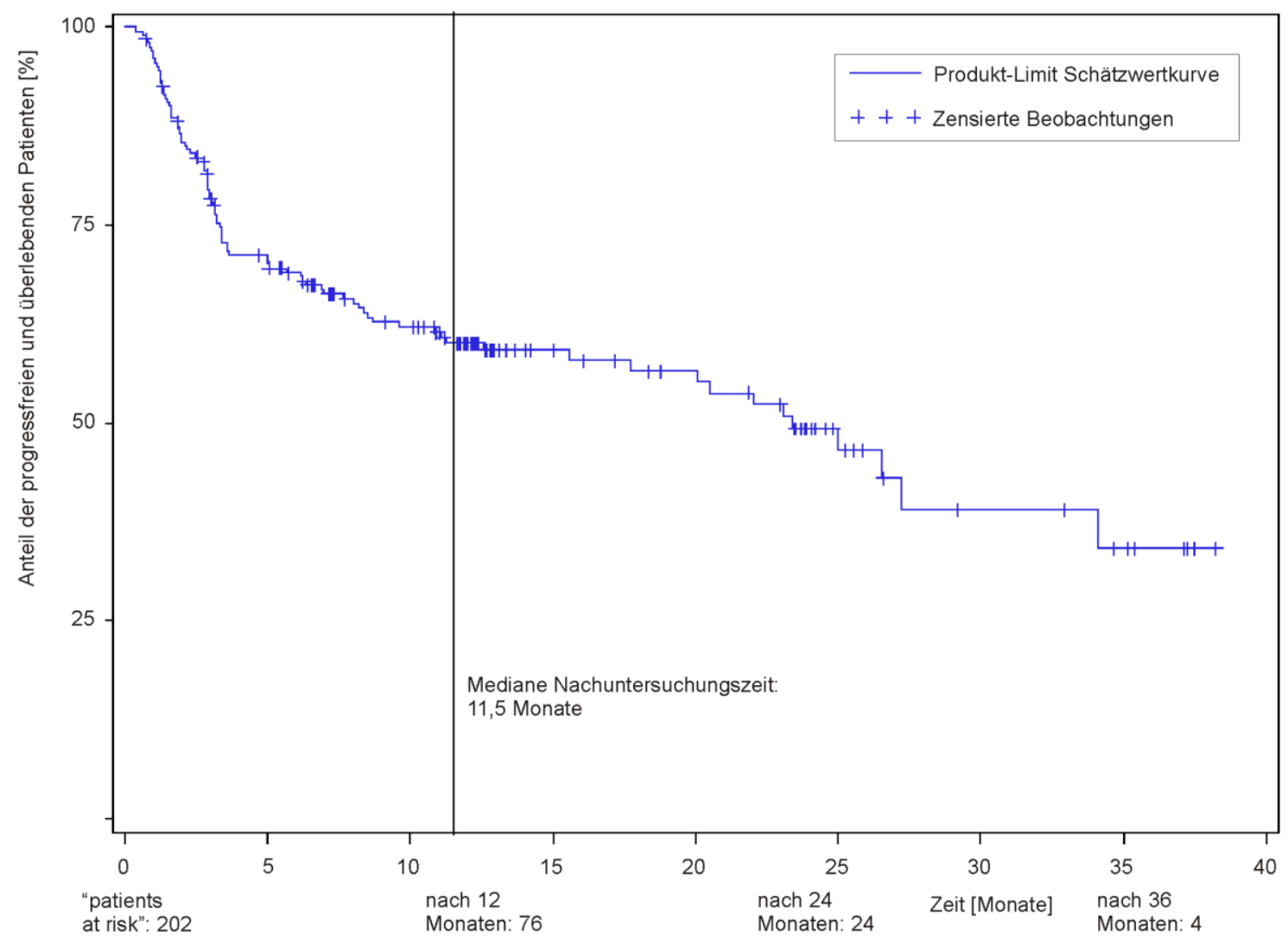

Abbildung 3.15: Progressfreies Überleben (PFS) des Gesamtkollektivs. 
Als weitere Analyse wurden zu Beginn 72 Patienten (100,0 \%) aus der "First line"-Gruppe untersucht, bei denen $21 \mathrm{mal}$ das Eintreten von Tod oder Progress dokumentiert wurde. Diese Patienten erreichten somit ein medianes PFS von 27,2 Monaten. Anfänglich 95 "Relapse"Patienten (66,4\%) mit 43 eingetretenen Ereignissen (Tod oder Progress) wiesen dagegen ein medianes PFS von nur 20,5 Monaten auf. Der Vergleich dieser beiden PFS-Kurven nach Kaplan-Meier ergab im log-Rank-Test einen $\mathrm{p}$-Wert $=0,018$, so dass ein signifikanter Unterschied der beiden Gruppen vorliegt. Im Kurvenverlauf der Kaplan-Meier-Analyse zeigt sich dementsprechend für die "First line"-Patienten eine signifikant geringere Wahrscheinlichkeit im Verlauf einen Progress zu erleiden oder zu sterben. Lediglich zum Ende des dritten Nachuntersuchungsjahres überkreuzen sich die Überlebenskurven, wobei hier jedoch nur noch wenige bis gar keine "patients at risk" zur Verfügung standen (siehe Abbildung 3.16).

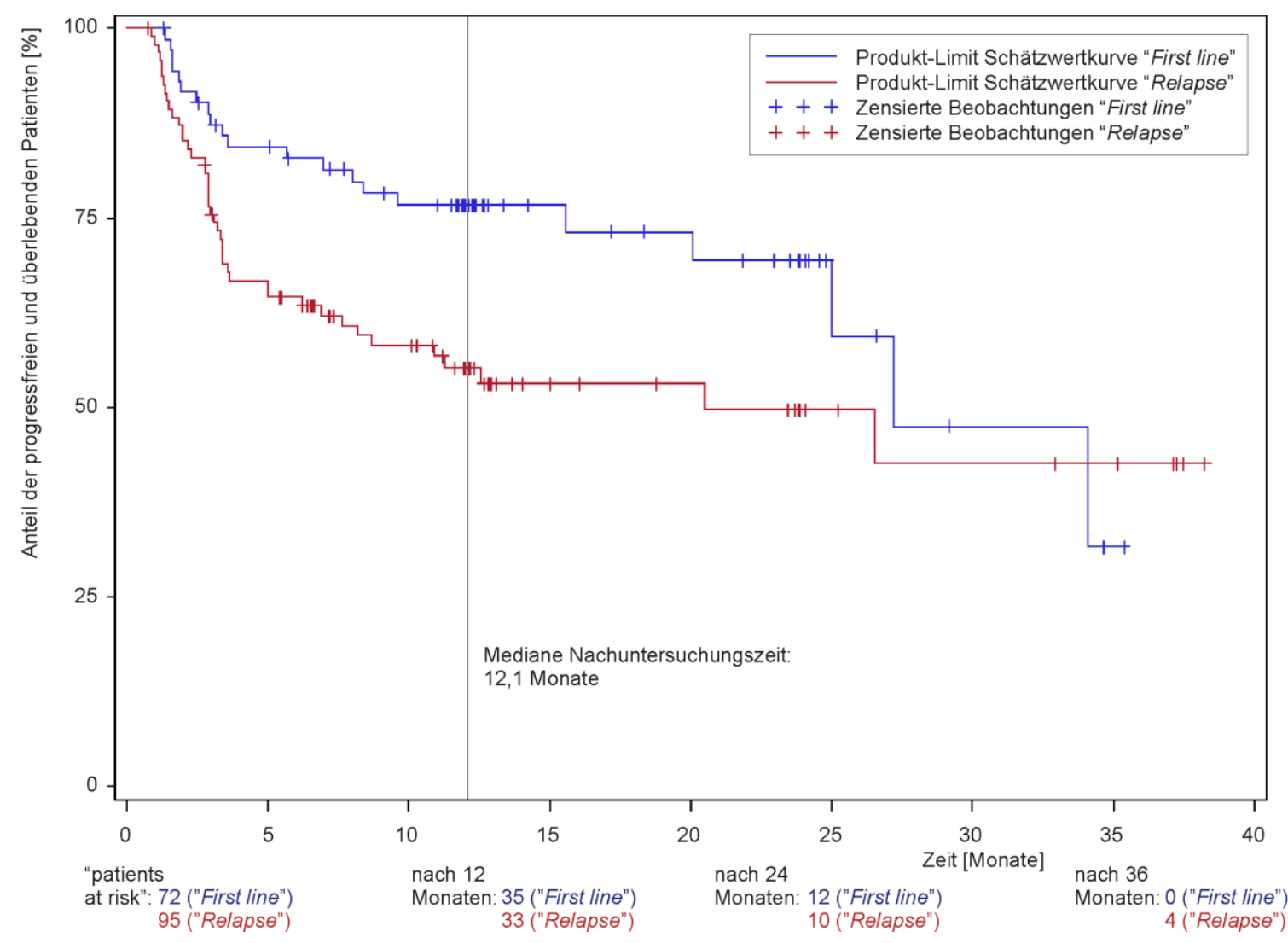

Abbildung 3.16: Progressfreies Überleben (PFS) der "First line"- und "Relapse"-Patienten im Vergleich. 
In Übereinstimmung mit dieser Beobachtung hatten nach dem ersten Jahr nach RIT 77,0\% $(\mathrm{KI}=64,7-85,0)$ der hier untersuchten "First line"-Patienten und 55,0 \% (KI = 44,1-65,0\%) der "Relapse"-Patienten progressfrei überlebt, während das 3-Jahres-PFS der "First line"Patienten mit lediglich 32,0\% $(\mathrm{KI}=6,7-61,3 \%)$ geringer als das der "Relapse"Patientengruppe (3-Jahres-PFS $=43,0 \%$ KI = 26,0-58,2 \%) ist. Das 1-, 2- und 3-Jahres-PFS des Gesamtkollektivs, der "First line"- und der "Relapse"-Patienten ist in Tabelle 3.12 tabellarisch zusammengefasst.

\begin{tabular}{|l|c|c|c|}
\hline & $\begin{array}{c}\text { 1-Jahres-PFS } \\
(\mathbf{K I}) \\
{[\%]}\end{array}$ & $\begin{array}{c}\text { 2-Jahres-PFS } \\
(\mathbf{K I}) \\
{[\%]}\end{array}$ & $\begin{array}{c}\text { 3-Jahres-PFS } \\
(\mathbf{K I}) \\
{[\%]}\end{array}$ \\
\hline Gesamtkollektiv & $\begin{array}{c}60,0 \\
(52,8-66,8)\end{array}$ & $\begin{array}{c}49,0 \\
(40,1-57,9)\end{array}$ & $\begin{array}{c}34,0 \\
(20,6-48,3)\end{array}$ \\
\hline "First line"-Patienten & $\begin{array}{c}77,0 \\
(64,7-85,0)\end{array}$ & $\begin{array}{c}69,0 \\
(53,9-80,5)\end{array}$ & $\begin{array}{c}32,0 \\
(6,7-61,3)\end{array}$ \\
\hline "Relapse"-Patienten & $\begin{array}{c}55,0 \\
(44,1-65,0)\end{array}$ & $\begin{array}{c}50,0 \\
(37,0-61,0)\end{array}$ & $\begin{array}{c}43,0 \\
(26,0-58,2)\end{array}$ \\
\hline
\end{tabular}

Tabelle 3.12: Progressfreies Überleben nach einem, zwei und drei Jahren nach RIT für das Gesamtkollektiv, sowie die Patientengruppen "First line" und "Relapse".

\subsection{Unerwünschte Nebenwirkungen der Radioimmuntherapie}

Als weitere Bestandteile der Verlaufsbeurteilung wurden unerwünschte Arzneimittelwirkungen während und nach der RIT mit ${ }^{90} \mathrm{Y}$-IT in die elektronischen Erhebungsbögen eingetragen. Da Hämatotoxizität und Myelosuppression die zu erwartenden und auch am häufigsten aufgetretenen unerwünschten Nebenwirkungen waren, wurde der Nadir folgender Laborparameter regelmäßig im Blutbild dokumentiert: Neutrophile Granulozyten, Leukozyten, Thrombozyten und Hämoglobin.

Es zeigte sich, insbesondere bei der Untersuchung der Neutrophilen Granulozyten von 128 Patienten (59,5\%), eine deutliche maximale Verminderung der Anzahl, welche im Median

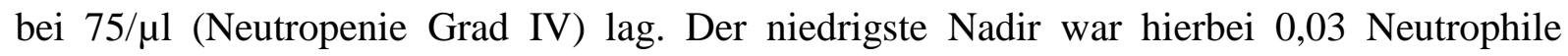


Granulozyten/ $\mu 1$, der höchste 22.440/ $\mu 1$. Bis zum Erreichen dieser Nadire seit Therapiebeginn waren im Median 33,5 Tage vergangen mit einer Verteilung von sechs bis 145 Tagen. Für 26

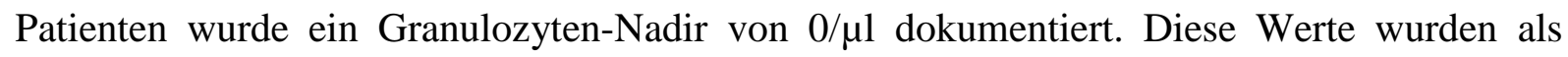
unrealistisch niedrig gewertet und auf Grund einer wahrscheinlichen Fehleingabe in die elektronischen Erhebungsbögen nicht in diese Auswertung miteinbezogen.

Die bei 142 Patienten (66,0\%) untersuchten Leukozyten im Blut zeigten einen medianen

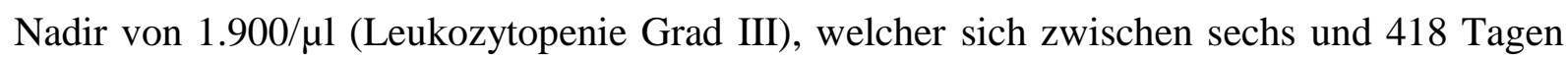
und im Mittel nach 33,5 Tagen entwickelt hatte. Der niedrigste beschriebene Nadir lag bei 10

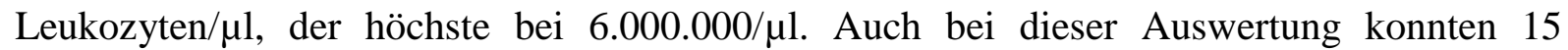
Patienten nicht eingeschlossen werden, da bei ihnen 0/ $\mu$ l als Leukozyten-Nadir angegeben worden war und von einer Fehleingabe ausgegangen werden muss.

Auch der Thrombozyten-Nadir, welcher für 178 Patienten $(82,8 \%)$ bestimmt wurde, war mit

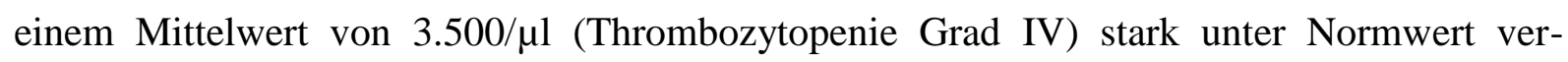
mindert. Die Anzahl variierte dabei zwischen 0,01 bis 189.000 Thrombozyten/ $\mu$ l. Hierbei wurde nach durchschnittlich 33,0 Tagen und einer Verteilung zwischen sieben bis 418 Tagen die maximale Verminderung erreicht.

Der maximal verminderte Hämoglobin-Gehalt im Blut von 178 Patienten $(82,8 \%)$ lag in einem Bereich von 5,5-14,1 g/dl, wobei der Durchschnittsgehalt bei 9,4 g/dl (Anämie Grad II) lag. Dieser zeigte sich nach einer durchschnittlichen Dauer von 40,0 Tagen nach Therapiebeginn mit einer Zeitspanne von zwei bis 418 Tagen.

Für eine komplette Erholung des Blutbildes zu normalen Laborparametern benötigten 117 dokumentierte Patienten (54,4\%) im Median 82,0 Tage. Die zeitliche Verteilung wurde dabei zwischen 22 und maximal 599 Tagen angegeben.

In Tabelle 3.13 sind diese Ergebnisse bezüglich der Hämatotoxizität und Myelosuppression der RIT zusammengefasst und mit, im RIT-Register als physiologisch gewerteten, Referenzbereichen für Erwachsene verglichen. 


\begin{tabular}{|c|c|c|c|c|c|}
\hline & $\begin{array}{c}\text { Anzahl } \\
\text { Patienten } \\
{[\mathrm{n} ; \%]}\end{array}$ & $\begin{array}{c}\text { Mediane } \\
\text { Zeit } \\
\text { [Tage] }\end{array}$ & $\begin{array}{l}\text { Medianer } \\
\text { Nadir }\end{array}$ & $\begin{array}{c}\text { Minimaler } \\
- \\
\text { Maximaler } \\
\text { Nadir }\end{array}$ & $\begin{array}{c}\text { Referenz- } \\
\text { bereich }\end{array}$ \\
\hline $\begin{array}{c}\text { Hämoglobin } \\
\text { [g/dl] }\end{array}$ & $178 ; 82,8$ & 40,0 & 9,4 & $5,5-14,1$ & $\begin{array}{l}\text { 주 } 14-18 \\
\text { ㅇ } 12-16\end{array}$ \\
\hline $\begin{array}{c}\text { Leukozyten } \\
{[/ \mu \mathrm{l}]}\end{array}$ & $142 ; 66,0$ & 33,5 & 1.900 & $\begin{array}{c}10- \\
6.000 .000\end{array}$ & $\begin{array}{l}4.300- \\
10.000\end{array}$ \\
\hline $\begin{array}{c}\text { Neutrophile } \\
\text { Granulozyten } \\
{[/ \mu \mathrm{l}]}\end{array}$ & $128 ; 59,5$ & 33,5 & 75 & $\begin{array}{c}0,03- \\
22.440\end{array}$ & $\begin{array}{r}2.500- \\
7.500\end{array}$ \\
\hline $\begin{array}{c}\text { Thrombozyten } \\
{[/ \mu \mathrm{l}]}\end{array}$ & $178 ; 82,8$ & 33,0 & 3.500 & $\begin{array}{c}0,01- \\
189.000\end{array}$ & $\begin{array}{c}150.000- \\
300.000\end{array}$ \\
\hline
\end{tabular}

Tabelle 3.13: maximal verminderte Anzahl von Hämoglobin, Leukozyten, Neutrophilen Granulozyten und Thrombozyten im Blutbild nach RIT im Vergleich mit klinischen Referenzwerten.

Weitere unerwünschte Ereignisse, die während oder nach der RIT auftraten, wurden für 14 Patienten $(6,5 \%)$ dokumentiert. Hierbei trat jedes Ereignis jedoch nur einmalig auf und es zeigte sich keine signifikante Häufung bestimmter Symptome oder Krankheitsbilder. Am häufigsten waren dermatologische Erscheinungen wie Bläschen im zentralen Gebiet der tumorinfiltrierten Haut, ein Erysipel der Leiste, ein Lymphknotenabszess sowie eine Entzündung der Hand aufgetreten. Beschriebene Symptome des Gastrointestinaltrakts reichten von einer milden Diarrhoe über eine Cholezystitis bis hin zu einer gastrointestinalen Massenblutung. Es zeigte sich eine Beeinträchtigung des respiratorischen Systems durch eine dokumentierte Infektion der oberen Atemwege im Zusammenhang mit der RIT, sowie eine segmentale Lungenembolie, die als Zufallsbefund bei einer computertomographischen Untersuchung während der RIT diagnostiziert wurde. Des Weiteren erkrankte ein Patient an einer Niereninsuffizienz und ein anderer litt unter Hyperurikämie. Hierbei ist jedoch unklar, ob diese auf Grund gesteigerter Harnsäurebildung im Rahmen der Behandlung oder durch eine verminderte renale Ausscheidung zu begründen ist. Im Rahmen der Immunsuppression war bei einem Patienten eine Reaktivierung einer vergangenen Zytomegalie-Virusinfektion 
aufgetreten, sowie, wahrscheinlich im Zusammenhang mit einer intensivmedizinischen Behandlung, eine Critical-Illness-Polyneuropathie diagnostiziert worden. Außerdem wurden psychosomatische Symptome wie Halluzinationen und Angstgefühl, wie auch Allgemeinsymptome, nämlich eine leichte Asthenie, beschrieben. Bei einigen Patienten $(n=3 ; 1,4 \%)$ waren mehrere dieser unerwünschten Ereignisse aufgetreten, für die restlichen $(\mathrm{n}=11 ; 5,1 \%)$ war jedoch jeweils nur eine dieser unerwünschten Arzneimittelwirkungen beschrieben.

Bei einem Patienten trat ein myelodysplastisches Syndrom (MDS) in Folge der RIT auf und bei einem weiteren Patienten wurde der Verdacht auf ein MDS dokumentiert. Als sekundäre Neoplasien sind ein pleomorphes Lungenkarzinom, eine Transformation des DLBCL in ein NK-/T-Zell-Lymphom sowie das Auftreten eines weiteren, nicht näher bezeichneten, soliden Tumors nach der RIT beschrieben. Somit war bei 2,3\% $(n=5)$ der mit RIT behandelten Patienten eine sekundäre, maligne Erkrankung und bei 0,9\% $(n=2)$ ein MDS aufgetreten.

\subsubsection{Unerwünschte Arzneimittelwirkung bei "First line"-Patienten}

Im Folgenden soll, als Marker für eine hämatotoxische beziehungsweise myelosuppressive Arzneimittelwirkung der RIT, die maximal verminderte Anzahl des Hämoglobin-Gehalts, der Leukozyten, der Neutrophilen Granulozyten und der Thrombozyten, welche sich auch im Blutbild des "First line"-Patientenkollektivs zeigte, beschrieben werden.

Von $72(100,0 \%)$ dieser Gruppe zugehörigen Patienten wurde für 60 Patienten $(83,3 \%)$ der Hämoglobin-Nadir, für 65 Patienten $(90,3 \%)$ der Nadir der Neutrophilen Granulozyten, für 66 Patienten $(91,7 \%)$ der Thrombozyten-Nadir und für 59 Patienten $(82,0 \%)$ der Leukozyten-Nadir dokumentiert.

Es zeigte sich hierbei ein maximal erniedrigter Hämoglobin-Gehalt von durchschnittlich 10,0 g/dl (Anämie Grad I) mit einer Variationsbreite von 5,5-14,0 g/dl. Zur Ausbildung dieser Nadire waren zwischen elf und 91 Tage seit der RIT vergangen, der Median lag bei 42,5 Tagen.

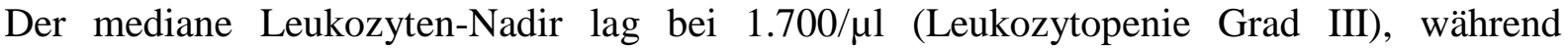
insgesamt Werte in einem Bereich von 100-4.300.000 Leukozyten/ $\mu 1$ aufgezeichnet wurden. Nach frühestens sechs Tagen, spätestens nach 69 Tagen und im Median nach 35,5 Tagen waren diese Tiefstwerte aufgetreten.

Die Anzahl Neutrophiler Granulozyten war nach einem Zeitraum von acht bis 69 Tagen nach

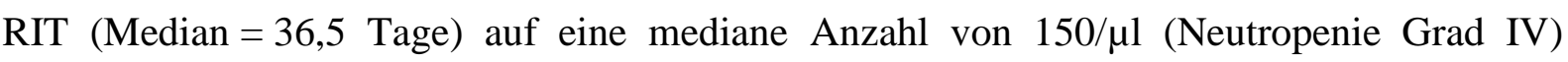


deutlich vermindert. Der Wertebereich lag hierbei zwischen 0,2-2.800 Neutrophilen Granulozyten/ $\mu 1$.

Die Thrombozyten-Nadire, welche nach durchschnittlich 33,0 Tagen und in einem Zeitraum zwischen 15 bis 63 Tagen aufgetreten waren, erreichten eine maximal verminderte Anzahl von 5-140.000 Thrombozyten/ $\mu$ l. Der durchschnittliche Nadir lag bei 3.071/ $\mu 1$ (Thrombozytopenie Grad IV).

Die Zeitspanne bis zur kompletten Erholung des Blutbildes nach RIT wurde für 57 Patienten $(79,2 \%)$ dokumentiert. Es zeigte sich, dass im Median 82,0 Tage bis zum Erreichen normwertiger Blutwerte vonnöten waren, wobei die frühste Erholung nach 42 Tagen und die späteste nach 599 Tagen eintrat. Tabelle 3.14 fasst die beschriebene Hämatotoxizität und Myelosuppression für "First line"-Patienten anhand des jeweiligen Nadirs zusammen und vergleicht diese Blutwerte mit den im RIT-Register verwendeten klinischen Referenzwerten für Erwachsene.

\begin{tabular}{|c|c|c|c|c|c|}
\hline & $\begin{array}{c}\text { Anzahl } \\
\text { Patienten } \\
{[\mathbf{n} ; \%]}\end{array}$ & $\begin{array}{c}\text { Mediane } \\
\text { Zeit } \\
\text { [Tage] }\end{array}$ & $\begin{array}{l}\text { Medianer } \\
\text { Nadir }\end{array}$ & $\begin{array}{c}\text { Minimaler } \\
- \\
\text { Maximaler } \\
\text { Nadir }\end{array}$ & $\begin{array}{l}\text { Referenz- } \\
\text { bereich }\end{array}$ \\
\hline $\begin{array}{l}\text { Hämoglobin } \\
\text { (g/dl) }\end{array}$ & $60,83,3$ & 42,5 & 10,0 & $5,5-14,0$ & $\begin{array}{l}\text { o } 14-18 \\
\text { ○ } 12-16\end{array}$ \\
\hline $\begin{array}{c}\text { Leukozyten } \\
(/ \mu \mathrm{l})\end{array}$ & $59,82,0$ & 35,5 & 1.700 & $\begin{array}{c}100- \\
4.300 .00\end{array}$ & $\begin{array}{l}4.300- \\
10.000\end{array}$ \\
\hline $\begin{array}{c}\text { Neutrophile } \\
\text { Granulozyten } \\
(/ \mu \mathrm{l})\end{array}$ & $65,90,3$ & 36,5 & 150 & $0,1-2.800$ & $2.500-7.500$ \\
\hline $\begin{array}{c}\text { Thrombozyten } \\
(/ \mu \mathrm{l})\end{array}$ & $66,91,7$ & 33,0 & 3.071 & $5-140.000$ & $\begin{array}{c}150.000- \\
300.000\end{array}$ \\
\hline
\end{tabular}

Tabelle 3.14: maximal verminderte Anzahl von Hämoglobin, Leukozyten, Neutrophilen Granulozyten und Thrombozyten im Blutbild nach RIT bei "First line"-Patienten im Vergleich mit klinischen Referenzwerten. 


\section{Diskussion}

\subsection{Die Bedeutung und Aussagekraft der untersuchten Registerdaten}

In der vorliegenden Arbeit wurde mittels 215 in einem internetbasierten Register erfasster Patientendaten die Durchführbarkeit und Wirksamkeit einer RIT bei DLBCL gezeigt. Trotz der sich hierbei ergebenden Herausforderungen der Datenerfassung (siehe Kapitel 2.4, S.26), scheint durch die Größe des analysierten Patientenkollektivs die Anwendung von RIT im klinischen Alltag unselektioniert widergespiegelt zu werden.

Daten, welche wie hier aus einem internetbasierten Register stammen, haben im Vergleich zu Daten aus klinisch prospektiven Studien stets eine andersartige Aussagekraft. Dies liegt vor allem begründet in der lediglich retrospektiv deskriptiven Auswertung der Daten, der fehlenden Beeinflussungsmöglichkeit des Therapieverlaufs, der Abhängigkeit von den dokumentierenden Ärzten sowie den eingeschränkten Kontroll- und Überwachungsmöglichkeiten bei der Dateneingabe in das Register. Auch in der vorliegenden Arbeit ist dadurch die Aussagekraft und Validität der analysierten Daten limitiert. Um dennoch einen möglichst großen Informationswert aus der Analyse der Registerdaten zu bekommen, wurde ein relativ großes und heterogenes Patientenkollektiv $(n=215)$ in die Untersuchung eingeschlossen und versucht, möglichst vollständige Dokumentationen zu erhalten. Dass Letzteres nicht für sämtliche Patienten möglich war und besonders Langzeitdaten nur eingeschränkt erfasst wurden (siehe Kapitel 3.3, S.39), zeigt sich vornehmlich in der Auswertung der Überlebenskurven. Hier muss allerdings bedacht werden, dass die Applikation der RIT bei vielen Patienten zum Ende des Datenerhebungszeitraums im Dezember 2010 noch keine 24 oder sogar 36 Monate zurücklag und somit diese Daten auch noch nicht erhoben werden konnten. Bei einer erneuten Auswertung der RIT-Register-Daten in einiger Zeit würden möglicherweise mehr dokumentierte Nachuntersuchungen vorliegen und somit auch aussagekräftigere Angaben über eine Langzeitprognose der Patienten gemacht werden können.

Im Folgenden sollen die verschiedenen Auswertungen und Ergebnisse der vorliegenden Patientendaten bezüglich ihrer Übertragbarkeit auf den klinischen Alltag sowie ihrer Aussagekraft und Validität analysiert und diskutiert werden. 


\subsubsection{Analyse und Diskussion der Charakteristika des Patientenkollektivs}

Das DLBCL-Patientenkollektiv, welches vom RIT-Register erfasst wurde und dessen Daten die Grundlage der vorliegenden Analyse darstellen, repräsentiert anschaulich das durchschnittliche Patientenkollektiv in der alltäglichen Klinikroutine. Beispielsweise ist hier das Durchschnittsalter bei Erstdiagnose zu nennen, welches im Gesamtkollektiv bei 62 Jahren und im "First line"-Kollektiv bei 65 Jahren liegt und somit nahezu dem weltweit mittleren Erkrankungsalter von 64 Jahren bei DLBCL entspricht.

Allgemeine anamnestische Informationen sowie Details über den bisherigen Krankheitsverlauf, Vortherapien und die Anwendung der RIT wurden im großen Umfang dokumentiert, sodass hier von einem großen Informationswert ausgegangen werden kann. Wie weiter unten beschrieben, entstanden lediglich bei der Dokumentation des IPI und der Indikationsstellungen größere Lücken in der Datenerhebung.

So war es beispielsweise bei rund einem Drittel der Patienten $(\mathrm{n}=71,33,0 \%)$ nicht möglich, den jeweiligen IPI zu bestimmen, da die Dokumentation eines oder mehrerer Risikofaktoren fehlte. Folglich ist hier die Aussagekraft, besonders über das Risikoprofil des Gesamtkollektivs, limitiert. Von den 144 Patienten des Gesamtkollektivs (67,0\%), bei denen eine IPI-Bestimmung möglich gewesen ist, hatten 87 Patienten $(60,4 \%$, beziehungsweise 40,5\% des Gesamtkollektivs) ein niedriges bis niedrigintermediäres Risiko. Für 57 Patienten (39,6 \% derer mit IPI-Dokumentation, beziehungsweise 26,5\% des Gesamtkollektivs) musste dagegen anhand des ermittelten IPI von einem intermediär hohen bis hohen Risiko ausgegangen werden.

Erfreulicherweise liegt der Anteil unvollständig dokumentierter Risikofaktoren im "First line"-Patientenkollektiv $(\mathrm{n}=72)$ niedriger, so dass das Risikoprofil dieser Patienten sicherer bestimmt werden konnte. Eine Bestimmung des IPI war hier lediglich bei elf Patienten $(15,3 \%)$ nicht eindeutig möglich gewesen. In diesem Kollektiv zeigte sich beinahe bei der Hälfte aller Patienten $(n=34 ; 47,2 \%)$ ein niedriges oder niedrigintermediäres Risiko.

Wie sich auch im klinischen Alltag zeigt, hatte das Gesamtkollektiv der analysierten Patienten ein heterogenes $\mathrm{Ma} ß$ an vorherigen Therapielinien (eine bis sieben vorherige Therapielinien) erhalten. Entsprechend den aktuellen Leitlinien (siehe Kapitel 1.1.3.1, S.11) wurden die meisten Patienten $(\mathrm{n}=209 ; 97,2 \%)$ chemotherapeutisch vorbehandelt, wobei der Antikörper Rituximab größtenteils $(\mathrm{n}=185 ; 86,0 \%)$ ein Therapiebestandteil war. 
Trotz dieser zahlreich dokumentierten Vortherapien wurde für rund ein Fünftel der Patienten $(\mathrm{n}=44 ; 20,5 \%)$ keine Aussage zur Therapielinie der verabreichten RIT gemacht. Von den 171 Patienten $(79,5 \%)$, für welche eine Indikationsstellung bezüglich der Therapielinie vorlag, hatten insgesamt 72 Patienten $(42,1 \%$, beziehungsweise 33,5\% von 215 Patienten des Gesamtkollektivs), die RIT als Erstlinientherapie entweder zur Konsolidierung nach Chemotherapie, als primäre Therapie oder als Konditionierung im Rahmen einer HDC mit BEAM und anschließender Stammzelltransplantation erhalten. Diese Patienten waren also zuvor unbehandelt und wurden als "First line"-Patienten gesondert analysiert.

Bei den übrigen Patienten ("Relapse", $\mathrm{n}=143$ ), war die am häufigsten gestellte Indikation zur RIT ebenfalls eine Konsolidierungstherapie nach Chemotherapie. Dennoch spielten hier, wie sich auch in der Klinikroutine zeigt, Rezidive und therapierefraktäre Lymphome eine große Rolle. Insgesamt 28 rezidivierte und 21 therapierefraktäre Lymphome wurden in diesem Patientenkollektiv radioimmuntherapeutisch behandelt.

Wie bereits einleitend zur klinischen Anwendung der RIT beschrieben (siehe Kapitel 1.2.2, S.16), wird das $\mathrm{zu}$ verabreichende Radioimmuntherapeutikum ${ }^{90} \mathrm{Y}$-IT abhängig vom Körpergewicht und der Thrombozytenzahl im Blut dosiert. Bei normaler Thrombozytenanzahl liegt die infundierte Energiedosis normalerweise bei $0,4 \mathrm{mCi} / \mathrm{kg}$ Körpergewicht und wird bei einer Thrombozytopenie ersten Grades auf $0,3 \mathrm{mCi} / \mathrm{kg}$ Körpergewicht reduziert (Chapuy et al. 2007; Emmanouilides 2007). Dementsprechend liegt auch der Median der eingesetzten Energiedosis im RIT-Register bei $0,34 \mathrm{mCi} / \mathrm{kg}$ Körpergewicht im Gesamtkollektiv und bei $0,35 \mathrm{mCi} / \mathrm{kg}$ Körpergewicht im "First line"-Patientenkollektiv. Obwohl durch die Bestimmung des Medians versucht wurde, den Einfluss stark abweichender Angaben zu reduzieren, bleibt weiterhin unsicher, ob dokumentierte Dosen von beispielsweise $0,01 \mathrm{mCi} / \mathrm{kg}$ Körpergewicht oder $0,67 \mathrm{mCi} / \mathrm{kg}$ Körpergewicht tatsächlich appliziert wurden und somit unter Umständen das Nebenwirkungsprofil beeinflussen oder ob es sich hierbei um Dokumentationsfehler handelt.

Zusammenfassend zeigt sich, dass das in der vorliegenden Arbeit untersuchte, nicht selektionierte Patientenkollektiv den Patienten der alltäglichen Klinikroutine entspricht und wie diese ein heterogenes Spektrum an Krankheitsausprägungen aufweist. Patienten klinischer Studien müssen dagegen genau definierte Kriterien erfüllen und innerhalb einer Studie eine homogene Gruppe bilden. Durch diesen Unterschied resultiert, dass im Folgenden nicht immer ein genauer, direkter Vergleich zwischen Register- und Studienpatienten möglich 
ist. Es wurde jedoch versucht, möglichst ähnliche Patientenkollektive miteinander zu vergleichen.

\subsubsection{Analyse und Diskussion der klinischen Ergebnisse und unerwünschter Arzneimittelwirkungen}

Anhand der dokumentierten Ansprechraten und Langzeitergebnisse sowie der unerwünschten Arzneimittelwirkungen, die im Verlauf aufgetreten sind, zeigt sich die Sicherheit, Durchführbarkeit und Effizienz der RIT in den untersuchten Patientenkollektiven der vorliegenden Arbeit. Um die erzielten Ergebnisse besser in den Kontext aktueller Therapieleitlinien und Studien zur Anwendung von RIT bei DLBCL einordnen zu können, werden diese in den folgenden Kapiteln vergleichend diskutiert (siehe Kapitel 4.2, S.70 und Kapitel 4.3, S.81). Zuvor soll jedoch die jeweilige Aussagekraft des Therapieansprechens, der Langzeitergebnisse und der unerwünschten Arzneimittelwirkungen analysiert werden:

Das Therapieansprechen wurde für die meisten der Patienten des Gesamtkollektivs ( $\mathrm{n}=189$; $87,9 \%)$ und fast alle Patienten des "First line"-Kollektivs $(\mathrm{n}=69 ; 95,8 \%)$ beschrieben, so dass hier eine ausreichend große Aussagekraft durch die Masse der Daten gegeben ist. Wie sich in den folgenden Kapiteln zeigen wird, liegen die hier erreichten Ansprechraten in ähnlichen Bereichen, wie solche aus unabhängigen und randomisierten klinischen Studien. Somit kann hier von einer unverfälschten Wiedergabe der klinischen Anwendung der RIT und einer Übertragbarkeit der folgenden Studienergebnisse auf eine klinische Routinebehandlung ausgegangen werden. Erwartungsgemäß zeigen die "First line"-Patienten ein besseres Therapieansprechen $(\mathrm{ORR}=83,3 \%)$ als das Gesamtkollektiv $(\mathrm{ORR}=63,3 \%)$, da diese zwar ein ähnliches Risikoprofil und ein höheres medianes Alter, aber per definitionem keine multiplen Vortherapien oder mehrfach rezidivierte und therapierefraktäre Lymphome aufwiesen. Die Auswertung der vorliegenden Arbeit zeigt somit deutlich, dass die RIT in Abhängigkeit der Indikationsstellung und Therapielinie unterschiedlich wirksam ist. Da das Therapieansprechen der jüngeren (<60 Jahre) und älteren $(\geq 60$ Jahre) "First line"-Patienten ähnlich gut verteilt ist $(\mathrm{ORR}=81,8 \%$ beziehungsweise $84,0 \%)$, zeigt sich hier kein eindeutiger Vorteil in Abhängigkeit des Alters im Therapieansprechen bei Erstlinienpatienten.

Wie bereits beschrieben, zeigt sich eine Beeinträchtigung durch die im RIT-Register nicht verfügbaren Daten besonders in der Aussagekraft von Langzeitauswertungen, da anfangs noch viele und erst im Verlauf der Nachuntersuchungen immer weniger Patientendaten dokumentiert wurden (Tabelle 4.1). Dieses drückt sich beispielsweise sowohl in der recht 
kurzen medianen Nachbeobachtungszeit von knapp einem Jahr (11,5 Monate) als auch in den Kurvenverläufen der Kaplan-Meier-Analysen von OS, TTP und PFS des Gesamtkollektivs aus, welche im hinteren Teil auf Grund mangelnder Daten beziehungsweise zu geringer Anzahl an nichtzensierten lebenden oder ereignisfreien Patienten an Aussagekraft verlieren. Es zeigt sich zudem, dass die Konfidenzintervalle der Überlebensraten im Verlauf immer breiter und somit weniger aussagekräftig werden. Dadurch sind letztendlich lediglich die Langzeitergebnisse bis zu zwölf Monaten, welche ja auch annähernd der medianen Nachuntersuchungszeit (11,5 Monate im Gesamtkollektiv, 12,1 Monate bei den "First line"Patienten) entsprechen, ausreichend vergleichbar mit solchen aus klinischen Studien (siehe Kapitel 3.3.2, S.42, Kapitel 3.3.3, S.49 und Kapitel 3.3.4, S.56).

\begin{tabular}{|c|c|c|}
\hline Verlaufsbeurteilung & Anzahl [n] & Prozent [\%] \\
\hline 3 Monate & 183 & 85,1 \\
\hline 6 Monate & 157 & 73,0 \\
\hline 12 Monate & 111 & 51,6 \\
\hline 24 Monate & 43 & 20,0 \\
\hline 36 Monate & 9 & 4,1 \\
\hline
\end{tabular}

Tabelle 4.1: Übersicht der durchgeführten und dokumentierten Verlaufsbeobachtungen im Gesamtkollektiv.

Wie sich bereits bei der Analyse des Therapieansprechens zeigte, weisen die Patienten des "First line"-Kollektivs auch nach bis zu 24 Monaten den Erwartungen entsprechend bessere Ergebnisse bezüglich OS, TTP und PFS auf, als das Gesamtkollektiv und die "Relapse"Patienten (siehe Tabelle 3.8, S.45, Tabelle 3.10, S.51 und Tabelle 3.12, S.58). Lediglich nach 36 Monaten verschwindet dieser Effekt, wobei jedoch zu beachten ist, dass hier keine "patients at risk" mehr im "First line"-Kollektiv zur Verfügung standen und somit die Aussagekraft stark vermindert wird. Auch in den grafischen Analysen nach Kaplan-Meier ist das signifikant bessere OS, TTP und PFS der "First line"-Patienten im Vergleich zum "Relapse"-Kollektiv erkennbar (siehe Abbildung 3.6, S.44, Abbildung 3.11, S.52 und 
Abbildung 3.16, S.57). Erneut weisen die hinteren Kurvenverläufe einen zu geringen Informationswert auf, um darüber eine deutliche Aussage treffen zu können und diese mit Studienergebnissen $\mathrm{zu}$ vergleichen. Insgesamt präsentiert sich jedoch wieder die bereits beschriebene Abhängigkeit der RIT von der vorliegenden Indikationsstellung.

Betrachtet man nun die verschiedenen Kaplan-Meier-Analysen der "First line"-Subgruppen, so zeigt sich hier eine besonders deutliche Einbuße an Aussagekraft durch die progrediente Nichtverfügbarkeit von Patientendaten, da diese untersuchten Kollektive bereits zu Beginn der Untersuchung lediglich eine geringe Patientenanzahl besaßen. Die Subgruppen wurden in Anlehnung an die drei verschiedenen Patientengruppen der aktuellen Therapieleitlinien eingeteilt. Obwohl deutliche Unterschiede im Gesamtüberleben und der Progressfreiheit in Abhängigkeit von Alter, IPI und Rituximabgabe zu erwarten gewesen wären, zeigte sich diesbezüglich keine Signifikanz in den Analysen der vorliegenden Arbeit.

Es ist auffällig, dass bei der Untersuchung des OS die Kurvenverläufe wenig divergieren und sich sogar mehrfach überkreuzen, so dass keine eindeutige Aussage über einen Überlebensvorteil gemacht werden kann. Nach 36 Monaten Nachbeobachtungszeit standen zudem in keiner der verschiedenen Analysen nichtzensierte lebende Patienten zur Untersuchung zur Verfügung, wodurch auch hier die Aussagekraft stark limitiert ist. Desgleichen umspannen auch die Konfidenzintervalle der verschiedenen Jahres-Raten sehr breite Bereiche, insbesondere nach drei Jahren.

Lediglich beim Vergleich des OS in Abhängigkeit von Rituximab als Bestandteil der Vortherapie separieren die Kurvenverläufe deutlich, jedoch nicht signifikant $(p=0,14)$. Interessanterweise zeigen hier die Patienten ohne vorangegangene Rituximabtherapie, welche also erstmalig im Rahmen der RIT Rituximab erhalten hatten, ein besseres Überleben, obwohl eine Überlegenheit der Patienten mit Rituximab als Bestandteil der Vortherapie zu erwarten gewesen wäre. Es ist jedoch zu beachten, dass es sich um eine sehr kleine Subgruppe $(n=7)$ handelt und auf Grund mangelnder Kontrollmöglichkeiten der Registerdaten eine vorherige Selektion hier nicht auszuschließen ist. Somit ist auch hier die Aussagekraft dieser Untersuchung stark limitiert und die Validität zu unsicher.

Auch in den Subgruppenanalysen zur TTP wird bei nahezu parallelen sowie gering bis gar nicht divergierenden Kurvenverläufen, welche bei der Auftragung weniger dokumentierter Ereignisse in kleinen Patientenkollektiven entstanden, sowie sehr breiter Konfidenzintervalle und fehlenden "patients at risk" nach 36 Monaten deutlich, dass der Informationswert hier ebenfalls gering ist. 
Es lässt sich also feststellen, dass die Untersuchung der verschiedenen Subgruppen des "First line"-Patientenkollektivs keine eindeutige oder signifikante Aussage zu eventuellen Überlebensvorteilen zulässt, obwohl diese beispielsweise anhand der Ergebnisse der RICOVER60- und MInT-Studie zu erwarten gewesen wären (Pfreundschuh et al. 2006; Pfreundschuh et al. 2008b). Begründet wird dies durch bereits $\mathrm{zu}$ Beginn kleine Patientenzahlen und im Verlauf zu wenigen bis gar keinen "patients at risk" sowie eine zu große Abhängigkeit beziehungsweise fehlende Randomisierung. Da somit die Zuverlässigkeit und Validität der erzielten Daten nicht sicher ist, wurde im Folgenden auf einen expliziten Vergleich dieser Auswertungen mit klinischen Studien verzichtet.

Die Ergebnisse einer eingangs beschriebenen klinischen Studie zur Analyse des Sicherheitsprofils von ${ }^{90} \mathrm{Y}$-IT charakterisieren die zu erwartenden unerwünschten Arzneimittelwirkungen bei der Anwendung von RIT bei indolenten sowie aggressiven NHL (Witzig et al. 2003). Dabei zeigte sich, dass hauptsächlich Myelosuppression sowie Hämatotoxizität zu erwarten waren, so dass in der vorliegenden Arbeit die Nadire der verschiedenen Zellreihen des Blutes bestimmt wurden (siehe Kapitel 3.4, S.58). Nicht hämatologische Wirkungen traten tatsächlich seltener auf (6,5\% des Gesamtkollektivs) und waren zumeist, wie auch Witzig et al. beschreiben, milder.

Um den Informationswert der Auswertungen der Nadirbestimmung einschätzen zu können, ist $\mathrm{zu}$ bemerken, dass nicht für alle Registerpatienten entsprechende Blutbildkontrollen dokumentiert wurden (zwischen 59,5-82,8\% im Gesamtkollektiv) und somit teilweise lediglich unsichere Aussagen getroffen werden können. Des Weiteren sind mögliche Fehleingaben, welche beispielsweise durch nicht erfolgte Umrechnung in die im Register geforderten Einheiten entstehen konnten, nicht auszuschließen. Um die Rate versehentlich inkorrekt dokumentierter Daten möglichst gering $\mathrm{zu}$ halten, wurden 26 Patienten beziehungsweise 15 Patienten des Gesamtkollektivs aus dieser Auswertung genommen, da bei ihnen ein Nadir der Neutrophilen Granulozyten beziehungsweise der Leukozyten von 0/ $\mu 1$ beschrieben wurde und dies höchstwahrscheinlich durch eine versehentliche Fehleingabe entstanden ist. Bei einem dennoch sehr breiten Bereich zwischen den minimal und maximal dokumentierten Werten war die Bestimmung des jeweiligen Medians besonders entscheidend, um den Einfluss ausreißender Werte zu minimieren.

Im "First line"-Patientenkollektiv lag der Anteil an Dokumentationen höher als im Gesamtkollektiv (82,0-91,7 \%) und es mussten auch keine Patienten ausgeschlossen werden, so dass das hier analysierte Nebenwirkungsprofil eine größere Glaubwürdigkeit besitzt. Da 
die ermittelten Risikoprofile beider Kollektive jedoch nahezu deckungsgleich sind (siehe Tabelle 3.13, S.60 und Tabelle 3.14, S.62), scheinen auch beide die bei der Anwendung von RIT entstehenden unerwünschten Arzneimittelwirkungen regelgerecht wiederzugeben. Es waren jeweils nach einer medianen Dauer zwischen 33,0-42,5 Tagen eine mediane Neutropenie Grad IV, eine mediane Leukozytopenie Grad III, eine mediane Thrombozytopenie Grad IV sowie eine mediane Anämie Grad I beziehungsweise Grad II aufgetreten und in beiden Gruppen erreichten die Blutwerte nach einer medianen Erholungszeit von 82,0 Tagen wieder Normwerte.

Zusammenfassend lässt sich sagen, dass das im RIT-Register untersuchte Patientenkollektiv bezüglich der Patienten- und Lymphomcharakteristika gut vergleichbar mit den Patienten aus der klinischen Alltagsroutine ist und die beschriebenen Auswertungen verlässlich und glaubwürdig sind. Weiterhin ist auch die Validität und Zuverlässigkeit der Analysen zum Therapieansprechen und der Nebenwirkungen gegeben. Einzig die Auswertungen der Langzeitdaten über zwölf Monate hinaus sowie die Subgruppenanalysen der "First line"Patienten besitzen eine geringe Aussagekraft und sind in ihrer Glaubwürdigkeit eingeschränkt.

\subsection{Klinische Ergebnisse des RIT-Registers im Vergleich mit klinischen Studien zur Anwendung von RIT}

Im Folgenden sollen nun die im RIT-Register dokumentierten und in der vorliegenden Arbeit analysierten Therapieergebnisse mit den Untersuchungsergebnissen aus aktuellen und zentralen klinischen Studien zur Anwendung von RIT bei DLBCL bezüglich der Durchführbarkeit, Sicherheit und Ansprechraten, soweit möglich, verglichen werden. Dabei wird zuerst das Gesamtkollektiv des RIT-Registers mit zwei klinischen Studien verglichen, in welchen ein ähnliches Patientenkollektiv untersucht wurde. Anschließend wird dann das "First line"-Patientenkollektiv mit zwei weiteren Studien verglichen, in welchen ebenfalls die Anwendung von RIT als Erstlinientherapie untersucht wurde. Wie bereits beschrieben, werden dabei die Ergebnisse der Subgruppenanalysen des "First line"-Patientenkollektivs zumeist nicht in den Vergleich miteinbezogen, da diese über eine zu geringe Aussagekraft verfügen. Die Auswertungen der Verlaufsbeobachtungen werden zur Vollständigkeit in den Vergleichen mit aufgeführt, obwohl diese nach mehr als zwölf Monaten Nach- 
beobachtungszeit stark an Validität und Aussagekraft verlieren und somit für einen direkten Vergleich nicht geeignet sind.

Zuletzt werden die im RIT-Register ausgewerteten unerwünschten Arzneimittelwirkungen mit den Ergebnissen verschiedener klinischer Studien verglichen.

\subsubsection{Die Ergebnisse des Gesamtkollektivs im Vergleich mit den Ergebnissen zweier klinischer Studien}

Eine der ersten maßgeblichen Studien zur Untersuchung von RIT bei CD20-positiven B-ZellLymphomen stellt die Veröffentlichung von Gordon et al. dar (Gordon et al. 2004). Unter anderem wurden hierbei zwölf Patienten mit rezidivierten DLBCL und zur Anwendung von RIT geeignetem Krankheitsstatus (siehe Kapitel 1.2.2, S.16) mit ${ }^{90}$ Y-IT behandelt. Die hierbei angewandte Energiedosis lag zwischen 0,2-0,4 mCi/kg Körpergewicht und war somit mit der medianen Dosis von $0,34 \mathrm{mCi} / \mathrm{kg}$ Körpergewicht der Patienten des RIT-Registers vergleichbar. Alle zwölf Studienpatienten waren chemotherapeutisch vorbehandelt, im Median mit zwei Chemotherapieprotokollen. Auch hier zeigt sich demgemäß eine Analogie zu dem Patientenkollektiv des RIT-Registers, welches bei 187 Patienten (87,0\%) zwischen einer und drei vorherigen Chemotherapielinien aufweist. Nach einer längeren medianen Nachuntersuchungszeit von 28,5 Monaten (im Vergleich zu 11,5 Monate beim RIT-Register) hatten 33,0 \% der Studienpatienten eine CR und 17,0\% eine CRu erreicht. Mit einem Anteil von $8,0 \%$ PR lag die ORR in der Studie bei 58,0 \%. Von den im RIT-Register untersuchten Patienten wurde im Vergleich dazu eine höhere CR-Rate von 54,4\% sowie eine bessere ORR von 63,3\% erreicht. Mit einer Spanne von 0,9 bis > 68,6 Monaten lag die TTP der klinischen Studie im Median bei 4,6 Monaten. Diese recht kurze mediane TTP lässt sich, laut Gordon et al., durch einen frühen Progress vieler Patienten erklären, die kein Ansprechen auf die RIT gezeigt haben. Ein direkter Vergleich der medianen TTP ist, auf Grund des nach über dreijähriger Nachuntersuchungszeit noch nicht erreichten 50\%-Quantils der Registerpatienten, nicht möglich, jedoch zeigt der Kurvenverlauf auch hier in den ersten fünf Beobachtungsmonaten den schnellen Progress der RIT-refraktären Patienten und im Weiteren einen stabilen Verlauf mit wenig Progressen der Patienten, die ein gutes Ansprechen auf die RIT zeigen (siehe Abbildung 3.10, S.50).

Des Weiteren sollen die in dieser Arbeit präsentierten Therapieergebnisse mit denen einer zweiten zentralen Studie über die Sicherheit und Durchführbarkeit von ${ }^{90}$ Y-IT bei rezidivierten oder therapierefraktären CD20-positiven DLBCL aus der Arbeitsgruppe um 
Morschhauser verglichen werden (Morschhauser et al. 2007). Die in dieser Studie untersuchten 102 Patienten waren mindestens 60 Jahre alt, für eine autoSZT ungeeignet und erfüllten dafür aber die Kriterien für eine radioimmuntherapeutische Behandlung. Sie waren entweder mit CHOP (oder einem vergleichbaren Therapieprotokoll) oder mit R-CHOP (oder einem vergleichbaren Therapieprotokoll in Kombination mit Rituximab) vortherapiert worden und hatten im Anschluss eine RIT mit einer Energiedosis von 0,4 mCi/kg Körpergewicht erhalten. Nach einer medianen Nachuntersuchungszeit von 21,7 Monaten zeigte sich für die Patienten, die nur CHOP als Vortherapie erhalten hatten $(n=76)$, eine ORR von 53,0\% $(\mathrm{CR}=28,0 \%, \mathrm{CRu}=5,0 \%, \mathrm{PR}=20,0 \%)$. Im Vergleich zu der zuvor beschriebenen Studie lag hier zwar der Anteil der CR um 17,0 \% niedriger, die ORR war jedoch von ähnlicher Größenordnung. Die kleinere Patientengruppe $(n=26)$ der Studie, die Rituximab bereits als Bestandteil der Vortherapie hatte, konnte dagegen nicht so gute Therapieergebnisse aufweisen. Bei ihnen lag die ORR bei lediglich 19,0\% mit insgesamt 12,0\% CR und CRu. Morschauser et al. zufolge könnte dieses Ergebnis mit einem initial schlechteren IPI (IPI $>2=61,0 \%$ ) und einer größeren Tumormasse und damit schlechteren Prognose innerhalb dieser Gruppe begründet werden. Dieser Annahme entsprechend zeigt sich, dass die Patienten des RIT-Registers, die zum Großteil $(\mathrm{n}=185 ; 86,0 \%)$ auch Rituximab als Vortherapie hatten, jedoch ein besseres Risikoprofil aufwiesen (lediglich 39,6\% der Patienten, deren IPI dokumentiert wurde, beziehungsweise 26,5\% des Gesamtkollektivs hatten einen IPI > 2), letztlich auch eine bessere ORR und CR-Rate erreicht hatten. Analog dazu war auch das mediane OS der Studienpatienten, die mit R-CHOP vortherapiert waren, mit 4,6 Monaten um 22,6 Monate und das mediane PFS mit 1,6 Monaten um 21,8 Monate kürzer als beim Gesamtkollektivs des Registers.

Aber auch die Patientengruppe, welche in der Studie nur CHOP erhalten hatte und über die besseren Ansprechraten verfügte, erreichte mit einem medianen OS von 21,4-22,4 Monaten und einem medianen PFS von 3,5-5,9 Monaten (jeweils in Abhängigkeit zum Ansprechen auf die Vortherapie), nicht das mediane OS (27,2 Monate) und das mediane PFS (23,4 Monate) der gesamten Registerpatienten.

Zur besseren Übersicht und besseren Vergleichbarkeit wurde in Tabelle 4.2 die ORR, die Anzahl an CR und CRu, das mediane OS, die mediane TTP und das mediane PFS (sofern vorliegend) der zwei zentralen Studien sowie der vorliegenden Arbeit aufgeführt. Vergleichend wird deutlich, dass das RIT-Register über das größte Patientenkollektiv mit den besten Ansprechraten auf eine mit heterogenen Therapieprotokollen kombinierte RIT verfügt. 
Es ist außerdem offenkundig, dass das längste mediane OS und insbesondere das längste mediane PFS bei den im RIT-Register analysierten Patienten erzielt wurde.

\begin{tabular}{|c|c|c|c|}
\hline & RIT-Register & $\begin{array}{c}\text { Gordon et al. } \\
2004\end{array}$ & $\begin{array}{c}\text { Morschhauser et al. } \\
2007\end{array}$ \\
\hline $\begin{array}{c}\text { Anzahl Patienten } \\
{[\mathrm{n}]}\end{array}$ & 215 & 12 & $\begin{array}{l}76 \\
26\end{array}$ \\
\hline $\begin{array}{c}\text { ORR } \\
{[\%]}\end{array}$ & 63,3 & 58,0 & $\begin{array}{l}53,0 \\
19,0\end{array}$ \\
\hline $\begin{array}{c}\text { CR + CRu } \\
{[\%]}\end{array}$ & 54,4 & 50,0 & $\begin{array}{l}33,0 \\
12,0\end{array}$ \\
\hline $\begin{array}{l}\text { PR } \\
{[\%]}\end{array}$ & 8,9 & 8,0 & $\begin{array}{c}20,0 \\
7,0\end{array}$ \\
\hline $\begin{array}{c}\text { Medianes OS } \\
\text { [Monate] }\end{array}$ & 27,2 & n.d. & $\begin{array}{c}21,4-22,4 \\
4,6\end{array}$ \\
\hline $\begin{array}{c}\text { Mediane TTP } \\
\text { [Monate] }\end{array}$ & $\begin{array}{c}>36,0 \\
\text { (geschätzt) }\end{array}$ & 4,6 & n.d. \\
\hline $\begin{array}{c}\text { Medianes PFS } \\
\text { [Monate] }\end{array}$ & 23,4 & n.d. & $\begin{array}{c}3,5-5,9 \\
1,6\end{array}$ \\
\hline
\end{tabular}

n.d. = nicht dokumentiert

Tabelle 4.2: klinische Ergebnisse des RIT-Registers und zweier klinischer Studien im Vergleich (Gordon et al. 2004; Morschhauser et al. 2007). 


\subsubsection{Die Ergebnisse des "First line"-Patientenkollektivs im Vergleich mit den Ergebnissen zweier klinischer Studien}

Das "First line"-Patientenkollektiv des RIT-Registers, welches in der vorliegenden Arbeit gesondert ausgewertet wurde (siehe Kapitel 3.2.1, S.34 und Kapitel 3.4.1, S.61), ist ausreichend gut vergleichbar mit den Studienpatienten zweier Veröffentlichungen von Zinzani et al.. Diese Studien untersuchten die Durchführbarkeit und Sicherheit von RIT als Konsolidierung nach sechs Zyklen CHOP beziehungsweise vier Zyklen R-CHOP-21 bei DLBCL-Patienten, welche mindesten 60 Jahre alt waren (Zinzani et al. 2008; Zinzani et al. 2010). Die wichtigsten Gemeinsamkeiten dieser Studienpatienten und der "First line"Patienten liegen in der Indikationsstellung als Konsolidierung in Erstlinientherapie, welche für alle Studienpatienten $(n=20$ und $n=55)$ und den Großteil der Registerpatienten $(n=62$; $86,1 \%)$ bestand sowie in den Protokollen der Vortherapien, welche bei 56 Patienten (90,3\%) des RIT-Registers mit Indikation zur Konsolidierung und 55 der Studienpatienten den Antikörper Rituximab als Therapiebestandteil hatten. Die übrigen 20 Studienpatienten hatten lediglich CHOP als Vortherapie erhalten, so wie auch fünf Patienten des Registers mit nur CHOP und ein weiterer Patient mit einem anderen Therapieprotokoll ohne Rituximab vorbehandelt waren. Des Weiteren wurde auch die RIT mit einer ähnlichen Energiedosis in den verschiedenen Kollektiven verabreicht. Während Zinzani et al. in Abhängigkeit der Thrombozytenanzahl 0,3 oder 0,4 mCi/kg Körpergewicht anwendeten, lag die mediane Dosis der "First line"-Patienten bei 0,35 mCi/kg Körpergewicht.

Die größten Unterschiede zwischen den beiden Studien und der Registerauswertung liegen dagegen in den jeweiligen Patientencharakteristika. Die Studienpatienten von Zinzani et al. waren alle mindestens 60 Jahre alt (Median $=68$ und 70 Jahre) und hatten größtenteils einen IPI $\geq 2(75,0 \%$ der Patienten mit CHOP und $100,0 \%$ der Patienten mit R-CHOP in der Vortherapie). Bei den "First line"-Patienten zeigte sich demgegenüber zwar auch ein medianes Erkrankungsalter von immerhin 65 Jahren, es waren jedoch auch 22 Patienten (30,6\%) jünger als 60 Jahre. Des Weiteren hatten nur 47 Patienten $(65,3 \%)$ zwei oder mehr Risikofaktoren (IPI $\geq 2$ ) aufzuweisen. Es bestand also ein gering besseres Risikoprofil der "First line"-Patienten gegenüber den Studienpatienten, dennoch sollten die Ähnlichkeiten der Patientenkollektive und Therapieansätze ausreichend für einen Vergleich der beiden Studien mit den klinischen Ergebnissen der "First line"-Patientengruppe sein.

Besonders in der Studie von 2008, in der sechs Zyklen CHOP vor einer Konsolidierung mit RIT bei 20 DLBCL-Patienten eingesetzt wurden (Zinzani et al. 2008), konnten achtbare 
Therapieergebnisse erzielt werden. So wurden hier eine ORR von 100,0 \% und eine CR-Rate von 95,0 \% erreicht. Die 2-Jahres-OS-Rate wurde von Zinzani et al. bei einer medianen Nachuntersuchungszeit von 15,0 Monaten auf 95,0 \% und die 2-Jahres-PFS-Rate auf 75,0 \% geschätzt.

Aber auch die Anwendung von vier Zyklen R-CHOP-21 vor einer konsolidierenden RIT bei 55 älteren Patienten mit einem IPI $\geq 2$ bewirkte in der zweiten Studie eine ORR von 80,0\% (Zinzani et al. 2010). Hier zeigte sich bei 73,0 \% der Patienten eine CR, während nur 58,0 \% der Patienten eine CR nach der Vortherapie mit R-CHOP erreicht hatten. Die ORR hatte jedoch nach R-CHOP bereits bei 89,0\% gelegen und hatte sich somit nach der RIT um 9,0\% verschlechtert. Nach einer medianen Nachuntersuchungszeit von 18,0 Monaten schätzten Zinzani et al. die 2-Jahres-OS-Rate auf 86,0 \% und die 2-Jahres-PFS-Rate auf 85,0 \%.

Im Vergleich zu diesen beiden Studien erreichten die "First line"-Patienten des RIT-Registers mit einer ORR von 83,3\% und 76,4\% CR zwar ein leicht besseres Ansprechen auf die RIT als die Patienten der Studie aus 2010, jedoch nicht die ungewöhnlich guten Ergebnisse der ersten Studie. Desgleichen liegt auch das Therapieansprechen der mindestens 60-jährigen Registerpatienten mit einer ORR von 84,0 \% in einem ähnlichen Bereich. Weiterhin erstreckt sich auch das OS und PFS nach zwei Jahren der Registerpatienten (76,0 \%, 69,0 \%) unterhalb des jeweils geschätzten 2-Jahres-OS und -PFS beider Studien, wobei, wie bereits beschrieben wurde, die limitierte Aussagekraft dieser Auswertung der Registeranalyse bedacht werden muss.

In Tabelle 4.3 sind die Therapieergebnisse der beiden klinischen Studien und die des RITRegisters für "First line"-Patienten zusammenfassend und vergleichend dargestellt. 


\begin{tabular}{|c|c|c|c|}
\hline & "First line" & $\begin{array}{c}\text { Zinzani et al. } \\
2008\end{array}$ & $\begin{array}{c}\text { Zinzani et al. } \\
2010\end{array}$ \\
\hline $\begin{array}{c}\text { Anzahl Patienten } \\
{[\mathrm{n}]}\end{array}$ & 72 & 20 & 55 \\
\hline $\begin{array}{l}\text { ORR } \\
{[\%]}\end{array}$ & 83,3 & 100,0 & 80,0 \\
\hline $\begin{array}{l}\text { CR } \\
{[\%]}\end{array}$ & 76,4 & 95,0 & 73,0 \\
\hline $\begin{array}{l}\text { PR } \\
{[\%]}\end{array}$ & 6,9 & 5,0 & 7,0 \\
\hline $\begin{array}{c}\text { 2-Jahres-OS } \\
\text { (KI) } \\
{[\%]}\end{array}$ & $\begin{array}{c}76,0 \\
(58,9-86,3)\end{array}$ & 95,0 & 86,0 \\
\hline $\begin{array}{c}\text { 2-Jahres-PFS } \\
\text { (KI) } \\
{[\%]}\end{array}$ & $\begin{array}{c}69,0 \\
(53,9-80,5)\end{array}$ & 75,0 & 85,0 \\
\hline
\end{tabular}

Tabelle 4.3: Therapieansprechen der "First line"-Patienten des RIT-Registers auf die RIT im Vergleich mit zwei klinischen Studien (Zinzani et al. 2008; Zinzani et al. 2010).

\subsubsection{Unerwünschte Arzneimittelwirkungen der RIT bei Registerpatienten im Vergleich mit klinischen Studien}

Um die Sicherheit der RIT im klinischen Alltag zu zeigen, sollen hier nun die unerwünschten Arzneimittelwirkungen der Registerpatienten mit solchen bereits beschriebener, beispielhafter Studienkollektive verglichen werden.

Es zeigt sich dabei, dass die jeweils häufigste unerwünschte Wirkung der RIT, nämlich eine durch Suppression des Knochenmarks und Hämatotoxizität ausgelöste Erniedrigung der Blutbestandteile, sowohl bei den Registerpatienten als auch bei den Patienten beider oben beschriebenen Studien von Zinzani et al. nach einer ähnlich langen medianen Dauer nach Applikation der RIT aufgetreten waren. So lag die mediane Zeit bis zur vollständigen Ausbildung der Nadire der verschiedenen Zellreihen im Gesamtkollektiv jeweils zwischen 
33,0 und 40,0 Tagen, im "First line"-Kollektiv zwischen 33,0 und 42,5 Tagen und bei Zinzani et al. zwischen 35,0 und 43,0 (Zinzani et al. 2008) beziehungsweise 32,0 und 41,0 Tagen (Zinzani et al. 2010). In der Analyse des Sicherheitsprofils bei der Anwendung von RIT bei indolenten sowie aggressiven NHL von Witzig et al., waren die jeweiligen Nadire dagegen erst nach sieben bis neun Wochen (45,0-63,0 Tagen) aufgetreten (Witzig et al. 2003).

Weiterhin zeigt sich, dass besonders die entwickelte Thrombozytopenie und Neutropenie der Registerpatienten (jeweils Grad IV) stärker als die der Studienpatienten (jeweils Grad III) ausgeprägt war und die Erniedrigung der Zellreihen in der RIT-Register-Analyse längere Zeit zur Regeneration benötigte. Die mediane Dauer bis zur Überwindung von dritt- oder viertgradigen Zytopenien lag bei den Studienpatienten von Zinzani et al. zwischen 21 und 32 Tagen, wobei jedoch keine Angaben über die Dauer bis zum Erreichen normwertiger Blutwerte gegeben sind. Bei Witzig et al. hatten sich die Zellreihen bereits nach einer bis vier Wochen (7,0-28,0 Tagen) wieder regeneriert, während im RIT-Register im Median 82,0 Tage in einem Wertebereich von 42 bis 599 Tagen für eine vollständige Erholung der Blutwerte vonnöten waren.

In den Tabellen 4.4a-c sind die detaillierten Nebenwirkungsprofile der Studienpatienten von Zinzani et al. sowie der "First line"-Patienten vergleichend aufgelistet, da diese Patientenkollektive über die meisten Gemeinsamkeiten und somit über die größte Vergleichbarkeit verfügen.

\begin{tabular}{|c|c|c|c|}
\hline Neutrophile Granulozyten & "First line" & $\begin{array}{c}\text { Zinzani et al. } \\
\mathbf{2 0 0 8}\end{array}$ & $\begin{array}{c}\text { Zinzani et al. } \\
\mathbf{2 0 1 0}\end{array}$ \\
\hline $\begin{array}{c}\text { Mediane Zeit } \\
\text { bis zum Auftreten des Nadirs } \\
\text { (Verteilung) } \\
{[\text { Tage] }}\end{array}$ & 37 & 39 & 37 \\
\hline $\begin{array}{c}\text { Medianer Nadir } \\
(\text { Verteilung) } \\
{[/ \mu \mathrm{l}]}\end{array}$ & $150-$ Grad IV & $800-\mathrm{Grad}$ III & $700-\mathrm{Grad}$ III \\
& $(0,2-2.800)$ & $(25-60)$ & $(210-2.950)$ \\
\hline
\end{tabular}

Tabelle 4.4a: Maximal verminderte Anzahl der Neutrophilen Granulozyten der "First line"Patienten im Vergleich mit zwei klinischen Studien (Zinzani et al. 2008; Zinzani et al. 2010). 


\begin{tabular}{|c|c|c|c|}
\hline Thrombozyten & "First line" & $\begin{array}{c}\text { Zinzani et al. } \\
2008\end{array}$ & $\begin{array}{c}\text { Zinzani et al. } \\
2010\end{array}$ \\
\hline $\begin{array}{l}\text { Mediane Zeit } \\
\text { bis zum Auftreten des Nadirs } \\
\text { (Verteilung) } \\
\text { [Tage] }\end{array}$ & $\begin{array}{c}33 \\
(15-63)\end{array}$ & $\begin{array}{c}35 \\
(20-43)\end{array}$ & $\begin{array}{c}32 \\
(19-38)\end{array}$ \\
\hline $\begin{array}{c}\text { Medianer Nadir } \\
\text { (Verteilung) } \\
{[/ \mu \mathrm{l}]}\end{array}$ & $\begin{array}{c}3.071 \text { - Grad IV } \\
(5-140.000)\end{array}$ & $\begin{array}{l}42.000 \text { - Grad III } \\
(9.000-187.000)\end{array}$ & $\begin{array}{l}37.000 \text { - Grad III } \\
(10.000-121.000)\end{array}$ \\
\hline
\end{tabular}

Tabelle 4.3b: Maximal verminderte Anzahl der Thrombozyten der "First line"-Patienten im Vergleich mit zwei klinischen Studien (Zinzani et al. 2008; Zinzani et al. 2010).

\begin{tabular}{|c|c|c|c|}
\hline Hämoglobin & "First line" & $\begin{array}{c}\text { Zinzani et al. } \\
\mathbf{2 0 0 8}\end{array}$ & $\begin{array}{c}\text { Zinzani et al. } \\
\mathbf{2 0 1 0}\end{array}$ \\
\hline $\begin{array}{c}\text { Mediane Zeit } \\
\text { bis zum Auftreten des Nadirs } \\
\text { (Verteilung) } \\
\text { [Tage] }\end{array}$ & 43 & 43 & 41 \\
\hline $\begin{array}{c}\text { Medianer Nadir } \\
\text { (Verteilung) } \\
\text { [g/dl] }\end{array}$ & $10,0-$ Grad I & $11,8-$ Grad I & $11,3-$ - Grad I \\
\hline
\end{tabular}

Tabelle 4.3c: Maximal verminderter Gehalt an Hämoglobin der "First line"-Patienten im Vergleich mit zwei klinischen Studien (Zinzani et al. 2008; Zinzani et al. 2010).

Weiterhin wurden sowohl in den verschiedenen bereits genannten klinischen Studien als auch bei der Auswertung des RIT-Registers nur wenige (6,5\% des Gesamtkollektivs) und meist milde, nichthämatologische unerwünschte Arzneimittelwirkungen beobachtet. Die dabei am häufigsten betroffenen Organsysteme waren der Gastrointestinaltrakt, der Respirationstrakt, die Haut sowie das Nervensystem (Morschhauser et al. 2007; Zinzani et al. 2008). Die Inzidenz an sekundären, malignen Erkrankungen, insbesondere dem MDS oder der akuten myeloischen Leukämie (AML), war bei den in dieser Arbeit untersuchten Patienten 
gegenüber denen anderer Untersuchungen nicht erhöht. Ein Registerpatient hatte in Folge der RIT ein MDS entwickelt und bei einem weiteren wurde der Verdacht auf ein MDS gestellt $(\mathrm{n}=2 ; 0,9 \%)$. Bei Gordon et al. waren zwei Patienten $(4,0 \%)$ an einem MDS erkrankt (Gordon et al. 2004), während in der Studie von Witzig et al. fünf Patienten (1,0\%) nach der radioimmuntherapeutischen Behandlung ein MDS oder eine AML entwickelten (Witzig et al. 2003). In einer weiteren Studie zur Untersuchung der Inzidenz von MDS oder AML bei 746 NHL-Patienten, welche mit ${ }^{90}$ Y-IT behandelt wurden, zeigten sich bei 19 Patienten $(2,5 \%)$ eine Neuerkrankung an einem MDS oder einer AML. Dies ist laut Czuczman et al. vergleichbar mit der Inzidenz nach einer extensiven Polychemotherapie bei NHL und somit nicht erhöht nach der RIT (Czuczman et al. 2007).

\subsubsection{Bedeutung dieser Ergebnisse im wissenschaftlichen Kontext}

Zusammenfassend zeigen die Vergleiche der Therapieergebnisse des RIT-Registers mit den Ergebnissen zentraler klinischer Studien zur Anwendung der RIT bei DLBCL, dass die erzielten Resultate der Registerpatienten mindestens gleichermaßen erfolgversprechend wie die der Studien sind. Bei einer ausreichend großen Vergleichbarkeit der Patientenkollektive erreichten die Patienten des RIT-Registers zumeist sogar eine bessere ORR und CR-Rate. Einzig die kleine Studie von Zinzani et al. erzielte Therapieergebnisse, die denen des RITRegisters überlegen waren. Hierbei ist jedoch zu bemerken, dass die ORR der besagten Studie bereits nach der Vortherapie mit CHOP bei 100,0 \% und somit fragwürdig hoch lag. In der vorliegenden Arbeit wurde das Ansprechen auf die erhaltene Vortherapie der Konsolidierung nicht dokumentiert und analysiert, jedoch ist im Allgemeinen nicht zu erwarten, dass in einem größeren Patientenkollektiv die chemotherapeutische Anbehandlung alleine eine solch hohe Ansprechrate bewirkt. So führte beispielsweise die Anwendung von sechs Zyklen CHOP-14 in der RICOVER60-Studie, auf die im folgenden Kapitel näher eingegangen wird, lediglich zu einer ORR von 75,0 \% (Pfreundschuh et al. 2006).

Ferner zeigt sich, dass die Patienten im RIT-Register zwar die größte Patientengruppe bilden, jedoch über die kürzeste mediane Nachbeobachtungszeit verfügen (11,5 Monate im Gesamtkollektiv und 12,1 Monate bei den "First line"-Patienten im Vergleich zu 15,0-28,5 Monaten bei den Studienpatienten). Dadurch, sowie aus den weiter oben bereits diskutierten Gründen, ist der Informationswert der Langzeitergebnisse über zwölf Monate hinaus im RITRegister begrenzt und ein Vergleich mit den Langzeitergebnissen aus den diskutierten Studien nur bedingt aussagekräftig. So sind einerseits die medianen Überlebenszeiten der gesamten 
Registerpatienten denen der Studienpatienten von Gordon et al. und Morschhauser et al. überlegen, während andererseits die prozentualen Überlebensraten nach zwei Jahren der "First line"-Patienten denen der Studienpatienten unterlegen sind, obwohl das Therapieansprechen jeweils in den Registeranalysen besser als das der Studien (außer Zinzani et al. 2010) war. Da in den genannten Studien entweder die medianen oder die prozentualen Langzeitdaten beschrieben wurden, kann kein übergreifender Vergleich der Langzeitverläufe zwischen Gesamtkollektiv, "First line"-Patienten und Studienpatienten durchgeführt werden. Somit kann nicht eindeutig geklärt werden, ob diese Ergebnisse tatsächlich für einen schlechteren Langzeitverlauf der RIT bei "First line"-Patienten sprechen oder ob sie durch die wenigen "patients at risk", die zur Berechnung des 2-Jahres-OS und -PFS der Registerpatienten zur Verfügung standen, bedingt sind.

Bezüglich der Sicherheit der Anwendung von RIT lässt sich ferner feststellen, dass lediglich geringe Abweichungen zwischen dem alltäglichen klinischen Gebrauch, welcher im RITRegister erfasst wurde, und den kontrollierten Studien bestanden. Die durch die myelosuppressive Wirkung der RIT in Kombination mit einer Polychemotherapie entstandenen Zytopenien der Registerpatienten waren zwar stärker ausgeprägt und, soweit anhand der vorliegenden Daten abschätzbar, von längerer Dauer als die der Studienpatienten, jedoch letztendlich ebenfalls reversibel. Die Inzidenz sekundärer maligner Erkrankungen war hier wie auch in den Studien nicht erhöht. 


\subsection{Klinische Ergebnisse der RIT bei Registerpatienten im Vergleich zu den aktuellen Leitlinien der Therapie von DLBCL}

Da momentan noch keine Zulassung der RIT bei DLBCL besteht, empfehlen die aktuellen Therapieleitlinien für die drei Patientengruppen jüngere Patienten mit niedrigem Risiko $(<60$ Jahre, IPI $\leq 2)$, jüngere Hochrisikopatienten ( $<60$ Jahre, IPI $>2)$ und ältere Patienten $(\geq 60$ Jahre) jeweils eine Polychemotherapie nach dem CHOP-Protokoll kombiniert mit dem CD20-Antikörper Rituximab. Dabei erzielen sechs Zyklen CHOP mit acht Zyklen Rituximab (6 x CHOP-14 $+8 \times \mathrm{R})$ bei der älteren Patientengruppe und sechs Zyklen R-CHOP im dreiwöchigen Rhythmus (R-CHOP-21) bei jüngeren Niedrigrisikopatienten die besten Therapieergebnisse und sind somit als Therapiestandard anzusehen (Held et al. 2011). Für jüngere Patienten mit hohem Risiko besteht dagegen momentan noch kein Therapiestandard, da noch kein ausreichend effizienter Therapieerfolg in verschiedenen Studien erzielt wurde. Aus diesem Grund sollten diese Patienten weiterhin bevorzugt in klinische Studien einbezogen werden (Tilly und Dreyling 2010). Die ausschlaggebenden Ergebnisse zur Etablierung dieser Therapieempfehlungen wurden für ältere Patienten durch die GELA (Coiffier et al. 2002; Coiffier et al. 2010) und die RICOVER60-Studie der DSHNHL (Pfreundschuh et al. 2008b) sowie für jüngere Patienten mit niedrigem Risiko im Besonderen durch die MInT-Studiengruppe (Pfreundschuh et al. 2006) erzielt. Im Folgenden soll, soweit möglich, ein Vergleich der zur Aufstellung der Therapieleitlinien maßgeblichen Studien mit den Ergebnissen des RIT-Registers erfolgen, um die Bedeutung der RIT als neue Therapieoption bei der Behandlung von DLBCL zu eruieren. 


\subsubsection{Die Ergebnisse der RICOVER60-Studie und einer Studie der GELA im Vergleich mit den Ergebnissen der RIT-Registeranalyse}

Wie bereits einleitend beschrieben, wurden in der RICOVER60-Studie die besten Therapieergebnisse mit sechs Zyklen R-CHOP-14 in Kombination mit acht Rituximabgaben erzielt, wodurch dieses Schema Einzug in die Therapieleitlinien erhielt (siehe Kapitel 1.1.3.1, S.11). Mit diesem Protokoll waren 306 zuvor unbehandelte Patienten der Studie therapiert worden, 237 von ihnen waren an DLBCL erkrankt. Es wurde also ein Patientenkollektiv von ähnlicher Größe wie das des RIT-Registers $(n=215)$ untersucht, obwohl das mediane Alter von 69 Jahren um sieben Jahre gegenüber dem der Registerpatienten erhöht war. In der Studie waren nur Patienten untersucht worden, die älter als 60 Jahre alt waren, während 95 (44,2 \%) der in der vorliegenden Arbeit untersuchten Patienten jünger als 60 Jahre waren. Die Tumorausbreitung nach Ann-Arbor beziehungsweise die Risikoeinschätzung mittels IPI war in beiden Patientenkollektiven heterogen repräsentiert.

Die mediane Nachuntersuchungszeit der RICOVER60-Studie lag bei annähernd drei Jahren (34,5 Monate) und war somit deutlich länger, als die des RIT-Registers (11,5 Monate). Dabei zeigte sich eine ORR von $82,0 \%(\mathrm{CR}=78,0 \%, \mathrm{PR}=4,0 \%)$ und lediglich für 7,0\% der Patienten ein PD. Damit wurde ein besseres Therapieansprechen als beim Gesamtkollektiv des RIT-Registers erzielt, bei welchem die ORR bei 63,3\% und die PD-Rate bei 23,7 \% lag. Die mindestens 60-jährigen "First line"-Patienten des RIT-Registers dagegen, welche ja, wie auch die Studienpatienten, zuvor unbehandelt waren, erreichten vergleichbare Ansprechraten auf die zumeist konsolidierende RIT (ORR $=84,0 \%, \quad \mathrm{CR}=76,0 \%, \quad \mathrm{PR}=8,0 \%$, $\mathrm{PD}=14,0 \%$ ), wie die Studienpatienten auf die Polychemotherapie mit Rituximab. Betrachtet man jedoch das 3-Jahres-OS und -PFS, zeigt sich, dass sich dieses Ergebnis im Verlauf der Langzeituntersuchung nicht halten kann. Nach drei Jahren lag die OS-Rate der Studienpatienten bei 78,1 \%, während das jeweilige 3-Jahres-OS in der Registeranalyse bei 43,0 \% (Gesamtkollektiv) beziehungsweise 35,0\% ("First line") und 45,0\% ("First line", $\geq$ 60 Jahre) liegt. Wie bereits beschrieben, ist jedoch die Aussagekraft über das 3-Jahres-OS der Registerpatienten stark limitiert und somit ein Vergleich hier nicht unbedingt sinnvoll. Das 1- oder 2-Jahres-OS, welches in der Register-Auswertung einen größeren Informationswert hat, wurde in der RICOVER60-Studie nicht dokumentiert, so dass ein direkter Vergleich nicht möglich ist.

Eine weitere Empfehlung von bis zu acht Zyklen R-CHOP-21 zur Behandlung älterer Patienten mit DLBCL lässt sich auf eine Studie der GELA zurückführen, in der dieses 
Therapieprotokoll einer alleinigen Polychemotherapie ohne Rituximab überlegen war (Coiffier et al. 2002). Wie schon bei der RICOVER60-Studie sind nur mindestens 60-jährige Patienten mit unbehandelten DLBCL in dieser Studie untersucht worden. Desgleichen war in der Studie die Kollektivgröße von 202 Patienten, die R-CHOP-21 erhalten hatten, jedoch nicht das mediane Patientenalter (69 Jahre) mit den Registerdaten analog.

Nach einer doppelt so langen medianen Nachuntersuchungszeit (24,0 Monate) wie die mediane Nachuntersuchungszeit des RIT-Registers (11,5 Monate), zeigten sich ähnliche Therapieerfolge wie auch schon bei der RICOVER60-Studie. Erneut erzielten die Studienpatienten ein besseres Therapieansprechen als die größtenteils mehrfach vorbehandelten Registerpatienten (Gesamtkollektiv), jedoch nicht als die zuvor unbehandelten, älteren "First line"-Patienten. Diese Ergebnisse, wie auch das Therapieansprechen der RICOVER60-Studie, sind in Tabelle 4.5 vergleichend zusammengefasst. Dort findet sich zusätzlich, soweit vorliegend, das jeweilige OS und PFS der untersuchten Patienten nach zwei Jahren, wobei sich zeigt, dass das 2-Jahres-OS der GELA-Studie (70,0 \%) zwar größer als das des Gesamtkollektivs (56,0\%), jedoch kleiner als das der älteren "First line"-Patienten $(84,0 \%)$ ist. Ebenso liegt das 2-Jahres-PFS der Studie (57,0\%) zwischen dem jeweiligen 2-Jahres-PFS der beiden Register-Kollektive (49,0 \% und 69,0 \%). Das 3-Jahres-OS und -PFS des Registers sowie der RICOVER60-Studie wurde in diesem tabellarischen Vergleich nicht mit aufgeführt, da hier der Informationswert der Registeranalyse zu gering und nicht valide genug ist. 
Diskussion

\begin{tabular}{|c|c|c|c|c|}
\hline & $\begin{array}{c}\text { RIT-Register } \\
\text { Gesamtkollektiv }\end{array}$ & $\begin{array}{l}\text { RIT-Register } \\
\text { "First line" } \\
\geq 60 \text { Jahre }\end{array}$ & $\begin{array}{c}\text { RICOVER60- } \\
\text { Studie }\end{array}$ & $\begin{array}{l}\text { Coiffier et al. } \\
\qquad 2002\end{array}$ \\
\hline $\begin{array}{c}\text { Anzahl } \\
\text { Patienten } \\
{[\mathrm{n}]}\end{array}$ & 215 & 50 & $\begin{array}{c}306 \\
(6 \times \mathrm{CHOP}-14+8 \times \mathrm{R})\end{array}$ & $\begin{array}{c}202 \\
(8 x R-C H O P-21)\end{array}$ \\
\hline $\begin{array}{l}\text { ORR } \\
{[\%]}\end{array}$ & 63,3 & 84,0 & 82,0 & 82,0 \\
\hline $\begin{array}{c}\mathrm{CR}+\mathrm{CRu} \\
{[\%]}\end{array}$ & 54,4 & 76,0 & 78,0 & 75,0 \\
\hline $\begin{array}{l}\text { PR } \\
{[\%]}\end{array}$ & 8,9 & 8,0 & 4,0 & 7,0 \\
\hline $\begin{array}{l}\text { PD } \\
{[\%]}\end{array}$ & 23,7 & 14,0 & 7,0 & 9,0 \\
\hline $\begin{array}{c}\text { 2-Jahres-OS } \\
\text { (KI) } \\
{[\%]}\end{array}$ & $\begin{array}{c}56,0 \\
(45,5-64,5)\end{array}$ & $\begin{array}{c}84,0 \\
(69,6-92,2)\end{array}$ & n.d. & 70,0 \\
\hline $\begin{array}{c}\text { 2-Jahres-PFS } \\
\text { (KI) } \\
{[\%]}\end{array}$ & $\begin{array}{c}49,0 \\
(40,1-57,9)\end{array}$ & $\begin{array}{c}69,0 \\
(53,9-80,5) \\
\text { Alle "First line"- } \\
\text { Patienten }\end{array}$ & n.d. & 57,0 \\
\hline
\end{tabular}

n.d. $=$ nicht dokumentiert

Tabelle 4.5: klinische Ergebnisse des RIT-Registers und zweier zentraler Studien, die zu den aktuellen Therapieleitlinien für ältere Patienten mit DLBCL geführt haben, im Vergleich (Coiffier et al. 2002; Pfreundschuh et al. 2008b). 


\subsubsection{Die Ergebnisse der MInT-Studie im Vergleich mit den Ergebnissen der RIT-Registeranalyse}

Einleitend wurde bereits erwähnt, dass durch die Veröffentlichung der MInT-Studie die Überlegenheit von R-CHOP gegenüber CHOP alleine in der Behandlung jüngerer Niedrigrisikopatienten gezeigt werden konnte (Pfreundschuh et al. 2006). Untersucht wurden hierbei 824 Patienten mit unbehandelten DLBCL, von denen 413 Patienten das später in die Therapieleitlinien aufgenommene Chemotherapieprotokoll R-CHOP-21 in sechs Therapiezyklen erhalten haben. Weitere 410 Patienten erhielten sechs Zyklen CHOP-21 ohne Rituximab. Die Patienten waren zwischen 18 und 60 Jahren alt (Median= 47 Jahre) und hatten eine gute Prognose (IPI < 2). Des Weiteren hatte ein Großteil der Studienpatienten zusätzlich eine konsolidierende Strahlentherapie erhalten. Es zeigte sich ein signifikant besserer Therapieerfolg im ersten Studienarm (6 x R-CHOP-21) gegenüber dem Patientenkollektiv des zweiten Studienarms (6x CHOP-21), so dass nach 15 Monaten der Nachuntersuchungszeit die Studie abgebrochen werden musste. Die Patienten mit dem um Rituximab erweiterten Therapieschema wiesen nach drei Jahren ein OS von 93,0\% und ein PFS von $85,0 \%$ auf. Dabei war nach der fast dreijährigen medianen Nachuntersuchungszeit (34,0 Monate) bei 86,0 \% der Studienpatienten eine CR oder CRu beschrieben. Lediglich bei 4,0\% war ein erneuter PD der DLBCL aufgetreten. Ein tabellarischer Vergleich (Tabelle 4.6) dieser Therapieergebnisse mit der Analyse der vorliegenden Arbeit zeigt, dass die besten in der MInT-Studie erreichten Therapieantworten denen des Gesamtkollektivs und auch der "First line"-Patienten, welche jünger als 60 Jahre waren, überlegen sind. Besonders groß stellt sich diese Diskrepanz im 3-Jahres-OS und -PFS dar, wobei diese allerdings für die Registerpatienten nur gering verlässlich sind. 
Diskussion

\begin{tabular}{|c|c|c|c|}
\hline & $\begin{array}{c}\text { RIT-Register } \\
\text { Gesamtkollektiv }\end{array}$ & $\begin{array}{l}\text { RIT-Register } \\
\text { "First line" } \\
\text { < } 60 \text { Jahre }\end{array}$ & MInT-Studie \\
\hline $\begin{array}{c}\text { Anzahl Patienten } \\
{[\mathrm{n}]}\end{array}$ & 215 & 22 & $\begin{array}{c}413 \\
\text { (6xR-CHOP-21) }\end{array}$ \\
\hline $\begin{array}{c}\text { ORR } \\
{[\%]}\end{array}$ & 63,3 & 81,8 & n.d. \\
\hline $\begin{array}{c}\mathrm{CR}+\mathrm{CRu} \\
{[\%]}\end{array}$ & 54,4 & 77,3 & 86,0 \\
\hline $\begin{array}{l}\text { PR } \\
{[\%]}\end{array}$ & 8,9 & 4,5 & n.d. \\
\hline $\begin{array}{l}\text { PD } \\
{[\%]}\end{array}$ & 23,7 & 9,1 & 4,0 \\
\hline $\begin{array}{c}\text { 3-Jahres-OS } \\
\text { (KI) } \\
{[\%]}\end{array}$ & $\begin{array}{c}43,0 \\
(27,9-57,3)\end{array}$ & $\begin{array}{c}29,0 \\
(1,7-69,0)\end{array}$ & 93,0 \\
\hline $\begin{array}{c}\text { 3-Jahres-PFS } \\
\text { (KI) } \\
{[\%]}\end{array}$ & $\begin{array}{c}34,0 \\
(20,6-48,3)\end{array}$ & $\begin{array}{c}32,0 \\
(6,7-61,3) \\
\text { Alle } \\
\text { "First line"-Patienten }\end{array}$ & 85,0 \\
\hline
\end{tabular}

n.d. $=$ nicht dokumentiert

Tabelle 4.6: klinische Ergebnisse des RIT-Registers und der zentralen Studie, die zu den aktuellen Therapieleitlinien für jüngere Patienten mit DLBCL und guter Prognose geführt haben, im Vergleich (Pfreundschuh et al. 2006). 
Wie bereits erwähnt, besteht derzeit kein Therapiestandard zur Behandlung von jüngeren DLBCL-Patienten mit einem intermediär hohen oder hohen Risiko. Es wird empfohlen, diese jungen Hochrisikopatienten bevorzugt in klinische Studien einzubinden, um das bestmögliche Therapieregime noch zu finden (Tilly und Dreyling 2010). Auf Grund der fehlenden allgemeingültigen Therapieempfehlung für jene Patientengruppe wird an dieser Stelle kein Vergleich mit den Ergebnissen des RIT-Registers durchgeführt.

\subsubsection{Bedeutung dieser Ergebnisse im wissenschaftlichen Kontext}

Die Gegenüberstellung von der Registerauswertung und den Ergebnissen der zwei verschiedenen Studien für über 60-jährige Patienten hat ergeben, dass das Gesamtkollektiv des RIT-Registers ein prozentual schlechteres Therapieansprechen auf heterogene Therapieansätze in Kombination mit einer RIT aufweist, als die in den Studien untersuchten Patienten auf eine Polychemotherapie in Kombination mit Rituximab. Dieser Vergleich ist jedoch nur bedingt aussagekräftig, da in diesen Studien lediglich zuvor unbehandelte DLBCL-Patienten untersucht wurden, während im RIT-Registers einzig die "First line"-Patienten eine Erstlinientherapie mit RIT erhalten haben. Das Gesamtkollektiv war dagegen zumeist mehrfach und heterogen vorbehandelt und wies zudem oft rezidivierte oder therapierefraktäre Lymphome und somit einen erwartungsgemäß schlechteren Verlauf auf. Da laut Tilly und Dreyling bei einer konventionellen Polychemotherapie mit Rituximab letztendlich bis zu 30,0 \% der Patienten einen Rückfall erleiden (Tilly und Dreyling 2010), spricht eine ORR von $63,3 \%$ in einem gemischten, mehrfach konventionell vorbehandelten und teilweise rezidivierten beziehungsweise bislang therapierefraktären Patientenkollektiv für eine neue erfolgreiche Therapieoption in diesem Gebiet. Die RIT bietet somit, wie in der vorliegenden Arbeit gezeigt werden konnte, eine neue Möglichkeit für die Behandlung von älteren DLBCL-Patienten, bei denen eine leitliniengerechte Therapie nicht zu einer Remission des Lymphoms oder im weiteren Verlauf zu einem Rezidiv führt.

Betrachtet man den Vergleich der älteren "First line"-Patienten mit den älteren Studienpatienten, so präsentiert sich kein signifikanter Unterschied des jeweiligen Therapieansprechens, sondern lediglich ein geringer Vorteil der Registerpatienten. Die zumeist konsolidierende RIT nach vorheriger Polychemotherapie zeigt sich hier also einer alleinigen Polychemotherapie in Kombination mit Rituximab in der Anwendung als Erstlinientherapie nicht deutlich überlegen. Allerdings weisen die "First line"-Patienten im Langzeitverlauf ein besseres OS und PFS nach zwei Jahren auf als die Patienten der GELA-Studie. Es bleibt 
hierbei jedoch unsicher, ob dies auf einen tatsächlich besseren Langzeitverlauf der Registerpatienten zurückzuführen ist, da die Registerauswertung nach mehr als zwölf Monaten Nachbeobachtungszeit an Aussagekraft und Validität verliert und das Konfidenzintervall dieser Untersuchungen bereits sehr breit ist (siehe Tabelle 3.8, S.45 und Tabelle 3.12, S.58), sodass die bestimmten Werte nicht sicher sind.

In der Untersuchung jüngerer Niedrigrisikopatienten erzielt die MInT-Studie noch bessere Ergebnisse als die Patienten des Gesamt- und sogar des "First line"-Kollektivs (< 60 Jahre), wobei jedoch $\mathrm{zu}$ beachten ist, dass das Studienpatientenkollektiv hier ein besseres Risikoprofil als die Registerpatienten innehatte, da alle Patienten der Studie $(n=413)$ weniger als zwei Risikofaktoren (IPI < 2) aufwiesen. Im Gesamtkollektiv wiesen demgegenüber nur 35 Patienten (16,3\% des Gesamtkollektivs beziehungsweise 24,3\% der Patienten, bei denen eine IPI-Bestimmung möglich war) und bei den "First line"-Patienten nur 14 (19,4\%) ein niedriges Risiko (IPI < 2) auf. Zudem hatten die Studienpatienten größtenteils zusätzlich eine konsolidierende Strahlentherapie erhalten. Dadurch ließe sich folglich das bessere Ergebnis der MInT-Studie gegenüber dem der Registerauswertung erklären, obwohl bei älteren Patienten mit heterogenem Risikoprofil mindestens ein gleichwertiger Erfolg der als Kombinationstherapie verabreichten RIT gegenüber einer leitliniengerechten Therapie gezeigt wurde.

Wie bereits im Voraus beschrieben (siehe Kapitel 4.1.2, S.66), sind die Registerauswertungen bezüglich der Überlebenszeiten und Langzeitergebnisse besonders im dritten Nachuntersuchungsjahr nicht ausreichend aussagekräftig und valide, sodass der Vergleich des 3-Jahres-OS und -PFS zwischen Registerpatienten und der MInT-Studie nur zur Vollständigkeit dokumentiert wurde. Auf Grund der mangelnden Aussagekraft können an dieser Stelle keine prognostischen Angaben zum Langzeitverlauf der RIT im Vergleich zu einer konventionellen Polychemotherapie in Kombination mit Rituximab gemacht werden.

Insgesamt konnte gezeigt werden, dass die zusätzliche, zumeist als Konsolidierung angewandte RIT der alleinigen konventionellen Standardtherapie nicht unterlegen ist, sondern eine neue, mindestens vergleichbar erfolgreiche Therapieoption darstellt. 


\subsection{Chancen der Radioimmuntherapie}

Zusammenfassend konnte in der vorliegenden Arbeit anhand eines relativ großen Patientenkollektivs, welches die Patienten der alltäglichen Klinikroutine in ihren Eigenschaften repräsentiert, die Wirksamkeit und Durchführbarkeit von RIT bei DLBCL gezeigt werden. Durch die große Variationsbreite der Registerpatienten bezüglich Alter, Lymphomausprägung, Risiko- und Prognoseeinschätzung sowie Indikationsstellung war nicht immer ein direkter Vergleich zu den Patientenkollektiven der ausschlaggebenden klinischen Studien über Therapiestrategien bei DLBCL möglich, da diese meist kleiner sind und zudem genau definierten Einschlusskriterien entsprechen. Dennoch konnte gezeigt werden, dass der Therapieerfolg, welcher im RIT-Register erreicht wurde und auch trotz gelegentlicher Herausforderungen in der Erhebung der Registerdaten den wirklichen Therapieerfolg im klinischen Alltag gut widerspiegelt, mindestens ebenso erfolgversprechend wie der anderer maßgeblicher Studien zur Anwendung der RIT bei DLBCL (siehe Kapitel 4.2, S.70) ist. Die RIT ist also auch außerhalb kontrollierter klinischer Studien und unter Alltagsbedingungen effizient und wirkungsvoll bei tolerablen unerwünschten Nebenwirkungen durchführbar. Es zeigt sich zudem, dass die zumeist mit vorangegangener Chemotherapie kombinierte RIT in Abhängigkeit der Indikationsstellung bessere Therapieerfolge erzielt. Die "First line"Patienten, welche zuvor unbehandelt waren, erreichten erwartungsgemäß signifikant bessere Ansprechraten und Überlebenszeiten als das "Relapse"-Patientenkollektiv, welches die RIT als Zweitlinientherapie oder mehr erhalten hatte.

$\mathrm{Da}$, wie sich durch die kurze mediane Nachuntersuchungszeit zeigt, zu wenige "patients at risk" nach 24 oder sogar 36 Monaten untersucht wurden und die Aussagekraft der Langzeitauswertungen infolgedessen im zweiten und besonders im dritten Nachbeobachtungsjahr limitiert sind, war diesbezüglich kein ausdrucksstarker Vergleich möglich. Eine erneute Auswertung des RIT-Registers in einigen Jahren sollte jedoch die mediane Nachuntersuchungszeit anheben und möglicherweise Langzeitdaten mit größerem Informationswert hervorbringen. Patienten, für die bis zum Ende der Datenerhebung der vorliegenden Arbeit beispielsweise erst eine 12-Monats-Verlaufskontrolle dokumentiert wurde, könnten dann schon bis zu 36 Monate nachuntersucht und dokumentiert worden sein.

Um die Wirksamkeit der RIT nicht nur in Abhängigkeit der Indikationsstellung zu zeigen, sondern auch eine eventuelle Abhängigkeit von Patientenalter, IPI und Rituximab als Therapiebestandteil zu untersuchen, sollte auch eine erneute Analyse des "First line"Patientenkollektivs in Hinblick auf diese Eigenschaften in einiger Zeit erfolgen. Obwohl zu 
erwarten gewesen wäre, dass die Patienten ein unterschiedliches Langzeitverhalten nach RIT in Abhängigkeit dieser Parameter aufweisen, konnte dies in der vorliegenden Arbeit nicht eindeutig gezeigt werden. Aus bereits diskutierten Gründen besteht hier jedoch eine große Unsicherheit über die Validität der Auswertung, so dass eine erneute Auswertung, sobald mehr Langzeitdaten vorliegen, sinnvoll erscheint. Möglicherweise wäre auch eine größere, randomisierte und kontrollierte Studie über die Wirksamkeit von RIT in Abhängigkeit von Patientenalter, IPI und Rituximab hilfreich, um das Wirksamkeitsprofil der RIT bei DLBCL besser einordnen zu können.

Im Vergleich mit den verschiedenen Studien, welche die Etablierung einer Polychemotherapie in Kombination mit Rituximab in den aktuellen Therapieleitlinien zur Folge hatten, konnte sich durch die guten Therapieergebnisse der Registeranalyse sowohl im Gesamtkollektiv als auch im "First line"-Kollektiv die RIT als neue Therapieoption in der Behandlung von DLBCL behaupten (siehe Kapitel 4.3, S.81). Besonders bei älteren Patienten (>60 Jahre) erzielte die RIT in Erstlinientherapie vergleichbar gute Erfolge wie eine konventionelle Therapie. Im Langzeitverlauf zeigte sich hier sogar eine Überlegenheit der RIT nach zwei Jahren.

Aber auch das Gesamtkollektiv zeigte ein gutes Therapieansprechen (ORR $=63,3 \%)$, so dass die RIT ebenfalls eine gute Wirksamkeit in einer gemischten Patientengruppe zeigt, welche ein heterogenes Maß an Vortherapien und Indikationsstellungen aufweist. Die Behandlung rezidivierter und therapierefraktärer DLBCL, welche bislang noch unbefriedigend ist, könnte folglich durch eine Anwendung von RIT längerfristig verbessert werden. Auch hier wäre jedoch eine klinische Untersuchung der Wirksamkeit von RIT bei mehrfach rezidivierten und therapierefraktären Lymphomen als randomisierte und kontrollierte Studie sinnvoll.

Desgleichen könnte eine RIT auch für jüngere Hochrisikopatienten neue Erfolge in der Therapie erbringen. Hier besteht bis dato kein allgemein gültiger Therapiestandard, da noch kein deutlich überlegenes und erfolgreiches Therapieprotokoll gefunden wurde (Tilly und Dreyling 2010). Eine klinische Studie über die Anwendung von RIT im Vergleich mit einer Polychemotherapie und Rituximab in dieser Patientengruppe erscheint somit empfehlenswert.

Zusammenfassend zeigen die Ergebnisse der Registeranalyse, dass die RIT auch im klinischen Alltag sicher und effizient angewandt werden kann und eine neue Therapieoption in der Behandlung von DLBCL darstellt. Hier ist besonders die Möglichkeit der erfolgreichen Behandlung rezidivierter oder therapierefraktärer Lymphome, bei denen die herkömmlichen Therapiestandards nicht zum Erfolg führen, zu nennen. Es sind jedoch noch weitere Studien 
nötig, um den Langzeitverlauf der RIT sowie deren Wirksamkeit in Abhängigkeit von Patientenalter, IPI und Rituximab als Bestandteil der Vortherapie besser einschätzen zu können.

Die vorliegende Arbeit zeigt, dass weitere Untersuchungen zur Anwendung von RIT bei DLBCL vonnöten, jedoch auch sinnvoll und vielversprechend sind, um letztendlich eine Zulassung von RIT bei DLBCL zu erreichen.

\subsubsection{Offene Fragen in der Anwendung von RIT bei DLBCL}

Wie bereits diskutiert wurde, besteht die Notwendigkeit, eine RIT bei DLBCL bezüglich verschiedener Eigenschaften weitergehend zu untersuchen. Dazu gehören der Langzeitverlauf und die Langzeitwirksamkeit der Therapie sowie ihre Anwendbarkeit in Abhängigkeit von Patientenalter, IPI und Rituximab als Bestandteil der Vortherapie. Ebenso sollte die Anwendung von RIT bei mehrfach rezidivierten oder therapierefraktären Lymphomen und bei jüngeren Hochrisikopatienten analysiert werden.

Es bestehen jedoch auch weitere offene Fragen in der Anwendung von RIT bei DLBCL welche in der Auswertung der vorliegenden Arbeit nicht untersucht und hier nicht eingehend diskutiert wurden, jedoch Bestandteil aktueller und zukünftiger Forschung sind:

So sollte beispielsweise untersucht werden, ob Patienten mit einer PR nach leitliniengerechter Therapie durch eine anschließende RIT zu einer CR konvertieren würden und somit durch die RIT einen Vorteil erzielen. Des Weiteren ist die Anwendung und Wirksamkeit von RIT bei großen Tumormassen, welche im Allgemeinen "Bulky Disease" genannt werden, Bestandteil aktueller Studien. Ebenso bestehen Unsicherheiten, ob die RIT eine Strahlentherapie als Konsolidierung in der Behandlung maligner Lymphome ersetzen kann. Bei kommenden Untersuchungen zur Anwendung von RIT bei mehrfach rezidivierten DLBCL sollte zudem ermittelt werden, ob die RIT alleine oder in Kombination mit einer HDC (beispielsweise Z-BEAM) mehr Wirksamkeit und Sicherheit bietet. 


\section{Zusammenfassung}

Die vorliegenden Arbeit zeigt, dass eine Radioimmuntherapie (RIT) in der klinischen Routine sicher und effizient bei diffus großzelligen B-Zell-Lymphomen (DLBCL) angewandt werden kann und neue Chancen in der Behandlung jüngerer Hochrisikopatienten sowie rezidivierter und therapierefraktärer Lymphome bietet.

Die aktuellen Therapieleitlinien zur Behandlung maligner Lymphome empfehlen derzeit bei DLBCL eine Kombinationstherapie aus dem Polychemotherapie-Protokoll CHOP und dem CD20-Antikörper Rituximab. In Abhängigkeit des Patientenalters und des individuellen Risikos, welches anhand des Internationalen Prognostischen Index (IPI) ermittelt wird, sollen verschiede Applikationsschemata eingehalten werden. Demzufolge werden Patienten, die mindestens 60 Jahre alt sind, mit sechs Zyklen CHOP sowie acht Zyklen Rituximab im zweiwöchigen Abstand (6 x CHOP-14 + 8 x R) beziehungsweise mit acht Zyklen R-CHOP alle drei Wochen $(8 \times$ R-CHOP-21) behandelt. Patienten unter 60 Jahren mit einem niedrigen oder niedrigintermediärem Risiko (IPI $\leq 2)$ sollten dagegen als Standardtherapie sechs Zyklen R-CHOP-21 erhalten. Für jüngere Patienten $(<60 \mathrm{Jahre})$, die einen IPI größer als zwei aufweisen, kann dagegen zur Zeit noch keine eindeutige Therapieempfehlung gegeben werden, obwohl kürzlich die bis dato besten Therapieergebnisse mittels acht Zyklen CHOEP im zweiwöchentlichen Abstand in Kombination mit sechs Zyklen Rituximab (8 x CHOEP-14 + 6 x R-14) erzielt wurden.

Schwächen der konventionellen Immun-Chemotherapie bestehen folglich in der Behandlung jüngerer Hochrisikopatienten sowie in der Anwendung bei therapierefraktären und rezidivierten Lymphomen, auf Grund dessen hier nach neuen Therapieoptionen gesucht wird. Eine solche moderne Option stellt die RIT als kombinierte, systemische Immun- und Strahlentherapie dar. In der vorliegenden Arbeit wurde ihre Durchführbarkeit und Sicherheit bei Patienten mit DLBCL außerhalb klinischer Studien untersucht und bewertet.

Die Auswertung von 215 Patientendaten, welche in einem internationalen internetbasierten Register dokumentiert und gespeichert wurden, zeigt, dass eine RIT bei tolerablen Nebenwirkungen effektiv und erfolgsversprechend in der Behandlung von DLBCL ist. Das Gesamtkollektiv weist eine Gesamtansprechrate (ORR) von 63,3\% mit einem Anteil von 54,4 \% kompletten Remissionen (CR) auf, während das "First line"-Patientenkollektiv sogar eine ORR von 83,3\% mit 76,4\% CR erreicht. Es konnte somit gezeigt werden, dass DLBCLPatienten davon profitieren, die RIT als Erstlinientherapie mit der Indikation zur Konsolidierung, als Konditionierung im Rahmen einer Hochdosischemotherapie oder als 
primäre Therapie zu erhalten. Dies wird zudem im signifikant besseren Gesamt- und progressfreien Überleben sowie der signifikant längeren progressfreien Zeit der "First line"Patienten gegenüber den "Relapse"-Patienten ( $\mathrm{p}$-Werte $=0,0071 ; 0,018$ und 0,0056) deutlich. Da auch das Gesamtkollektiv ein gutes Therapieansprechen zeigt, obwohl hier bereits vielfache Vortherapien, ein heterogenes Risikoprofil sowie mehrfach rezidivierte beziehungsweise therapierefraktäre Lymphome vorliegen, bietet die RIT auch hier neue Erfolgschancen. Im Vergleich der in der vorliegenden Arbeit erzielten Ergebnisse mit den Ergebnissen der zentralen Studien zur Untersuchung einer RIT bei DLBCL wird deutlich, dass die RIT auch in der klinischen Routine mindestens gleichermaßen erfolgsversprechend und durchführbar wie im Kontext randomisierter Studien ist.

Weiterhin zeigt ein Vergleich der zur Etablierung der Therapieleitlinien maßgeblichen Studien mit der Registeranalyse, dass eine RIT dem konventionellen Therapiestandard nicht unterlegen ist. Sie bietet dagegen neue Möglichkeiten für die Behandlung von DLBCLPatienten, bei denen eine leitliniengerechte Therapie nicht zu einer langfristigen Remission und Progressfreiheit führt.

Trotz der Limitationen, welche sich aus einer Registerauswertung ergeben, und den mangelnden Langzeitdaten, welche durch einen zu kurzen Datenerhebungszeitraum bedingt sein können, konnte in der vorliegenden Arbeit die Anwendung von RIT bei DLBCL in der klinischen Routine ohne Selektion des Patientenkollektivs wiedergegeben werden. Um jedoch auch den Langzeitverlauf der RIT sowie deren Wirksamkeit in Abhängigkeit von Patientenalter, dem IPI sowie den Bestandteilen der Vortherapie besser einschätzen zu können, sind hier noch weitere, zukünftige Auswertungen des RIT-Registers oder gegebenenfalls weitere randomisierte, kontrollierte Studien vonnöten.

Die Ergebnisse der Registeranalyse weisen somit darauf hin, dass die RIT bei DLBCL eine neue, sichere und effiziente Therapieoption, insbesondere als Erstlinientherapie, darstellt. Des Weiteren können die Behandlungserfolge jüngerer Hochrisikopatienten sowie rezidivierter oder therapierefraktärer DLBCL durch eine RIT maßgeblich verbessert werden. Gemeinsam mit bereits bestehenden sowie kommenden Studien tragen die Ergebnisse der vorliegenden Arbeit unterstützend dazu bei, dass eine Zulassung der RIT bei DLBCL sowie die Aufnahme der RIT in die Leitlinien zur Behandlung bei DLBCL nach erfolgter Prüfung mittels einer Phase-III-Studie in Betracht gezogen werden sollte. 


\section{Literaturverzeichnis}

Aviles A, Fernandezb R, Perez F, Nambo MJ, Neri N, Talavera A, Castaneda C, Gonzalez M, Cleto S (2004): "Adjuvant radiotherapy in stage IV diffuse large cell lymphoma improves outcome." Leuk Lymphoma 45(7): 1385-1389.

Carbone PP, Kaplan HS, Musshoff K, Smithers DW, Tubiana M (1971): "Report of the Committee on Hodgkin's Disease Staging Classification." Cancer Res 31(11): 18601861.

Chapuy B, Borchmann P, Engert A, Trümper L (2006): "Targeted-Therapie: Neue Zielstrukturen und Substanzen in der Behandlung maligner Lymphome." Der Onkologe 12: 651-658.

Chapuy B, Hohloch K, Trümper L (2007): "Yttrium 90 ibritumomab tiuxetan (Zevalin): a new bullet in the fight against malignant lymphoma?" Biotechnol J 2(11): 1435-1443.

Coiffier B, Lepage E, Briere J, Herbrecht R, Tilly H, Bouabdallah R, Morel P, Van Den Neste E, Salles G, Gaulard P et al. (2002): "CHOP chemotherapy plus rituximab compared with CHOP alone in elderly patients with diffuse large-B-cell lymphoma." N Engl J Med 346(4): 235-242.

Coiffier B, Thieblemont C, Van Den Neste E, Lepeu G, Plantier I, Castaigne S, Lefort S, Marit G, Macro M, Sebban C et al. (2010): "Long-term outcome of patients in the LNH-98.5 trial, the first randomized study comparing rituximab-CHOP to standard CHOP chemotherapy in DLBCL patients: a study by the Groupe d'Etudes des Lymphomes de l'Adulte." Blood 116(12): 2040-2045.

Czuczman MS, Emmanouilides C, Darif M, Witzig TE, Gordon LI, Revell S, Vo K, Molina A (2007): "Treatment-related myelodysplastic syndrome and acute myelogenous leukemia in patients treated with ibritumomab tiuxetan radioimmunotherapy." J Clin Oncol 25(27): 4285-4292.

Devizzi L, Guidetti A, Tarella C, Magni M, Matteucci P, Seregni E, Chiesa C, Bombardieri E, Di Nicola M, Carlo-Stella C et al. (2008): "High-dose yttrium-90-ibritumomab tiuxetan with tandem stem-cell reinfusion: an outpatient preparative regimen for autologous hematopoietic cell transplantation." J Clin Oncol 26 (32): 5175-5182.

Emmanouilides C (2007): "Radioimmunotherapy for non-hodgkin lymphoma: historical perspective and current status." J Clin Exp Hematop 47(2): 43-60.

Emmanouilides C, Witzig TE, Wiseman GA, Gordon LI, Wang H, Schilder R, Saville MW, Flinn I, Molina A (2007): "Safety and efficacy of yttrium-90 ibritumomab tiuxetan in older patients with non-Hodgkin's lymphoma." Cancer Biother Radiopharm 22(5): 684-691.

Fisher RI, Gaynor ER, Dahlberg S, Oken MM, Grogan TM, Mize EM, Glick JH, Coltman CA, Jr. Miller TP (1993): "Comparison of a standard regimen (CHOP) with three intensive chemotherapy regimens for advanced non-Hodgkin's lymphoma." N Engl J Med 328(14): 1002-1006.

Gisselbrecht C, Lepage E, Molina T, Quesnel B, Fillet G, Lederlin P, Coiffier B, Tilly H, Gabarre J, Guilmin F et al. (2002): "Shortened first-line high-dose chemotherapy for patients with poor-prognosis aggressive lymphoma." J Clin Oncol 20(10): 2472-2479.

Gisselbrecht C, Vose J, Nademanee A, Gianni AM, Nagler A (2009): "Radioimmunotherapy for stem cell transplantation in non-Hodgkin's lymphoma: in pursuit of a complete response." Oncologist 14 Suppl 2: 41-51. 
Gisselbrecht C, Glass B, Mounier N, Singh Gill D, Linch DC, Trneny M, Bosly A, Ketterer N, Shpilberg O, Hagberg $\mathrm{H}$ et al. (2010): "Salvage regimens with autologous transplantation for relapsed large B-cell lymphoma in the rituximab era." J Clin Oncol 28(27): 4184-4190.

Glass B, Ziepert M, Reiser M, Freund M, Trümper L, Metzner B, Feller A, Loeffler M, Pfreundschuh M, Schmitz N (2010): "High-dose therapy followed by autologous stem-cell transplantation with and without rituximab for primary treatment of highrisk diffuse large B-cell lymphoma." Ann Oncol 21(11): 2255-2261.

Gordon LI, Molina A, Witzig T, Emmanouilides C, Raubtischek A, Darif M, Schilder RJ, Wiseman G, White CA (2004): "Durable responses after ibritumomab tiuxetan radioimmunotherapy for $\mathrm{CD} 20+\mathrm{B}$-cell lymphoma: long-term follow-up of a phase $1 / 2$ study." Blood 103(12): 4429-4431.

Gregory SA, Hohloch K, Gisselbrecht C, Tobinai K, Dreyling M (2009): "Harnessing the energy: development of radioimmunotherapy for patients with non-Hodgkin's lymphoma." Oncologist 14 Suppl 2: 4-16.

Haioun C, Lepage E, Gisselbrecht C, Salles G, Coiffier B, Brice P, Bosly A, Morel P, Nouvel C, Tilly $\mathrm{H}$ et al. (2000): "Survival benefit of high-dose therapy in poor-risk aggressive non-Hodgkin's lymphoma: final analysis of the prospective LNH87-2 protocol--a groupe d'Etude des lymphomes de l'Adulte study." J Clin Oncol 18(16): 3025-3030.

Harris NL, Jaffe ES, Stein H, Banks PM, Chan JK, Cleary ML, Delsol G, De Wolf-Peeters C, Falini B, Gatter KC et al. (1994): "A revised European-American classification of lymphoid neoplasms: a proposal from the International Lymphoma Study Group." Blood 84(5): 1361-1392.

Held G, Murawski N, Pfreundschuh M (2011): "Therapiestrategien beim diffusen großzelligen B-Zell-Lymphom." Der Onkologe 17: 789 - 798.

Illidge T, Tolan S (2008): "Current treatment approaches for diffuse large B-cell lymphoma." Leuk Lymphoma 49(4): 663-676.

Jacobs SA, Vidnovic N, Joyce J, McCook B, Torok F, Avril N (2005): "Full-dose 90Y ibritumomab tiuxetan therapy is safe in patients with prior myeloablative chemotherapy." Clin Cancer Res 11(19 Pt 2): 7146s-7150s.

Jaffe ES (2009): "The 2008 WHO classification of lymphomas: implications for clinical practice and translational research." Hematol Am Soc Hematol Educ Program: 523531.

Kaiser U, Uebelacker I, Abel U, Birkmann J, Trümper L, Schmalenberg H, Karakas T, Metzner B, Hossfeld DK, Bischoff HG et al. (2002): "Randomized study to evaluate the use of high-dose therapy as part of primary treatment for "aggressive" lymphoma." J Clin Oncol 20(22): 4413-4419.

Kaminski MS, Zasadny KR, Francis IR, Milik AW, Ross CW, Moon SD, Crawford SM, Burgess JM, Petry NA, Butchko GM et al. (1993): "Radioimmunotherapy of B-cell lymphoma with [131I]anti-B1 (anti-CD20) antibody." N Engl J Med 329(7): 459-465.

Krishnan A, Nademanee A, Fung HC, Raubitschek AA, Molina A, Yamauchi D, Rodriguez R, Spielberger RT, Falk P, Palmer JM et al. (2008): "Phase II trial of a transplantation regimen of yttrium-90 ibritumomab tiuxetan and high-dose chemotherapy in patients with non-Hodgkin's lymphoma." J Clin Oncol 26(1): 90-95.

Lennert K, Stein H, Kaiserling E (1975): "Cytological and functional criteria for the classification of malignant lymphomata." Br J Cancer Suppl 2: 29-43.

Morschhauser F, Illidge T, Huglo D, Martinelli G, Paganelli G, Zinzani PL, Rule S, Liberati AM, Milpied N, Hess G et al. (2007): "Efficacy and safety of yttrium-90 ibritumomab tiuxetan in patients with relapsed or refractory diffuse large B-cell lymphoma not appropriate for autologous stem-cell transplantation." Blood 110(1): 54-58. 
Morschhauser F, Radford J, Van Hoof A, Vitolo U, Soubeyran P, Tilly H, Huijgens PC, Kolstad A, d'Amore F, Gonzalez Diaz M et al. (2008): "Phase III trial of consolidation therapy with yttrium-90-ibritumomab tiuxetan compared with no additional therapy after first remission in advanced follicular lymphoma." J Clin Oncol 26(32): 51565164.

Moser EC, Kluin-Nelemans HC, Carde P, Meerwaldt JH, Tirelli U, Aleman BM, Baars J, Thomas J, van Glabbeke M, Noordijk EM (2006): "Impact of involved field radiotherapy in partial response after doxorubicin-based chemotherapy for advanced aggressive non-Hodgkin's lymphoma." Int J Radiat Oncol Biol Phys 66(4): 11681177.

Nademanee A, Forman S, Molina A, Fung H, Smith D, Dagis A, Kwok C, Yamauchi D, Anderson AL, Falk P et al. (2005): "A phase 1/2 trial of high-dose yttrium-90ibritumomab tiuxetan in combination with high-dose etoposide and cyclophosphamide followed by autologous stem cell transplantation in patients with poor-risk or relapsed non-Hodgkin lymphoma." Blood 106(8): 2896-2902.

Pfreundschuh M, Trümper L, Kloess M, Schmits R, Feller AC, Rube C, Rudolph C, Reiser M, Hossfeld DK, Eimermacher H et al. (2004a): "Two-weekly or 3-weekly CHOP chemotherapy with or without etoposide for the treatment of elderly patients with aggressive lymphomas: results of the NHL-B2 trial of the DSHNHL." Blood 104(3): 634-641.

Pfreundschuh M, Trümper L, Kloess M, Schmits R, Feller AC, Rudolph C, Reiser M, Hossfeld DK, Metzner B, Hasenclever D et al. (2004b): "Two-weekly or 3-weekly CHOP chemotherapy with or without etoposide for the treatment of young patients with good-prognosis (normal LDH) aggressive lymphomas: results of the NHL-B1 trial of the DSHNHL." Blood 104(3): 626-633.

Pfreundschuh M, Trümper L, Osterborg A, Pettengell R, Trneny M, Imrie K, Ma D, Gill D, Walewski J, Zinzani PL et al. (2006): "CHOP-like chemotherapy plus rituximab versus CHOP-like chemotherapy alone in young patients with good-prognosis diffuse large-B-cell lymphoma: a randomised controlled trial by the MabThera International Trial (MInT) Group." Lancet Oncol 7(5): 379-391.

Pfreundschuh M, Ho AD, Cavallin-Stahl E, Wolf M, Pettengell R, Vasova I, Belch A, Walewski J, Zinzani PL, Mingrone W et al. (2008a): "Prognostic significance of maximum tumour (bulk) diameter in young patients with good-prognosis diffuse large-B-cell lymphoma treated with CHOP-like chemotherapy with or without rituximab: an exploratory analysis of the MabThera International Trial Group (MInT) study." Lancet Oncol 9 (5): 435-444.

Pfreundschuh M, Schubert J, Ziepert M, Schmits R, Mohren M, Lengfelder E, Reiser M, Nickenig C, Clemens M, Peter N et al. (2008b): "Six versus eight cycles of bi-weekly CHOP-14 with or without rituximab in elderly patients with aggressive CD20+ B-cell lymphomas: a randomised controlled trial (RICOVER-60)." Lancet Oncol 92(2): 105116.

Pfreundschuh M, Kuhnt E, Trümper L, Osterborg A, Trneny M, Shepherd L, Gill DS, Walewski J, Pettengell R, Jaeger U et al. (2011): "CHOP-like chemotherapy with or without rituximab in young patients with good-prognosis diffuse large-B-cell lymphoma: 6-year results of an open-label randomised study of the MabThera International Trial (MInT) Group." Lancet Oncol 12(11): 1013-1022.

Philip T, Guglielmi C, Hagenbeek A, Somers R, Van der Lelie H, Bron D, Sonneveld P, Gisselbrecht C, Cahn JY, Harousseau JL et al. (1995): "Autologous bone marrow transplantation as compared with salvage chemotherapy in relapses of chemotherapysensitive non-Hodgkin's lymphoma." N Engl J Med 333(23): 1540-1545. 
Press OW, Eary JF, Appelbaum FR, Martin PJ, Badger CC, Nelp WB, Glenn S, Butchko G, Fisher D, Porter B et al. (1993): "Radiolabeled-antibody therapy of B-cell lymphoma with autologous bone marrow support." N Engl J Med 329(17): 1219-1224.

Reyes F, Lepage E, Ganem G, Molina TJ, Brice P, Coiffier B, Morel P, Ferme C, Bosly A, Lederlin $\mathrm{P}$ et al. (2005): "ACVBP versus CHOP plus radiotherapy for localized aggressive lymphoma." N Engl J Med 352(12): 1197-1205.

Rosenberg SA, Berard CW, Brown BWJ, Burke J, Dorfman RF, Glatstein E, Hoppe RT, Simon R (1982): "National Cancer Institute sponsored study of classifications of nonHodgkin's lymphomas: summary and description of a working formulation for clinical usage. The Non-Hodgkin's Lymphoma Pathologic Classification Project." Cancer 49(10): 2112-2135.

Schmitz N, Kloess M, Reiser M, Berdel WE, Metzner B, Dorken B, Kneba M, Trümper L, Loeffler M, Pfreundschuh M et al. (2006): "Four versus six courses of a doseescalated cyclophosphamide, doxorubicin, vincristine, and prednisone (CHOP) regimen plus etoposide (megaCHOEP) and autologous stem cell transplantation: early dose intensity is crucial in treating younger patients with poor prognosis aggressive lymphoma." Cancer 106(1): 136-145.

Schmitz N, Nickelsen M, Ziepert M, Haenel M, Borchmann P, Schmidt C, Viardot A, Bentz M, Peter N, Ehninger G et al. (2011): "Conventional chemoimmunotherapy (RCHOEP-14) or high-dose therapy (R-Mega-CHOEP) for young, high-risk patients with aggressive B-cell lymphoma: Final results of the randomized Mega-CHOEP trial of the German High-Grade Non-Hodgkin Lymphona Study Group (DSHNHL)." J Clin Oncol 29 Suppl 15: 8002.

Shimabukuro-Vornhagen A, Josting A, Hübel K, Scheid K, Dietlein M, Engert A, Schnell R (2008): "Yttrium-90 ibritumomab tiuxetan combined with high-dose BEAM chemotherapy and autologous stem cell transplantation for relapsed/refractory B-cell non-Hodgkin's lymphoma." J Clin Oncol 26(15suppl): Abstract 8615.

Shimoni A, Zwas ST, Oksman Y, Hardan I, Shem-Tov N, Yerushalmi R, Avigdor A, BenBassat I, Nagler A (2007): "Yttrium-90-ibritumomab tiuxetan (Zevalin) combined with high-dose BEAM chemotherapy and autologous stem cell transplantation for chemo-refractory aggressive non-Hodgkin's lymphoma." Exp Hematol 35(4): 534540.

Shipp MD, Harrington DP, Anderson JR, Armitage JO, Bonadonna G, Brittinger G, Cabanillas F, Canellos GP, Coiffier B, Connors JM (1993): "A predictive model for aggressive non-Hodgkin's lymphoma. The International Non-Hodgkin's Lymphoma Prognostic Factors Project." N Engl J Med 329(14): 987-994.

Spicer J, Smith P, Maclennan K, Hoskin P, Hancock B, Linch D, Pettengell R (2004): "Longterm follow-up of patients treated with radiotherapy alone for early-stage histologically aggressive non-Hodgkin's lymphoma." Br J Cancer 90(6): 1151-1155.

Stashenko P, Nadler LM, Hardy R, Schlossman SF (1980): "Characterization of a human B lymphocyte-specific antigen." J Immunol 125(4): 1678-1685.

Thieblemont C, Briere J, Mounier N, Voelker HU, Cuccuini W, Hirchaud E, Rosenwald A, Jack A, Sundstorm C, Cogliatti S et al. (2011): "The Germinal Center/Activated BCell Subclassification Has a Prognostic Impact for Response to Salvage Therapy in Relapsed/Refractory Diffuse Large B-Cell Lymphoma: A Bio-CORAL Study." J Clin Oncol, im Druck.

Tiemann M, Trümper L (2001): "Pathologie, Biologie und Klassifikaton maligner NonHodgkin-Lymphome." Der Onkologe 7(9): 940-959.

Tilly H, Dreyling M (2010): "Diffuse large B-cell non-Hodgkin's lymphoma: ESMO Clinical Practice Guidelines for diagnosis, treatment and follow-up." Ann Oncol 21 Suppl 5: v172-174. 
Trümper L, Bauer S, Blumstein N, Buchegger F, Bunjes D, Bischof-Delaloye A, Dietlein M, Engert A, Friesen C, Glass B et al.: Radioimmuntherapie in der Hämatologie und Onkologie. 1.Auflage; UNI-MED Verlag AG, Bremen 2007.

Vose JM (2004): "Bexxar: novel radioimmunotherapy for the treatment of low-grade and transformed low-grade non-Hodgkin's lymphoma." Oncologist 9 (2): 160-172.

Vose JM, Bierman PJ, Loberiza FR, Bociek RG, Matso D, Armitage JO (2007): "Phase I trial of (90)Y-ibritumomab tiuxetan in patients with relapsed B-cell non-Hodgkin's lymphoma following high-dose chemotherapy and autologous stem cell transplantation." Leuk Lymphoma $\underline{48(4): ~ 683-690 . ~}$

Witzig TE, Gordon LI, Cabanillas F, Czuczman MS, Emmanouilides C, Joyce R, Pohlman BL, Bartlett NL, Wiseman GA, Padre N et al. (2002): "Randomized controlled trial of yttrium-90-labeled ibritumomab tiuxetan radioimmunotherapy versus rituximab immunotherapy for patients with relapsed or refractory low-grade, follicular, or transformed B-cell non-Hodgkin's lymphoma." J Clin Oncol 20(10): 2453-2463.

Witzig TE, White CA, Gordon LI, Wiseman GA, Emmanouilides C, Murray JL, Lister J, Multani PS (2003): "Safety of yttrium-90 ibritumomab tiuxetan radioimmunotherapy for relapsed low-grade, follicular, or transformed non-hodgkin's lymphoma." J Clin Oncol 21(7): 1263-1270.

Zinzani PL, Tani M, Fanti S, Stefoni V, Musuraca G, Castellucci P, Marchi E, Farsad M, Fina M, Pellegrini C et al. (2008): "A phase II trial of CHOP chemotherapy followed by yttrium 90 ibritumomab tiuxetan (Zevalin) for previously untreated elderly diffuse large B-cell lymphoma patients." Ann Oncol 19(4): 769-773.

Zinzani PL, Rossi G, Franceschetti S, Botto B, Di Rocco A, Cabras MG, Petti MC, Stefoni V, Broccoli A, Fanti S et al. (2010): "Phase II trial of short-course R-CHOP followed by 90Y-ibritumomab tiuxetan in previously untreated high-risk elderly diffuse large Bcell lymphoma patients." Clin Cancer Res 16(15): 3998-4004. 


\section{Anhang}

\subsection{Abkürzungsverzeichnis}

\begin{tabular}{|c|c|}
\hline${ }^{131} \mathrm{I}$ & ${ }^{131} \operatorname{Iod}$ \\
\hline${ }^{131} \mathrm{I}-\mathrm{T}$ & ${ }^{131}$ Iod-Tositumomab \\
\hline${ }^{90} \mathrm{Y}$ & ${ }^{90}$ Yttrium \\
\hline${ }^{90} \mathrm{Y}-\mathrm{IT}$ & ${ }^{90}$ Yttrium-Ibritumomab Tiuxetan \\
\hline aaIPI & altersadaptierter International Prognostischer Index \\
\hline ACBVP & $\begin{array}{l}\left.\text { Doxorubicin (Adriblastin }{ }^{\circledR}\right) \text {, Cyclophosphamid, Bleomycin, Vindesin, } \\
\text { Prednison }\end{array}$ \\
\hline allo SZT & allogene Stammzelltransplantation \\
\hline AML & akute myeloische Leukämie \\
\hline ASCO & American Society of Clinical Oncology \\
\hline autoSZT & autologe Stammzelltransplantation \\
\hline BEAM & Carmustin (BCNU®), Etoposid, Cytarabinosid (Alexan®), Melphalan \\
\hline $\mathrm{CD}$ & Cluster of Differentiation \\
\hline CHOEP & $\begin{array}{l}\text { Cyclophosphamid, Doxorubicin (Hydroxyldaunorubicin), } \\
\text { Vincristin (Oncovin®), Etoposid, Prednison }\end{array}$ \\
\hline $\mathrm{CHOP}$ & $\begin{array}{l}\text { Cyclophosphamid, Doxorubicin (Hydroxyldaunorubicin), } \\
\text { Vincristin (Oncovin } ®), \text { Prednison }\end{array}$ \\
\hline CHOP-14 & $\begin{array}{l}\text { Cyclophosphamid, Doxorubicin (Hydroxyldaunorubicin), } \\
\text { Vincristin (Oncovin } ®), \text { Prednison alle } 14 \text { Tage }\end{array}$ \\
\hline CHOP-21 & $\begin{array}{l}\text { Cyclophosphamid, Doxorubicin (Hydroxyldaunorubicin), } \\
\text { Vincristin (Oncovin } ®), \text { Prednison alle } 21 \text { Tage }\end{array}$ \\
\hline CNOP & $\begin{array}{l}\left.\text { Cyclophosphamid, Mitoxantron (Novatron }{ }^{\circledR}\right) \text {, } \\
\text { Vincristin (Oncovin®), Prednison }\end{array}$ \\
\hline $\mathrm{COP}$ & Cyclophosphamid, Vincristin (Oncovin $\left.{ }^{\circledR}\right)$, Prednison \\
\hline
\end{tabular}


$\mathrm{CRu}$

DHAP

DLBCL

DSHNHL

ECOG

ESHAP

FL

FOP

GELA

$\mathrm{GmbH}$

$\mathrm{Hb}$

HDC

IPI

$\mathrm{LDH}$

MALT

MDS

MInT

MTX

n.d.

NHL

NK-Zellen

ORR

OS

PD

PFS complete remission unconfirmed - unbestätigte komplette Remission

Dexamethason, Cytarabin (Ara C), Cisplatin

Diffuse Large B-Cell Lymphoma - diffus großzellige B-Zell-Lymphome

Deutsche Studiengruppe Hochmaligne Non-Hodgkin-Lymphome

Eastern Cooperative Oncology Group

Etoposid, Methylprednisolon, Cytarabin (Ara C), Cisplatin

Follikuläre Lymphome

Fludarabin, Vincristin (Oncovin®),Prednison

Groupe d'Etude des Lymphomes d'Adulte

Gesellschaft mit beschränkter Haftung

Hämoglobin

Hochdosis-Chemotherapie

Internationaler Prognostischer Index

Laktatdehydrogenase

Mucosa Associated Lymphoid Tissue -

Schleimhaut-assoziiertes lymphatisches Gewebe

myelodysplastisches Syndrom

MabThera International Trial

Methotrexat

nicht dokumentiert

Non-Hodgkin-Lymphome

Natürliche Killer-Zellen

overall response rate - Gesamtansprechrate

overall survival - Gesamtüberleben

progressive disease - Progress

progression-free survival - progressfreies Überleben 
PR

$\mathrm{R}$

R.E.A.L.-

Klassifikation

RIT Radioimmuntherapie

SAS Statistical Analysis Systems

SD

TTP

USA

WHO

Z-BEAM

partielle Remission

Rituximab
Revised European-American Classification of Lymphoid Neoplasms

stable disease - stabile Erkrankung

time to progression - progressfreie Zeit

United States of America - Vereinigte Staaten von Amerika

World Health Organization - Weltgesundheitsorganisation

Zevalin, Carmustin (BCNU®), Etoposid, Cytarabinosid (Alexan®), Melphalan 


\subsection{Formelzeichen}

Deziliter $\quad \mathrm{dl}$

Grad Celsius $\quad{ }^{\circ} \mathrm{C}$

Gramm g

Kilogramm $\quad \mathrm{kg}$

Mega-Elektronenvolt $\mathrm{MeV}$

Mikroliter $\quad \mu l$

Millicurie $\quad \mathrm{mCi}$

Milligramm $\quad \mathrm{mg}$

Millimeter $\quad \mathrm{mm}$

Quadratmeter $\mathrm{m}^{2}$ 


\subsection{Elektronische Dokumentationsbögen}

Die in diesem Kapitel aufgeführten elektronischen Dokumentationsbögen stammen aus der Eingabeoberfläche der zentralen Datenbank des RIT-Netzwerkes, welche von dem unabhängigem Auftragsforschungsinstitut Alcedis $\mathrm{GmbH}$ verwaltet und beaufsichtigt wird.

Zugang zur Internetpräsenz des internationalen RIT-Registers (Stand 30.10.2011):

https://rde-med.alcedis.de/study-manager/riteu/

- Stammdaten

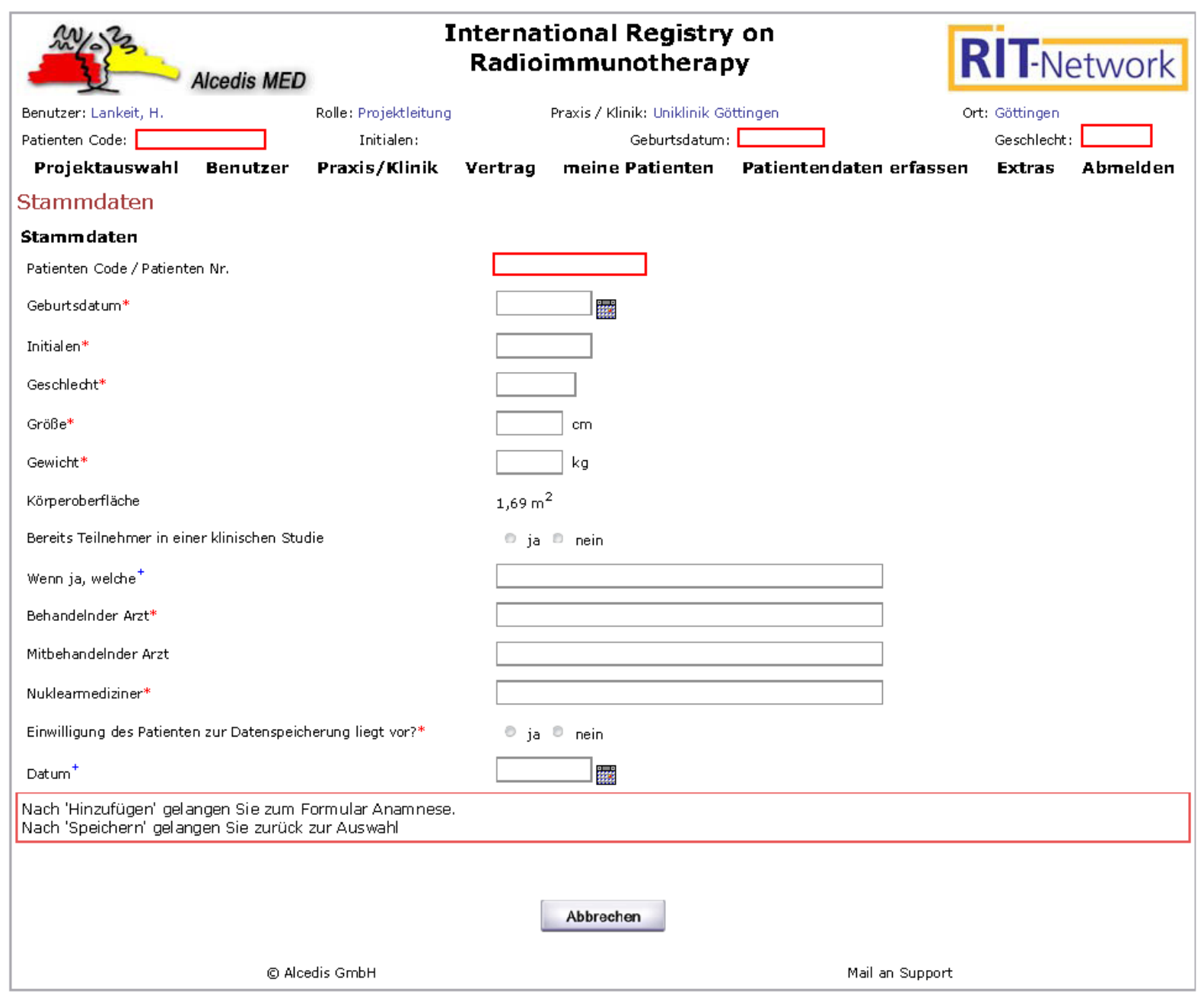




\section{- Anamnese}

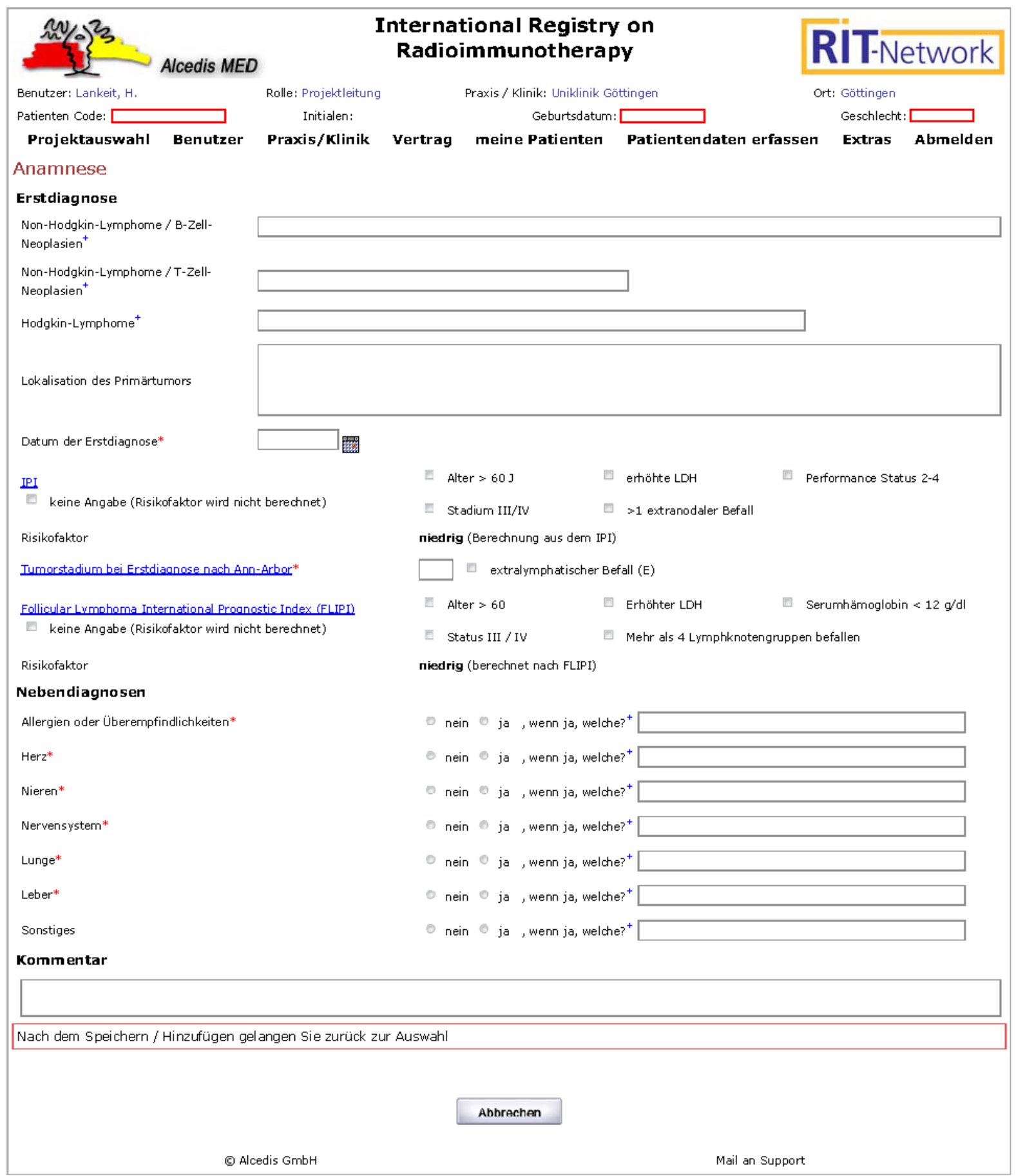


- Vortherapien

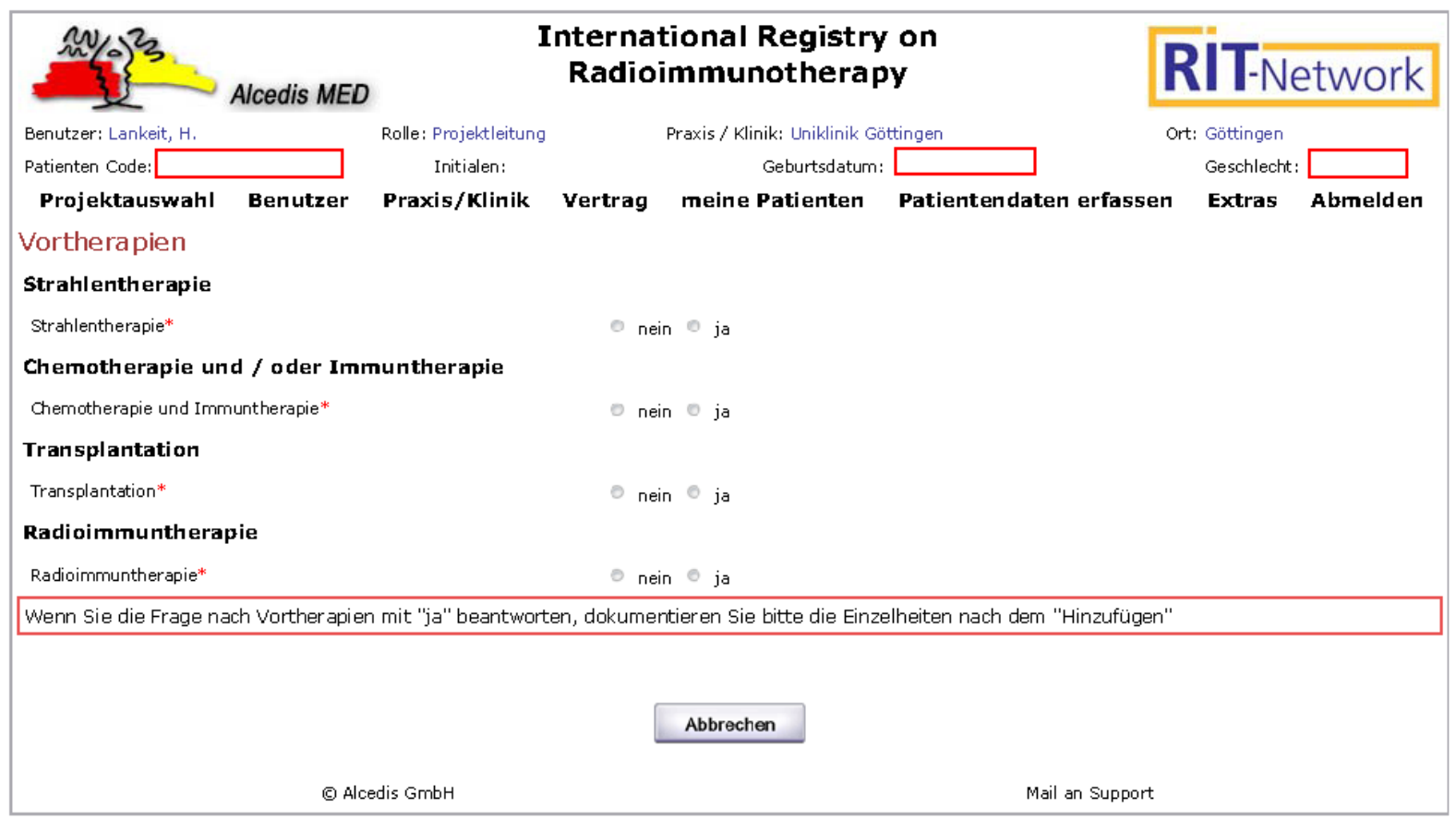


- Radioimmuntherapie

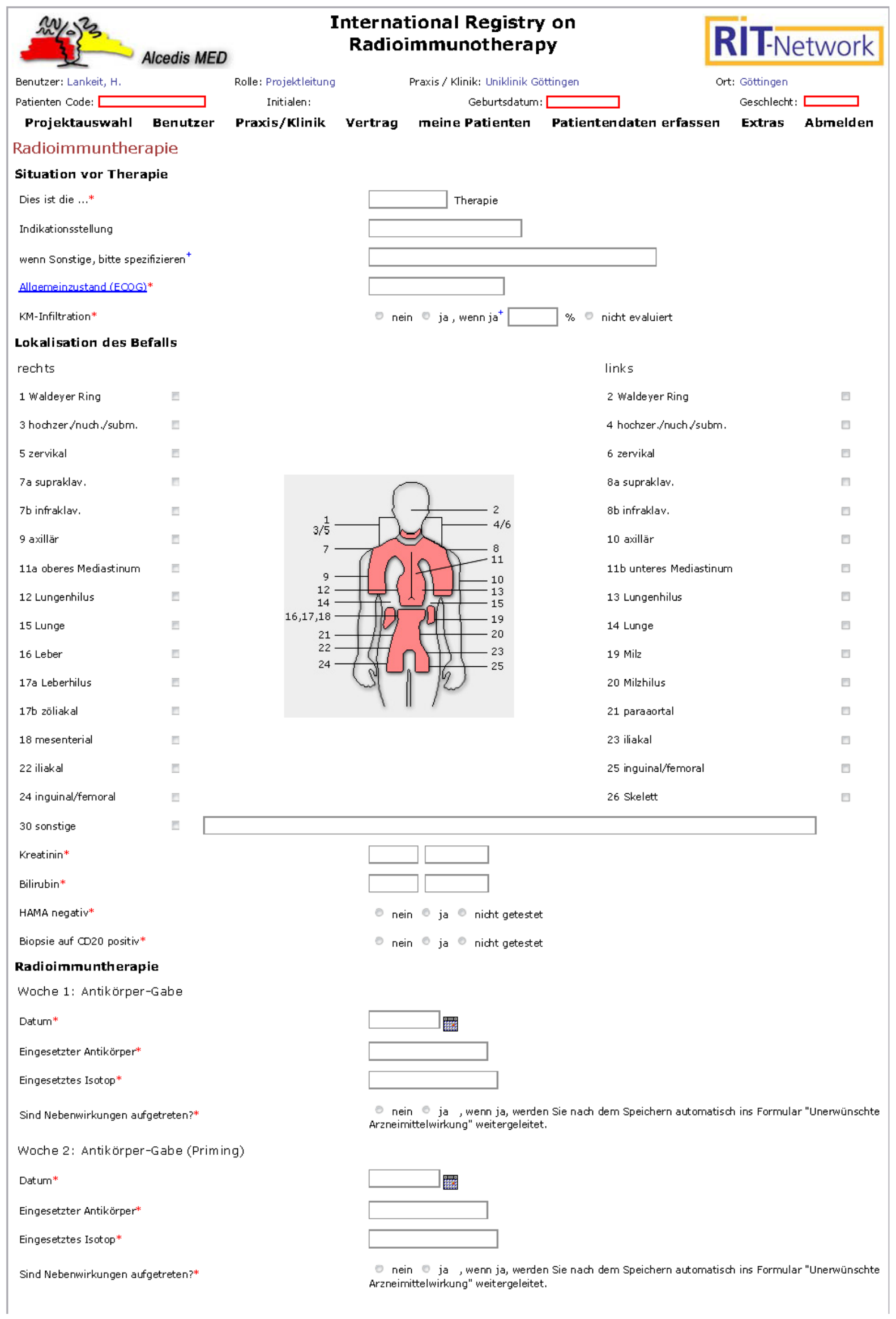




\section{Blutbilder}

Blutbild Therapiebeginn

Datum ${ }^{8}$

Hämoglobin $[\mathrm{g} / \mathrm{d}]^{+}$

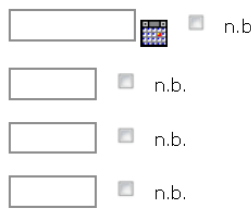

Thrombozyten [pro $\mu]^{+}$

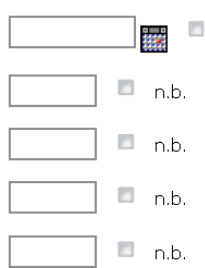

\section{Parameter}

Hämoglobin $[\mathrm{g} / \mathrm{dl}]^{\mathrm{g}}$

Leukozyten [pro nl]

Neutrophile Granulozyten [pro $\mu]^{\natural}$

Thrombozyten $[\text { pro } \mu]^{\alpha}$
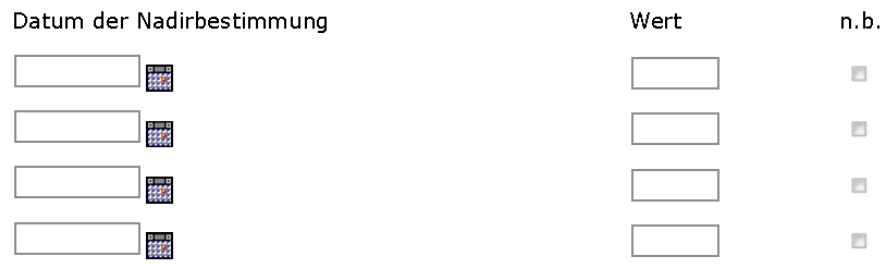

\section{Blutbilderholung}

Datum der kompletten Blutbilderholung ${ }^{\text {" }}$

Transfusion gegeben?

囯

\section{Transfusion}

Einheit(en)

Erythrozytenkonzentrat

Thrombozytenkonzentrat

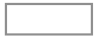

Wurden Medikamente zur Blutbilderholung eingesetzt?*

Medikament

Dosis / Einheit

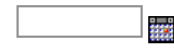

nein $\bigcirc j a$

Von

Bis
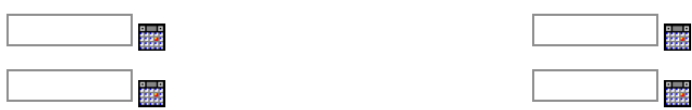

onein 0 ja

Von

Bis
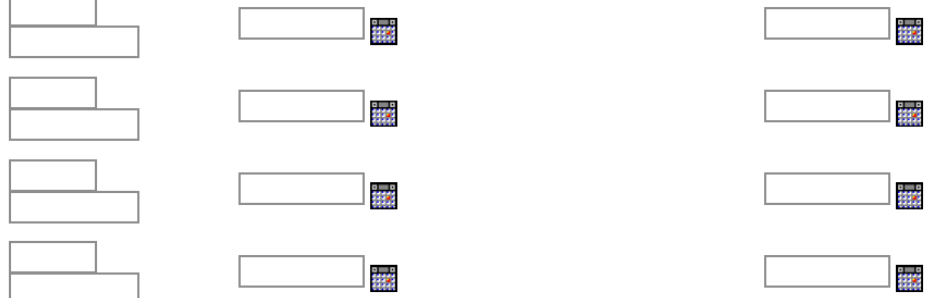

4

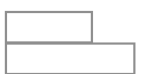

Sind Infektionen aufgetreten?

- nein ${ }^{\circ} \mathrm{ja}$, wenn ja, werden Sie nach dem Speichern automatisch ins Formular "Unerwünscht Arzneimittelwirkung" weitergeleitet.

Kommentar 
- Unerwünschte Arzneimittelwirkung

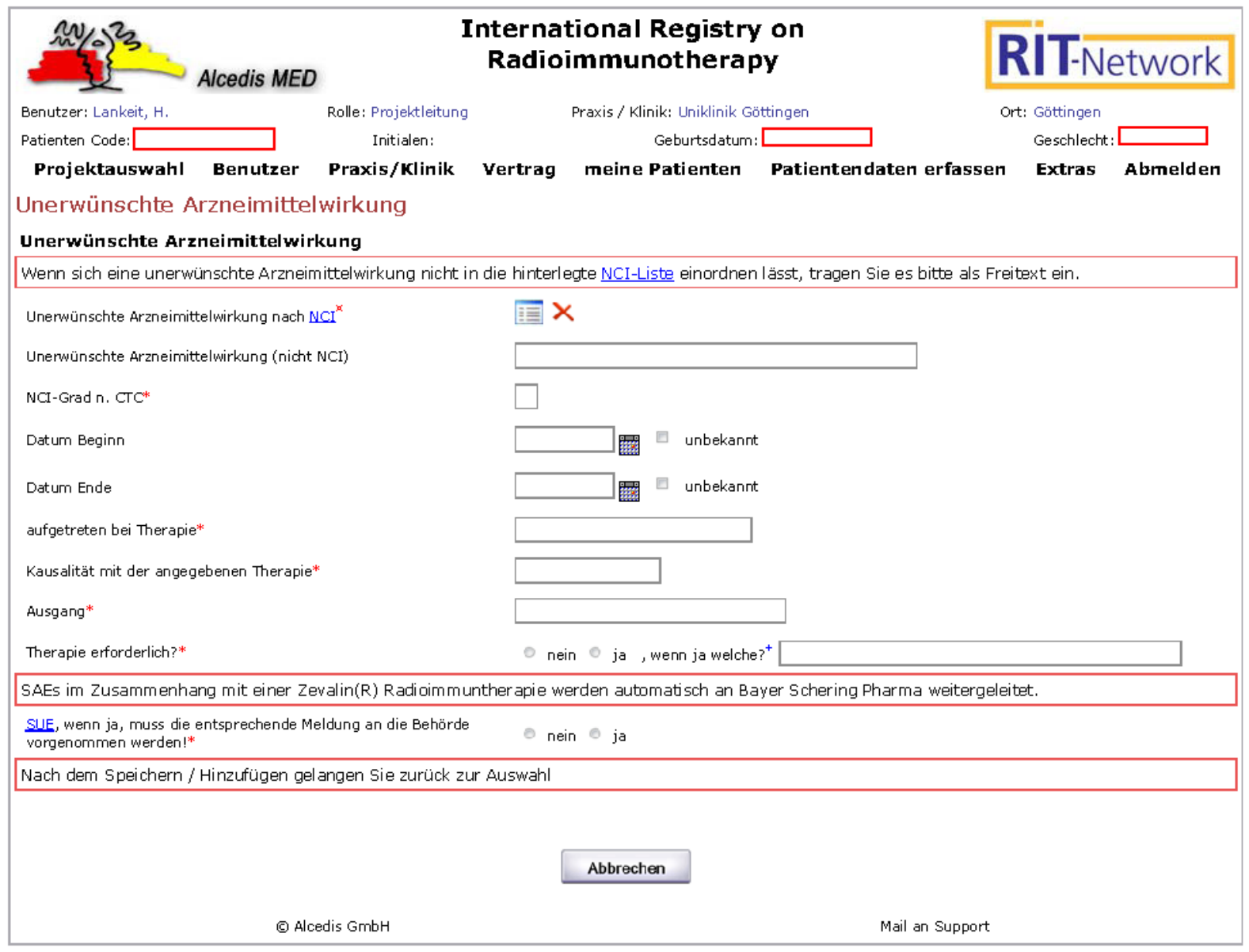




\section{- Schwerwiegende unerwünschte Arzneimittelwirkung}

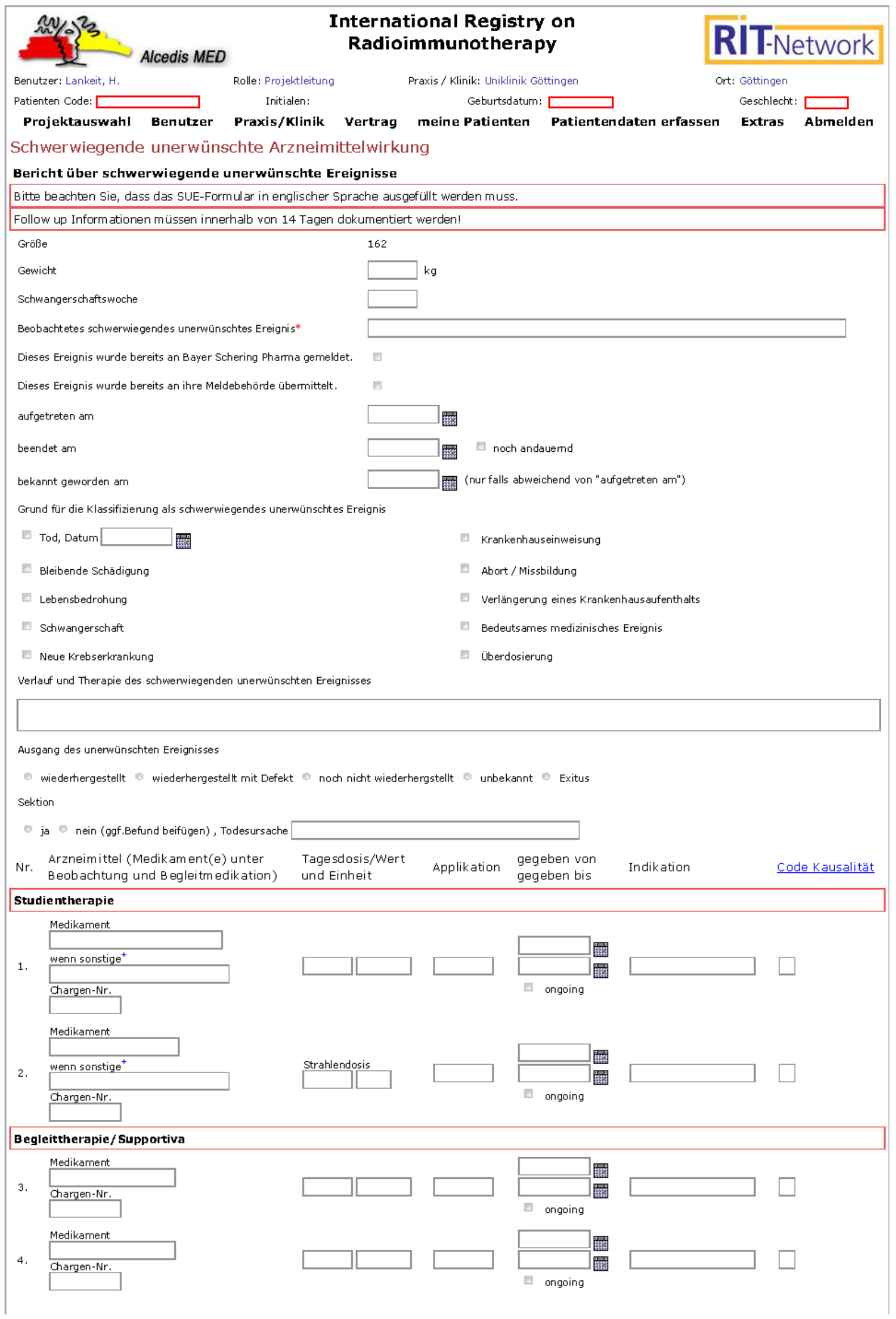




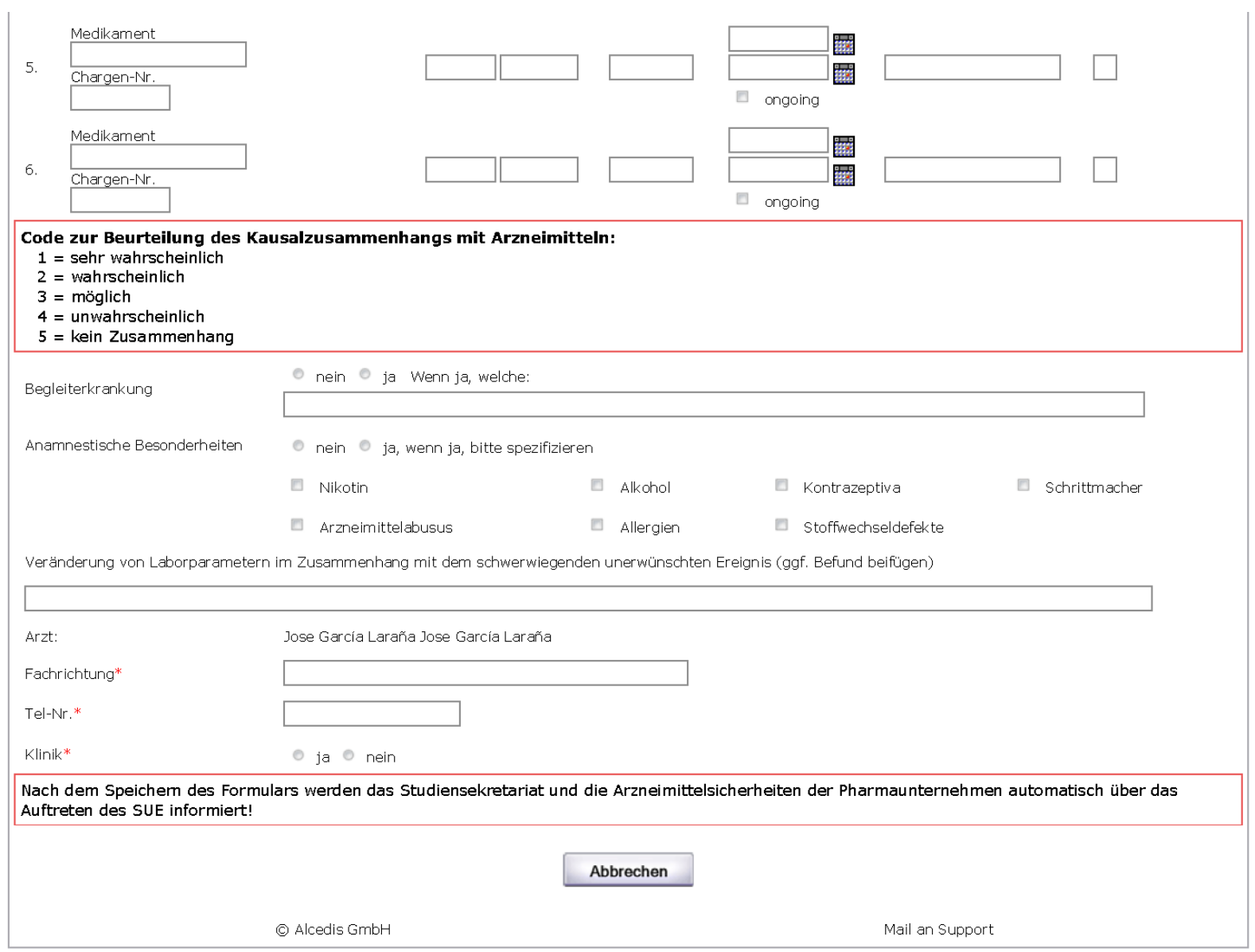




\section{- Verlaufsbeobachtungen}

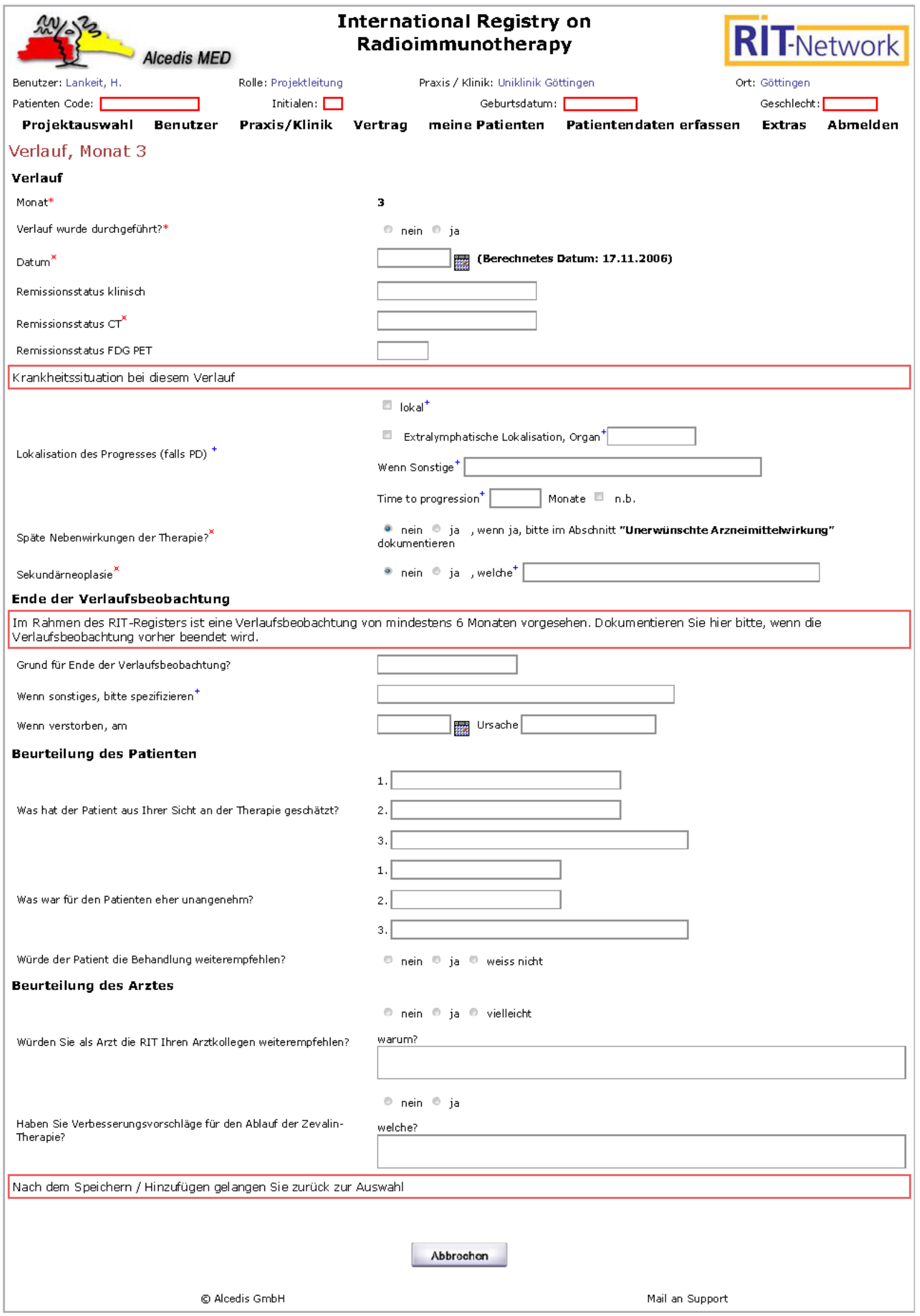


- Abschlussbeurteilung

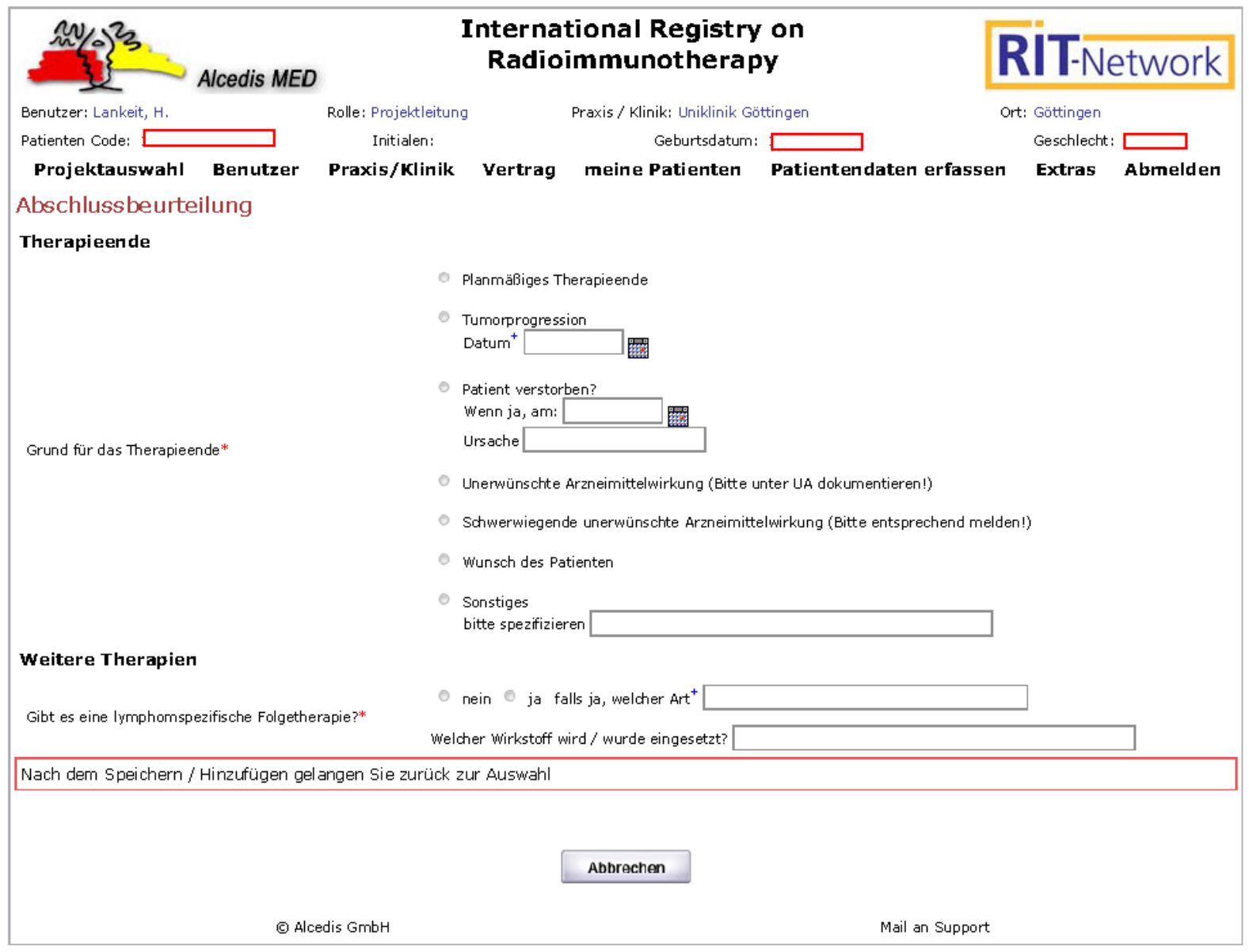

\section{- Unterschrift / Honorierung}

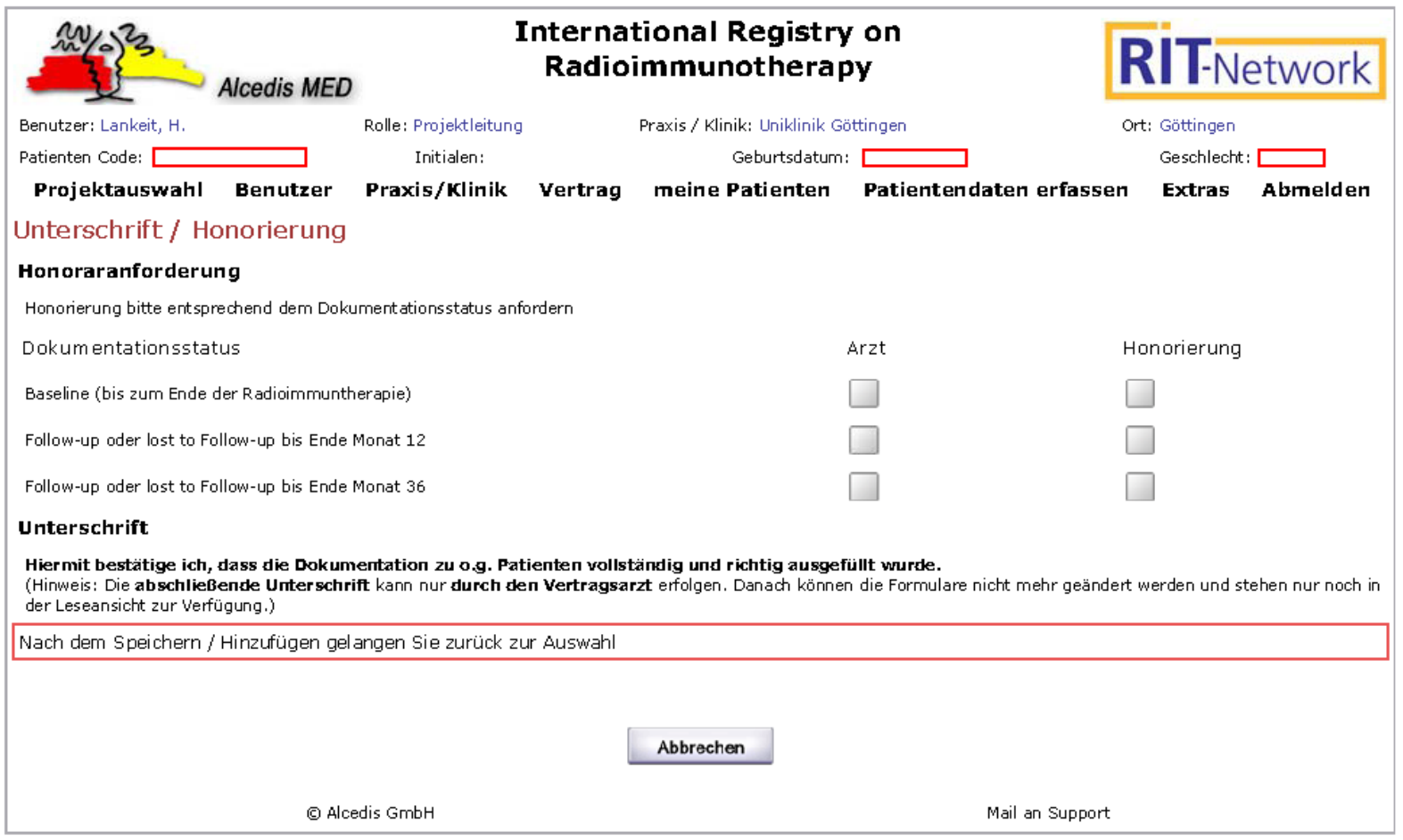




\section{Danksagung}

Ich möchte Herrn Prof. Dr. med. Lorenz Trümper ganz herzlich dafür danken, dass er mir als mein Doktorvater ermöglicht hat, in der von ihm geführten Abteilung Hämatologie und Onkologie der Universitätsmedizin Göttingen meine Dissertationsschrift anzufertigen.

Mein ganz besonderer Dank gilt Frau Dr. med. Karin Hohloch für ihre engagierte und freundliche Betreuung, umfangreiche Unterstützung und kompetente Hilfestellung im Rahmen meiner Dissertation.

Ganz herzlich bedanken möchte ich mich zudem bei der Firma Alcedis GmbH und insbesondere bei Frau Dr. Christine Windemuth-Kieselbach und Herrn Michael Lorsbach, die mir bei der Sichtung und Auswertung der Patientendaten eine große Hilfe waren.

Herrn Dr. Björn Hoxhold und Frau Imke Rösing danke ich von Herzen für ihre produktiven Ratschläge und qualifizierten Anmerkungen bei der Durchsicht meiner Dissertationsschrift. 


\section{Lebenslauf}

Am 24.04.1987 wurde ich, Henrike Katharina Lankeit, als drittes Kind meiner Eltern Michael und Katharina Lankeit, geb. Lindner, in Köln geboren. Ich habe eine Schwester (Mareike, geb. 16.04.1982) und einen Bruder (Frederik, geb 05.04.1985).

Nach unserem Umzug von Köln nach Göttingen besuchte ich von 1993 bis 1997 die Hainbundschule in Weende und nachfolgend von 1997 bis 1999 die Orientierungsstufe im Schulzentrum Göttingen-Nord. Von 1999 bis 2006 besuchte ich das Theodor-HeussGymnasium und erlangte dort am 30.06.2006 mit dem Abitur die allgemeine Hochschulreife (Note "sehr gut"). Das erste Halbjahr des 11. Schuljahres, also August 2003 bis Januar 2004, verbrachte ich in Buderim, Queensland, Australien und besuchte dort das Immanuel Lutheran College.

Nach meinem Abitur absolvierte ich mein dreimonatiges (November 2006 bis Februar 2007) Krankenpflegepraktikum in der Unfallchirurgischen Abteilung des Evangelischen Krankenhauses Göttingen Weende e.V., bevor ich im Sommersemester 2007 mein Studium der Humanmedizin an der Georg-August-Universität Göttingen begann. Im März 2009 bestand ich ebenda den 1. Abschnitt der Ärztlichen Prüfung mit der Note "gut". Im Verlauf meiner klinischen Ausbildung habe ich einen Monat (Juli bis August 2009) in der Abteilung Anästhesiologie und Intensivmedizin im Evangelischen Krankenhaus Köln Weyertal, einen Monat (Februar bis März 2010) in der Inneren Medizin im Klinikum Links der Weser gGmbH in Bremen, sowie jeweils zwei Wochen (im Juli 2010 und im September 2011) in der Klinik für Nieren- und Hochdruckkrankheiten des städtischen Klinikums Braunschweig gGmbH und in der Klinik für Frauenheilkunde und Geburtshilfe des Caritas-Krankenhauses St. Josef in Regensburg famuliert. Des Weiteren führte ich meine Famulatur in einer niedergelassenen Praxis im August 2011 in der gastroenterologischen Gemeinschaftspraxis im Facharztzentrum Regensburg bei Herrn Dr. Worlicek sowie in der kardiologischinternistischen Gemeinschaftspraxis im Facharztzentrum Regensburg bei Frau Dr. Laschinger-Oestreicher durch. 
Im Mai 2010 habe ich in der Abteilung Hämatologie und Onkologie im Zentrum Innere Medizin der Medizinischen Fakultät der Georg-August-Universität Göttingen unter der Leitung von Prof. Dr. med. Lorenz Trümper begonnen an der vorliegenden Dissertation zu arbeiten. Während dieser Zeit wurde ich von Frau Dr. med. Karin Hohloch betreut.

Voraussichtlich werde ich im Sommersemester 2012 mein Praktisches Jahr in der Frauenklinik des Universitätsspitals Bern, Schweiz (Wahlfach) beginnen und darauf folgend in der Inneren Medizin des DIAKO Krankenhauses in Bremen sowie in der chirurgischen Abteilung des Evangelischen Krankenhauses Göttingen Weende e.V. ableisten. Aller Voraussicht nach werde ich zwischen April und Juni 2013 den 2. Abschnitt der Ärztlichen Prüfung beschreiten. 